\title{
Psychostimulant Use Disorder, an Unmet Therapeutic Goal: Can Modafinil Narrow the Gap?
}

\author{
Melinda Hersey ${ }^{1 t}$, Amanda K. Bacon ${ }^{1 t}$, Lydia G. Bailey ${ }^{1 t}$, Mark A. Coggiano", \\ Amy H. Newman ${ }^{1}$, Lorenzo Leggio ${ }^{1,2,3}$ and Gianluigi Tanda ${ }^{1 *}$
}

\begin{abstract}
${ }^{1}$ Medication Development Program, Molecular Targets and Medication Discovery Branch, National Institute on Drug Abuse, Intramural Research Program, National Institutes of Health, Baltimore, MD, United States, ${ }^{2}$ Clinical Psychoneuroendocrinology and Neuropsychopharmacology Section, Translational Addiction Medicine Branch, National Institute on Drug Abuse Intramural Research Program, National Institutes of Health, Baltimore, MD, United States, ${ }^{3}$ National Institute on Alcohol Abuse and Alcoholism, Division of Intramural Clinical and Biological Research, National Institutes of Health, Bethesda, MD, United States
\end{abstract}

\section{OPEN ACCESS}

Edited by: Anna R. Carta,

University of Cagliari, Italy

Reviewed by:

Thomas M. Keck,

Rowan University, United States Marco Bortolato,

The University of Utah, United States

*Correspondence:

Gianluigi Tanda

gtanda@intra.nida.nih.gov

tThese authors have contributed equally to this work and share first

authorship

Specialty section:

This article was submitted to Neuropharmacology,

a section of the journal

Frontiers in Neuroscience

Received: 20 January 2021

Accepted: 20 April 2021

Published: 26 May 2021

Citation:

Hersey M, Bacon AK, Bailey LG, Coggiano MA, Newman AH, Leggio L and Tanda G (2021) Psychostimulant Use Disorder, an Unmet Therapeutic Goal: Can Modafinil Narrow the Gap?

Front. Neurosci. 15:656475

doi: 10.3389/fnins.2021.656475
The number of individuals affected by psychostimulant use disorder (PSUD) has increased rapidly over the last few decades resulting in economic, emotional, and physical burdens on our society. Further compounding this issue is the current lack of clinically approved medications to treat this disorder. The dopamine transporter (DAT) is a common target of psychostimulant actions related to their use and dependence, and the recent availability of atypical DAT inhibitors as a potential therapeutic option has garnered popularity in this research field. Modafinil (MOD), which is approved for clinical use for the treatment of narcolepsy and sleep disorders, blocks DAT just like commonly abused psychostimulants. However, preclinical and clinical studies have shown that it lacks the addictive properties (in both behavioral and neurochemical studies) associated with other abused DAT inhibitors. Clinical availability of MOD has facilitated its offlabel use for several psychiatric disorders related to alteration of brain dopamine (DA) systems, including PSUD. In this review, we highlight clinical and preclinical research on MOD and its R-enantiomer, R-MOD, as potential medications for PSUD. Given the complexity of PSUD, we have also reported the effects of MOD on psychostimulantinduced appearance of several symptoms that could intensify the severity of the disease (i.e., sleep disorders and impairment of cognitive functions), besides the potential therapeutic effects of MOD on PSUD.

\footnotetext{
Keywords: cocaine, modafinil, dopamine, dopamine transporter blocker, psychostimulant use disorder, drug abuse and dependence, drug addiction
}

Abbreviations: ADHD, attention deficit hyperactivity disorder; CNS, central nervous system; CPP, conditioned place preference; CBT, cognitive behavioral therapy; DA, dopamine; DAT, dopamine transporter; DS, dorsal striatum; ICSS, intracranial self-stimulation; MOD, modafinil; METH, methamphetamine; NAcc, nucleus accumbens; NAS, nucleus accumbens shell; NE, norepinephrine; NET, norepinephrine transporter; PET, positron emission tomography; PFC, prefrontal cortex; PSUD, psychostimulant use disorder; R-MOD, (R)-enantiomer of modafinil; VMAT2, vesicular monoamine transporter; VS, ventral striatum. 


\section{INTRODUCTION}

Psychostimulant use disorder is a complex disease defined by DSM-5 which includes both former (DSM-IV) diagnoses of abuse and dependence on a psychostimulant, such as cocaine or amphetamines. While illicit drugs have long been a societal concern, drug use rates have been growing in recent years. Globally, stimulants such as cocaine and amphetamines are used by approximately $0.35-0.4 \%$ and $0.7-0.77 \%$ of the population, respectively (Peacock et al., 2018; Farrell et al., 2019). Of these subpopulations, $16 \%$ are dependent on cocaine, while $11 \%$ are dependent on amphetamines (Farrell et al., 2019). In the United States, it was estimated that about 5.5 million people age 12 and older used cocaine in 2018 ( $2 \%$ of the United States population) (SAMHSA, 2018) and 1.9 million people age 12 and older used METH in 2018 ( $0.7 \%$ of the United States population) (SAMHSA, 2018). A major issue with substance use disorders is the risk of overdose. Recent data show that between 2012 and 2018, drug overdoses involving cocaine more than tripled, and drug overdoses involving abused psychostimulants increased nearly five-fold (Hedegaard et al., 2020).

Classically, the neurobiology underlying PSUD has focused on the neurotransmitter dopamine (DA) for its role in reward processing (Wise and Rompre, 1989; Wise, 2008; Arias-Carrión et al., 2010; Taber et al., 2012). Indeed, commonly abused stimulants exert effects on brain DA levels through their interactions with the neuronal membrane DAT (Das, 1993; Nestler, 2005). Increased DA levels after psychostimulant administration lead to arousal and euphoria, which facilitate the transition from the initial recreational use to continued excessive use, and parallel the potential clinical development of addiction in patients with the most severe form of the disorder (Compton et al., 2018).

The clinical severity of PSUD can be often worsened by medical and mental health comorbidities, e.g., mood and sleep disorders (Mahfoud et al., 2009; Gould, 2010; Torrens and Rossi, 2015). Furthermore, PSUD may be associated with cognitive impairment, which in turn lead to higher treatment dropout rates (Sofuoglu et al., 2013, 2016; Nuijten et al., 2016). These indicate a potential treatment avenue to ameliorate some of the effects of PSUD, which may contribute to increased abstinence rates overall.

Treatment of PSUD relies primarily on behavioral remedies, which may include 12-step facilitation, contingency management, relapse prevention, motivational enhancement therapy, and CBT (for a review, see: Vocci and Montoya, 2009). However, these approaches are time- and resourceintensive and their effect sizes are sub-optimal: integration with effective pharmacotherapies would be likely to improve outcomes and success rates. However, to date there are no approved pharmacologic treatments for PSUD (Phillips et al., 2014). Medications such as antidepressants, DA agonists/partial agonists, mood stabilizers, neuro-protectives, and agonist-like replacement therapy (de Lima et al., 2003; Elkashef et al., 2005; Diana, 2011; Phillips et al., 2014; Jordan et al., 2019) have all been tested with minimal success. The lack of pharmacological treatments for PSUD is a driving force for research toward the development of novel medications.

Among the potential pharmacotherapeutic options for PSUD is MOD, a clinically available medication that inhibits the uptake of DA by blocking DAT (Mignot et al., 1994; Loland et al., 2012). This pharmacological effect is shared with abused psychostimulants but, in spite of that, MOD shows behavioral and neurochemical actions that suggest limited, if any, potential for misuse (Jasinski, 2000; Deroche-Gamonet et al., 2002; Myrick et al., 2004; Food and Drug Administration, 2007; Vosburg et al., 2010; Mereu et al., 2020). Currently, this agent is prescribed for its wake-promoting effects (Czeisler et al., 2005; Kumar, 2008), consistent with its approval for narcolepsy, shift work sleep disorder, and obstructive sleep apnea/hypopnea syndrome. Off-label, MOD has been used for its pro-cognitive effects, especially in patients with cognitive impairment associated with psychiatric disorders (Peñaloza et al., 2013; Turner et al., 2014). During the last two decades, MOD has been tested as a potential medication to treat some of the primary (dependence) and secondary (cognitive and sleep disorders) symptoms of PSUD, representing a potential additional treatment option for selected populations affected by PSUD.

In this review, we will mainly focus on preclinical studies showing how MOD and R-MOD (its R-enantiomer) interact with DAT, the DAergic system, and the reinforcing actions of abused psychostimulants. We will also review clinical studies where MOD efficacy as a potential treatment for PSUD has been evaluated, including for related symptoms such as alteration of sleep and cognitive dysfunction.

\section{MODAFINIL PHARMACOLOGY RELATED TO PSUD}

Modafinil [2-(Diphenylmethyl) sulfinyl acetamide; Alertec, Modavigil, Provigil] and its long-acting, enantiopure form, R-MOD (Nuvigil, Artvigil) (Wong et al., 1999), are clinically available and prescribed as wake-promoting agents for narcolepsy and sleep disorders (Bastoji and Jouvet, 1988; Broughton et al., 1997; US Modafinil in Narcolepsy Multicenter Study Group, 1998, 2000). Early evidence suggested that MOD had a weak, low $\mu \mathrm{M}$ affinity, but relatively good selectivity, for DAT (Mignot et al., 1994), confirmed by more recent studies (Madras et al., 2006; Loland et al., 2012). Thus, the main mechanism of action for MOD appears predominantly driven by actions at neural membrane DATs to stimulate catecholamine neurotransmission (Wisor et al., 2001; Madras et al., 2006). DAT knockout mice were used to confirm the importance of DAT in the mechanism of action of MOD, as studies have found that the pharmacological wake-promoting effects of MOD administration were abolished in those mutant mice (Wisor et al., 2001). Volkow et al. (2009) used PET to show that, after oral administration, MOD (200 to $400 \mathrm{mg}$ ) occupies and blocks DAT in the human brain (caudate, NAcc, and putamen). The latter effect was also shown for the enantiomer, R-MOD (Spencer et al., 2010). Further, as a result of the DAT inhibition induced by administration of MOD or $\mathrm{R}-\mathrm{MOD}$, increased brain DA levels can be observed in several 
dopaminergic nerve terminal regions (Ferraro et al., 1996c; Wisor et al., 2001; Volkow et al., 2009; Loland et al., 2012). Further, DAT trafficking could be affected by psychostimulants. Administration of DAT substrates like METH and amphetamine decreases the trafficking of DAT to the cell surface (Saunders et al., 2000; Zahniser and Sorkin, 2009), while DAT inhibitors like cocaine have been shown to increase DAT trafficking to the cell surface (Daws et al., 2002; Little et al., 2002; Zahniser and Sorkin, 2009). Although the effects of MOD administration on DAT trafficking have yet to be fully elucidated, it has been shown that MOD prevents METH-induced decreases in DAT immunoreactivity 6 days after treatment (Raineri et al., 2012).

Beyond DAT, MOD does not show significant affinity for other important pharmacological brain targets. For example, MOD affinity for the NET falls in the $100 \mu \mathrm{M}$ range (Madras et al., 2006), and it is still unclear if the increases in brain NE levels induced by MOD are the result of its interaction with NET (see for review Mereu et al., 2013). These effects on brain NE levels in PFC and rostro-medial hypothalamus (de Saint Hilaire et al., 2001) could be of interest due to a welldocumented role for NE in wakefulness and arousal (reviewed in Mitchell and Weinshenker, 2010). Interestingly, MOD did not show direct activity on trace amine-associated receptor 1 (TAAR1) (Madras et al., 2006), in contrast to amphetamines (Xie and Miller, 2009; Liu et al., 2020). MOD has been shown to have indirect actions on TAAR1 through activation of DAT, which can augment TAAR1 activation (Madras et al., 2006). TAAR1 has been implicated in wakefulness, which represents a predictable effect given the receptor's ability to modulate the activity of other monoamine systems (Revel et al., 2013; Liu et al., 2020). In a recent report, deletion of TAAR1 receptor in mice did not produce substantial effects on MOD-induced wakefulness as compared to WT mice (Schwartz et al., 2018). In the same report, reductions in MOD-induced gamma-band activity in EEG studies in TAAR1 KO mice were found, and the authors suggest that TAAR1 may regulate neurophysiological factors related cortical and cognitive functions (Schwartz et al., 2018).

Regardless of its affinity for pharmacological targets, MOD has been reported to affect the levels of several neurotransmitters. MOD stimulates brain glutamate levels in the hypothalamus (medial preoptic area and posterior hypothalamus), thalamus (ventromedial and ventrolateral regions), and hippocampus (Ferraro et al., 1997b, 1999), and it has been shown to decrease the levels of GABA in the NAcc, hypothalamus (medial preoptic area and posterior hypothalamus), striatal, and pallidal regions (Ferraro et al., 1996b, 1997a, 1999). MOD induced stimulation in brain serotonin levels in the PFC (Ferraro et al., 2000; de Saint Hilaire et al., 2001), increases in histamine levels and/or activation in the tuberomammillary nucleus and the anterior hypothalamus (Scammell et al., 2000; Ishizuka et al., 2003, 2008), and limited activation of orexin/hypocretin neurons in the perifornical areas and lateral hypothalamus (Chemelli et al., 1999; Scammell et al., 2000; Willie et al., 2005) has also been observed (reviewed in Kumar, 2008; Minzenberg and Carter, 2008; Mereu et al., 2013).

In addition to its effects on neurotransmitter levels, MOD administration affects the induction and inhibition of hepatic cytochrome P450 isoenzymes (Robertson et al., 2000). In vitro, MOD competitively inhibits CYP2C19 and suppresses CYP2C9, as well as moderately induces CYP1A2, CYP3A4, and CYP2B6 (Robertson et al., 2000). Pharmacokinetic studies in vivo with warfarin and ethinylestradiol, which react with CYP2C9 and CYP3A4 respectively, have not shown the same magnitude of effect as in vitro studies (Robertson and Hellriegel, 2003). Through MOD's induction and inhibition of the $P 450$ isoenzymes, MOD co-administration may decrease or prolong plasma concentrations of other drugs metabolized through these enzymes (Schwartz, 2005). There have been clinical reports of MOD interactions with medications, for example, cyclosporine and clomipramine. Specifically, the immunosuppressive effect of cyclosporine decreased after $200 \mathrm{mg} /$ day MOD, which appeared to be from CYP3A4 induction (for a review, see e.g., Robertson and Hellriegel, 2003). A patient treated with clomipramine was found to lack functional CYP2D6, and the ancillary CYP2C19 pathways inhibited by MOD contributed to increased clomipramine levels in the blood (Robertson and Hellriegel, 2003). MOD also has notable effects as a facilitator of electrotonic coupling in neurons and astroglia through actions at gap junctions (Garcia-Rill et al., 2007; Urbano et al., 2007; Liu et al., 2013; Duchêne et al., 2016; Mereu et al., 2020). In particular, it has been shown that the gap junction inhibitor carbenoxolone blunted the ability of MOD to potentiate self-administration of cocaine in rats (Mereu et al., 2020). These properties are likely important for the agent's pharmacological actions, as well as interactions with other drugs and biomolecules.

\section{Modafinil, DAT Inhibition, and Potential Abuse Liability}

As a result of inhibition of DAT, it is not surprising that MOD activities could overlap with some of those observed after administration of commonly abused psychostimulants. However, as reported in Table 1, some of its actions seem directed to improve specific symptoms observed in patients with a PSUD diagnosis, i.e., impairments in cognition, sleep, cardiovascular function, and mood disturbances, as well as elevated neuroinflammation. Moreover, MOD fails to display the abuse potential (Jasinski, 2000; Deroche-Gamonet et al., 2002; Myrick et al., 2004; Food and Drug Administration, 2007; Vosburg et al., 2010) or the withdrawal symptoms (Hermant et al., 1991; Myrick et al., 2004) observed with typical psychostimulants. Indeed, to our knowledge, only a very few anecdotical reports of MOD abuse and dependence have been reported in the literature (Kate et al., 2012; Ozturk and Deveci, 2014; Krishnan and Chary, 2015) despite the climbing rates of its non-medical use as a cognitive enhancer in schools and at the workplace (Sharif et al., 2021). Further, important behavioral and neurochemical differences between MOD, or R-MOD, and typical abused psychostimulants have been found in preclinical studies, suggesting they have a unique pharmacological, psychostimulant profile. Taken together, these actions highlight the potential for MOD to reduce the harm associated with the complexity of the symptoms in PSUD. 
TABLE 1 | Symptoms related to PSUD and potential therapeutic actions of MOD.

\begin{tabular}{|c|c|c|c|}
\hline \multicolumn{2}{|c|}{ PSUD symptoms } & \multicolumn{2}{|c|}{ Actions of MOD } \\
\hline \multirow[t]{5}{*}{$\begin{array}{l}\text { Recreational use, misuse, and } \\
\text { potential for dependence (DEA } \\
\text { schedule } 1 \text { or 2) }\end{array}$} & Gawin (1991); Barr et al. (2006) & Low abuse liability (DEA schedule 4) & $\begin{array}{l}\text { Jasinski (2000); Deroche-Gamonet } \\
\text { et al. (2002); Myrick et al. (2004); Food } \\
\text { and Drug Administration (2007); } \\
\text { Vosburg et al. (2010) }\end{array}$ \\
\hline & & Substitute for psychostimulants & $\begin{array}{l}\text { Gold and Balster (1996); Reichel and } \\
\text { See (2012) }\end{array}$ \\
\hline & & Decreased cocaine use & Dackis et al. (2005); Hart et al. (2008) \\
\hline & & Decreased nicotine use & Wang et al. (2015) \\
\hline & & Decreased METH use & $\begin{array}{l}\text { Shearer et al. (2009); De La Garza et al. } \\
\text { (2010) }\end{array}$ \\
\hline Cardiovascular issues & $\begin{array}{l}\text { Lange and Hillis (2001); Kaye et al. } \\
\text { (2007); Duflou (2020) }\end{array}$ & $\begin{array}{l}\text { Regulates heart rate disruptions } \\
\text { associated with amphetamines }\end{array}$ & $\begin{array}{l}\text { Makris et al. (2004); De La Garza et al. } \\
\text { (2010) }\end{array}$ \\
\hline Impaired cognition & Bolla et al. (1999); Nordahl et al. (2003) & $\begin{array}{l}\text { Improved attention, concentration, } \\
\text { executive function }\end{array}$ & $\begin{array}{l}\text { Pigeau et al. (1995); Minzenberg and } \\
\text { Carter (2008); Killgore et al. (2009); } \\
\text { Finke et al. (2010); Dean et al. (2011) }\end{array}$ \\
\hline \multirow[t]{3}{*}{ Impaired mood } & $\begin{array}{l}\text { Rounsaville et al. (1991); Salo et al. } \\
\text { (2011) }\end{array}$ & Improved mood & Pigeau et al. (1995) \\
\hline & & Improve depressive illness & Frye et al. (2007) \\
\hline & & Increased/decreased anxiety & Sarnyai et al. (1995); Salo et al. (2011) \\
\hline Elevated neuroinflammation & Kousik et al. (2012) & $\begin{array}{l}\text { Protects against PSUD-related } \\
\text { neuroinflammation }\end{array}$ & Raineri et al. (2012) \\
\hline \multirow[t]{2}{*}{ Sleep disruptions } & $\begin{array}{l}\text { Schierenbeck et al. (2008); Hasler et al. } \\
\text { (2012) }\end{array}$ & Wake-promoting agent & $\begin{array}{l}\text { Beusterien et al. (1999); Scammell et al. } \\
\text { (2000) }\end{array}$ \\
\hline & & Fatigue reducing & Pigeau et al. (1995) \\
\hline
\end{tabular}

$\overline{M E T H}=$ methamphetamine; DEA = Drug Enforcement Agency; PSUD = psychostimulant use disorder; MOD = modafinil.

In the next sections, we will briefly highlight some of the pharmacological actions of MOD, i.e., increased wakefulness, improved cognition and cardiovascular function, that could play a potential role in its therapeutic activity against PSUD (summarized in Table 1).

\section{Modafinil Interactions With Sleep and Wakefulness Activities}

Modafinil was introduced as a wake-promoting agent in the 1990s and approved by the US FDA to treat excessive sleeping (narcolepsy, shift work sleep disorder, obstructive sleep apnea/hypopnea syndrome) (Bastoji and Jouvet, 1988; Broughton et al., 1997; US Modafinil in Narcolepsy Multicenter Study Group, 1998, 2000). In addition to its approved uses, MOD is often used off-label for the treatment of fatigue symptoms in neurological, psychiatric, and excessive fatigue disorders (reviewed in Kumar, 2008). Like amphetamines, the molecular mechanism by which MOD imposes wakefulness has been largely debated. The mechanism is suspected to be linked to the inhibition of monoamine transport by plasma membrane transporters like DAT. In particular, studies have shown that DAT and DA regulation in key areas involved in wakefulness/sleep (i.e., medial preoptic area and posterior hypothalamus) is crucial in the wake-promoting effects of amphetamines and MOD (Jones et al., 1977; Nishino and Mignot, 1997; Nishino et al., 1998; Wisor et al., 2001). Further, researchers have shown that DA activity fluctuates with arousal state (Trulson, 1985).
MOD's effects on other neurotransmitters are also suspected to play a role in the wakefulness-promoting properties of this agent (Boutrel and Koob, 2004). For example, changes in brain NE neurotransmission have been suggested to play a role in wakefulness and arousal (reviewed in Mitchell and Weinshenker, 2010), and MOD stimulation of brain NE levels in PFC and rostro-medial hypothalamus (de Saint Hilaire et al., 2001) could be related to its effect on sleep disturbances. While it is still unclear if NET blockade is the mechanism of action related to MOD stimulation of brain NE levels, some studies have suggested that NET inhibition is less efficacious in the promotion of wakefulness than DAT inhibition (Nishino and Mignot, 1997; Nishino et al., 1998). It is of interest to note that MOD, as highlighted above, interacts with other key neurotransmitters and systems that could play a role in the regulation of sleep, including glutamate, GABA, serotonin, histamine, and orexin/hypocretin (reviewed in Monti, 2013). There is also evidence that MOD increases glutamine synthetase in the rat brain, an enzyme that converts glutamate to glutamine for storage, which may be important for the wakefulness effects of MOD (Touret et al., 1994). The orexin system has a well-established role in sleepwake regulation (Espana et al., 2001; Sakurai, 2007). MOD administration increases the expression of c-Fos (a marker of neuronal activation) in orexin neurons in the hypothalamus (Chemelli et al., 1999; Scammell et al., 2000). Orexin neuronal projections can activate histamine release in the hypothalamus as well (Huang et al., 2001; Ishizuka et al., 2002). Histamine also has a well-documented role in regulating sleep-wake cycle 
(Haas et al., 2008). Interestingly, MOD administration produced activation of histaminergic cells (Scammell et al., 2000) but only in the presence of an intact orexin system (Ishizuka et al., 2010). Further, decreases in histamine or loss of histamine neurons blunted MOD-induced increases in locomotion (Ishizuka et al., 2008) as well as the drug's wake-promoting actions (Yu et al., 2019). On the other hand, orexin-null mice displayed heightened wakefulness following MOD administration compared to wildtype mice (Willie et al., 2005). These findings suggest impaired regulation of the arousal system following removal of orexin, but they also suggest that orexin is not necessarily required for MOD's wake-promoting actions, or that in those mice possible neuronal adaptations would substitute for removal of orexin.

\section{Modafinil Interactions With Cognitive Functions}

Enhancements in cognitive functions have been reported following MOD administration in rodents and humans (Turner et al., 2003; Minzenberg and Carter, 2008; Cope et al., 2017). MOD produces dose-dependent improvements in working memory (Béracochéa et al., 2001; Ward et al., 2004; Piérard et al., 2006), speed of learning (Béracochéa et al., 2002, 2003; Ward et al., 2004), and sustained attention (Morgan et al., 2007) in animals. In humans, MOD produces similar effects, improving memory and attention (reviewed in Minzenberg and Carter, 2008). Importantly, MOD heightens attention independently of its effects on wakefulness/arousal (Cope et al., 2017). MOD administration elicits changes in activation of brain regions associated with cognition, including the hippocampus (Ferraro et al., 1997b; Shuman et al., 2009; Brandt et al., 2014; Yan et al., 2015) and the PFC (Müller et al., 2004; González et al., 2014, 2018). Thus, improvements in cognitive functions associated with MOD's actions on the dopaminergic system may underlie those specific changes in DA transmission (reviewed in Minzenberg and Carter, 2008; Mereu et al., 2013), for example in the PFC, that have been recognized for their role in working memory (Sawaguchi and Goldman-Rakic, 1991, 1994). Further, DA receptors can be found on glutamatergic pyramidal cells (Tseng and O'Donnell, 2004) and GABAergic neurons (Tseng and O'Donnell, 2007) in the PFC where they can gate glutamatergic and GABAergic transmission linked to cognition (reviewed in Minzenberg and Carter, 2008). MOD's effects on brain NE may also affect cognition due to NE's established role in modulation of cognitive function (reviewed in Chamberlain and Robbins, 2013). Stimulation of NE neurotransmission following MOD administration is also implicated in cognition (Minzenberg and Carter, 2008), while MOD actions on the acetylcholine system have been shown to have effects on learning and memory (reviewed in Mereu et al., 2013). It has also been shown that MOD produced increased motivation, likely by activating D1 receptors (Young and Geyer, 2010). Importantly, MOD is an appealing candidate to target cognitive dysfunction associated with ADHD and psychiatric disorders (Ballon and Feifel, 2006), as well as PSUD. Specifically, treatment with MOD has been shown to improve cognition in PSUD patients (Dean et al., 2011) (see also the "Human studies" sections below).
The pro-cognitive effects of MOD have stimulated a debate about an ethical dilemma and potential concern regarding its rapidly increasing off-label, non-medical use in healthy individuals to improve attention, focus, memory, and cognitive functions (Cakic, 2009; Sahakian and Morein-Zamir, 2011; Peñaloza et al., 2013).

\section{Modafinil/DAT Inhibition and Inflammation}

Additional potential actions of MOD include the ability to act as an anti-inflammatory agent. Specifically, MOD has been shown to reduce neuroinflammation via suppressing inflammatory cytokines (Han et al., 2018), T-cell differentiation (Brandao et al., 2019), monocyte recruitment/activation (Zager et al., 2018), and activation of glial cells (Raineri et al., 2012). This MOD-induced immune activation may be essential for decreasing the neurotoxic and inflammatory consequences of many diseases including PSUD, an exceptionally important effect given that many stimulants are pro-inflammatory in nature. METH administration is marked by increases in TNF- $\alpha$, IL- $1 \beta$, and IL-6 expression, as well as elevated microglial activation (Cadet et al., 1994; Lai et al., 2009; Gonçalves et al., 2010). Cocaine has similarly been associated with increases in TNF- $\alpha$, IL-6, IL-8, activator protein 1 (AP-1), and nuclear factor kappa B (NFкB) (Zhang et al., 1998; Gan et al., 1999; Lee et al., 2001; Dhillon et al., 2008). Nicotine is marked by increases in TNF$\alpha$, IL-18, IL-1 $\beta$, and chemokines, including CCL2, CCL8, and CXC3CL1 (Bradford et al., 2011). Pro-inflammatory agents, such as stimulants, have also been associated with deterioration of the natural obstacle that protects the brain; the blood brain barrier, further magnifying their neurotoxic effects (Czub et al., 2001; Nath et al., 2002). MOD has been shown to counteract the toxic and neuroinflammatory effects of METH in mice (Raineri et al., 2012), but effects against other drugs of abuse have yet to be reported.

Modafinil administration has also been shown to exert effects on histamine, a common marker of inflammation and neurotransmitter involved in sleep/wakefulness (Haas et al., 2008). Using in vivo microdialysis, an increased histamine release in the anterior hypothalamus was observed following MOD administration (Ishizuka et al., 2003).

\section{PRECLINICAL STUDIES ON MOD AS A PHARMACOTHERAPEUTIC TREATMENT FOR PSUD}

\section{Neurochemical Studies}

In this section, we will review the neurochemistry of MOD as it relates to PSUD. The main pharmacologic activity of MOD is due to its affinity and inhibitory actions at DAT, which result in stimulation of brain extracellular DA levels. DAT and the DA system also play a major role in the abuse liability of psychostimulants. Thus, we will start this section with a brief background about DAT and DA roles in PSUD. 
DA and DAT, Their Role in Drug Abuse, Dependence, and as Potential Targets for Pharmacotherapy of PSUD

Dopamine's role in the brain's reward circuit has been extensively studied (Wise and Rompre, 1989; Di Chiara et al., 1993a, 1998; Wise, 2008; Arias-Carrión et al., 2010; Taber et al., 2012), however its role in drug abuse and dependence is still not fully clarified (Volkow et al., 2011; Wise and Robble, 2020). Following acute administration of drugs of abuse, including central stimulants and depressants, opiates, cannabinoids, and cholinergic agonists, increased levels of extracellular DA have been reported in the brain regions that are the projection fields of dopaminergic neurons, specifically the NAcc and caudate (Di Chiara and Imperato, 1988; Koob, 1992; Pontieri et al., 1995, 1996; Tanda et al., 1997a; Di Chiara et al., 1999). Acute administration of psychostimulants, in particular, has been shown to increase DA levels in a dose dependent manner in the NAcc shell and core, and in the striatum (Di Chiara et al., 1993b; Pontieri et al., 1995; Tanda et al., 1997b). These effects are likely related to the initial positive experience of drug use that could also lead to acquisition of drug-seeking behaviors and to the desire to repeat behaviors that lead to a pleasurable experience (Pettit and Justice, 1989; Woolverton and Johnson, 1992; Koob et al., 1998), but do not account for all neurological aspects of substance use disorder (Salamone et al., 2003; Robinson and Kolb, 2004; Russo et al., 2009; Golden and Russo, 2012). Repeated drug use has been shown to cause synaptic changes, allowing for the development of a different regulation of neurotransmission and other neuronal activities, which is believed to be the driving force behind drug addiction (Thomas et al., 2008; Luscher and Malenka, 2011). Indeed, addictive drugs consistently elicit neurological changes that are indicative of potential targets for better understanding and treating the development of specific patterns of drug use and dependence.

Regulation of expression and trafficking of presynaptic DATs by synaptic DA levels has been proposed as a pharmacological target involved in the development of PSUD (Zahniser and Sorkin, 2004). Indeed, both acute and chronic cocaine exposure increases DAT density in the NAcc and DS (Zahniser and Sorkin, 2004), while other psychostimulants such as amphetamine and METH decrease DAT expression in the same regions (Saunders et al., 2000; Sandoval et al., 2001; Barr et al., 2006; Kahlig et al., 2006). Despite varying levels of transporter presence, a primary result of psychostimulant use is an increase in synaptic DA levels by inhibiting its presynaptic neuronal reuptake or by interacting with the VMAT2, releasing DA into the cytoplasm and then releasing DA into the synapse by reversing its transport direction through DAT (Sulzer et al., 2005; Xie and Miller, 2009; Calipari et al., 2013). The regulation of DAT expression allows the formation of a feedback loop between DAT abundance and psychostimulant presence in the brain (Verma, 2015). The resulting changes in DAT density after drug use perpetuates a need for consistent amounts of the drug to avoid withdrawal and to maintain significant levels of DA and DAT expression.

TABLE 2 | Neurochemical actions of MOD.

\begin{tabular}{|c|c|c|c|}
\hline Agent(s) & Dose(s), species & Effect of MOD & References \\
\hline \multirow[t]{6}{*}{ MOD } & $\begin{array}{l}\text { 3-300 mg/kg, s.c. } \\
\text { RAT }\end{array}$ & - $\uparrow$ Extracellular NAcc DA levels & Ferraro et al. (1996c) \\
\hline & $\begin{array}{l}20-60 \mathrm{mg} / \mathrm{kg} \text {, i.v. } \\
\text { RAT }\end{array}$ & $\begin{array}{l}-\uparrow \text { Extracellular DA in the NAcc } \\
-\downarrow \text { METH-induced stimulation of NAcc DA levels }\end{array}$ & Zolkowska et al. (2009) \\
\hline & $\begin{array}{l}10-56 \mathrm{mg} / \mathrm{kg} \text {, i.v. } \\
\text { RAT }\end{array}$ & $\begin{array}{l}\text { - } \uparrow \text { Extracellular NAS DA levels } \\
\text { - NSE on cocaine-induced stimulation of NAS DA levels }\end{array}$ & Mereu et al. (2020) \\
\hline & $\begin{array}{l}10 \mu \mathrm{g} / 5 \mu \mathrm{L}, \text { i.c.v. } \\
\text { RAT }\end{array}$ & $-\uparrow$ Extracellular NAcc DA levels & $\begin{array}{l}\text { Murillo-Rodríguez et al. } \\
\text { (2007) }\end{array}$ \\
\hline & $\begin{array}{l}\text { 100-600 mg/kg, p.o. } \\
\text { RAT }\end{array}$ & - $\uparrow$ Extracellular DA in the striatum and PFC & Rowley et al. (2014) \\
\hline & $\begin{array}{l}\text { 30-300 mg/kg, i.p. } \\
\text { RAT }\end{array}$ & - $\uparrow$ Electrically evoked DA in the ventral and dorsal striatum & Bobak et al. (2016) \\
\hline \multirow[t]{4}{*}{ R-MOD } & $\begin{array}{l}\text { 30-100 mg/kg, i.p. } \\
\text { RAT }\end{array}$ & $\begin{array}{l}-\uparrow \text { Extracellular DA in the NAcc } \\
-\downarrow \text { Nicotine-induced stimulation of NAcc DA levels }\end{array}$ & Wang et al. (2015) \\
\hline & $\begin{array}{l}\text { 30-300 mg/kg, i.p. } \\
\text { MOUSE }\end{array}$ & - $\uparrow$ Extracellular NAS DA levels & Loland et al. (2012) \\
\hline & $\begin{array}{l}\text { 10-32 mg/kg, i.v. } \\
\text { MOUSE }\end{array}$ & $\begin{array}{l}-\uparrow \text { Extracellular NAS DA levels } \\
-\uparrow \text { Electrically evoked NAS DA } \\
-\downarrow \text { DA clearance rate }\end{array}$ & Keighron et al. (2019a) \\
\hline & $\begin{array}{l}5-100 \mathrm{mg} / \mathrm{kg}, \text { i.p. } \\
\text { MOUSE }\end{array}$ & $\begin{array}{l}-\uparrow \text { Electrically evoked DA in the NAS } \\
-\downarrow \text { DA clearance rate }\end{array}$ & Keighron et al. (2019b) \\
\hline S-MOD & $\begin{array}{l}\text { 30-300 mg/kg, i.p. } \\
\text { MOUSE }\end{array}$ & - $\uparrow$ Extracellular NAS DA levels & $\begin{array}{l}\text { Loland et al. (2012); Mereu } \\
\text { et al. (2020) }\end{array}$ \\
\hline
\end{tabular}

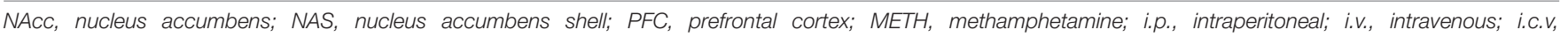
intracerebroventricular injection; s.c., subcutaneous; p.o., oral administration; NSE, not a significant effect; $\uparrow$, increase; $\downarrow$, decrease. 


\section{DA and DAT as Potential Pharmacologic Target for the Therapeutic Actions of MOD Against PSUD}

The use of MOD as a therapeutic agent for PSUD is largely based on its mechanistic actions that, in part, overlap with those of other abused psychostimulants. For instance, abused psychostimulants increase mesolimbic extracellular DA, often by interacting with DAT (Mortensen and Amara, 2003; Zhu and Reith, 2008), and MOD has been shown to stimulate DA levels in the same dopaminergic areas related to psychostimulant actions (Ferraro et al., 1996c; Zolkowska et al., 2009; Loland et al., 2012; Rowley et al., 2014; Bobak et al., 2016; Mereu et al., 2020). Even though the pharmacological actions of MOD have been mainly explained by its affinity for DAT, its unique psychostimulant profile has been shown to differ from that of typical DAT inhibitors, as shown in behavioral, neurochemical and molecular pharmacology studies (Schmitt and Reith, 2011; Loland et al., 2012; Mereu et al., 2013, 2017, 2020). For example, MOD binding to DAT differs from that of other typical, cocaine-like, DAT blockers (Schmitt and Reith, 2011). In contrast to cocaine, MOD prefers to bind to, or stabilize the DAT protein in a more inwardfacing occluded conformation (Schmitt and Reith, 2011; Loland et al., 2012) that still inhibits uptake and results in increases in extracellular DA in the NAcc (Ferraro et al., 1996c; Zolkowska et al., 2009), the NAcc shell (NAS) (Loland et al., 2012; Mereu et al., 2020), and the striatum (Rowley et al., 2014). MOD also increases electrically evoked DA in the DS and VS (Bobak et al., 2016) (summarized in Table 2) like abused psychostimulants (Nisell et al., 1994; Pontieri et al., 1996; Munzar et al., 2004; Kohut et al., 2014). However, while acute administration of MOD (Mereu et al., 2017, 2020) or its enantiomers (Loland et al., 2012; Keighron et al., 2019a,b) increases extracellular NAcc DA levels in rodents, these effects, even at very high doses, elicited a limited stimulation of DA in striatal areas compared to the stimulation elicited by abused psychostimulants (Loland et al., 2012; Mereu et al., 2017, 2020). This limited efficacy of MOD to increase DA levels, as compared to abused psychostimulants, also predicts a limited potential for abuse.

Cocaine psychostimulant actions and its abuse liability have been related to its ability to slow DA reuptake by inhibiting DAT and stimulating DA neurotransmission (Wise and Bozarth, 1987; Kuhar et al., 1991). It is interesting to note that administration of MOD (10-32 mg/kg, i.p.) prior to cocaine produced no further increase in extracellular NAS DA levels beyond that produced by cocaine alone (Mereu et al., 2020). This effect varied with the additive effects on DA levels obtained with combinations of cocaine and typical DAT blockers like methylphenidate or WIN 35,428 (Tanda et al., 2009; Mereu et al., 2020), but similar to the effects shown by combinations of cocaine and an atypical DAT blocker like JHW007 (Tanda et al., 2009), suggesting a potential atypical DAT inhibitor effect for MOD in these tests.

Another abused psychostimulant, METH, is transported into DA neurons and its nerve terminals as a DAT substrate, like DA, where it has also been shown to affect the VMAT2 function. As a consequence, decreased vesicular DA concentrations and increased cytoplasmic DA levels result, via reverse transport of DA through DAT (Kahlig and Galli, 2003; Sulzer et al., 2005; Howell and Kimmel, 2008), resulting in dramatic increases in extracellular DA levels and robust stimulation of behavioral activities (Munzar et al., 2004). When administered prior to METH, MOD significantly attenuated the stimulatory effects of METH on extracellular NAcc DA levels (see Table 2) (Zolkowska et al., 2009). This effect suggests the possibility that blockade of DAT by MOD pretreatment could affect the ability of METH to be transported by DAT as its substrate into the DA nerve terminal, thus reducing its ability to enhance extracellular DA levels. Reducing the dopaminergic effects of METH could play a role in the therapeutic effects shown by MOD in some preclinical behavioral reports and in clinical studies on METH dependent subjects.

Nicotine, the key addictive component in tobacco, exerts indirect actions on DAT. Voltammetry studies revealed that nicotine slows DA clearance (Hart and Ksir, 1996), in addition to nicotine's actions in modulating dopaminergic transmission via activation of nicotinic acetylcholine receptors on DA neurons (Clarke and Pert, 1985; Picciotto et al., 1998; Laviolette and Van Der Kooy, 2004). When administered prior to nicotine, MOD produced a reduction in nicotine-induced stimulation of extracellular NAcc DA levels (see Table 2) (Wang et al., 2015).

These preclinical actions of MOD as an atypical DAT inhibitor suggest a strong potential for its therapeutic use in PSUDs (see Table 2).

\section{Modulation of Brain Glutamate Levels by MOD Plays a Role in Its Therapeutic Actions on PSUD}

The excitatory neurotransmitter, glutamate, has long been associated with many brain physiological functions and brain diseases including addiction (Meldrum, 2000; Kalivas, 2009). Interestingly, the effects of MOD administration on glutamate levels varies by brain region (reviewed in Gerrard and Malcolm, 2007; Mereu et al., 2013). It is predicted that this could be due, in part, to corresponding activation/inactivation of the inhibitory neurotransmitter, GABA. MOD produced increases in glutamate in the medial preoptic areas (Ferraro et al., 1996b), posterior hypothalamus (Ferraro et al., 1996b), thalamus (Ferraro et al., 1997a), hippocampus (Ferraro et al., 1997a), and striatum (Ferraro et al., 1996a, 1998). It was only at high does $(300 \mathrm{mg} / \mathrm{kg}$ MOD) that increases in glutamate were observed in the substantia nigra or the pallidum (Ferraro et al., 1998). MOD also shows agonist activity at some glutamate receptors (group II metabotropic; mGlu2/3) (TahsiliFahadan et al., 2010), although this is likely not due to direct receptor activation. Behaviorally, the impaired reinstatement of extinguished CPP for opiates following MOD administration was blunted with an mGlu2/3 antagonist pretreatment (TahsiliFahadan et al., 2010). Neurochemically, cystine-glutamate exchange or voltage dependent calcium channel antagonist administration blocked increases in glutamate in the NAcc following $\mathrm{MOD}$, in rats chronically trained to self-administer cocaine (Mahler et al., 2014).

The effects of MOD on glutamate can be directly linked to many of the agent's biological effects. For example, MOD-produced increases in synaptic plasticity and long-term potentiation of glutamatergic connections to orexin neurons in the lateral hypothalamus is linked to improved wakefulness and 
cognition (Rao et al., 2007), but it is also linked to drug reinforced behaviors (Boutrel et al., 2013).

\section{Effects of MOD on Behavioral Models of PSUD}

Herein, we will review animal preclinical data on behavioral tests, mainly in rodents, used to model specific aspects of human substance use disorders, especially PSUD. Importantly, we will compare results from reports analyzing the effects of psychostimulants alone, MOD alone, and MOD in combination with psychostimulants, as summarized in Table 3.

\section{Locomotion, Stereotypy, and Behavioral Sensitization}

Acute administration of psychostimulant drugs of abuse generally produces a dose-dependent stimulation of exploratory behaviors, including locomotion and stereotyped movements in rodents (Sahakian et al., 1975). Repeated administration of psychostimulants might result in behavioral sensitization (Kalivas and Duffy, 1993; Mereu et al., 2015), a phenomena related to neurobiological adaptations (Ghasemzadeh et al., 2009; Bowers et al., 2010), which lead to a heightened behavioral response to a psychostimulant. The potential of novel drugs to cause sensitization can be indicative of their potential neurological long-term effects that could be related to the development of drug dependence (Kauer and Malenka, 2007).

Modafinil administered alone induced dose-dependent changes in locomotion and stereotyped movements in rats (Zolkowska et al., 2009; Chang et al., 2010; Alam and Choudhary, 2018) and mice (Paterson et al., 2010; Wuo-Silva et al., 2011, 2016; Young et al., 2011), with similar results found in response to R-MOD (Zhang et al., 2017). However, a report by Shuman et al. (2012) found no significant change in locomotion in mice treated with both low and high doses of MOD (Shuman et al., 2012). In rhesus monkeys, nighttime locomotion increased, but daytime locomotion had no significant effect (Andersen et al., 2010), calling into question whether the behaviors measured in these assays are due to the same mechanisms as psychostimulant drugs, or if it is a by-product of the primary wake inducing effects of MOD (Chang et al., 2010). In another report, when locomotion was tested relative to time spent awake in rats, the time awake increased, but locomotor activity only increased for the lowest dose administered (30 mg/kg) (Wisor et al., 2006).

The locomotor activating effects of MOD have also been tested in combination with several psychiatric medications and abused psychostimulants that affect brain neurotransmission at different levels. Haloperidol, a DA D2 receptor antagonist and a commonly prescribed antipsychotic medication, decreased MOD induced locomotion in rats (Alam and Choudhary, 2018), indicating a potential interaction between MOD-induced stimulation of DA levels by blockade of DAT, and inhibition of DA transmission due to blockade of DA D2 receptors by haloperidol. Further, these effects suggest the potential interactions of medications for mental disorders and addiction, which are often found comorbidly. A pretreatment with MOD did not produce significant alteration in cocaine-induced locomotion in mice (Shuman et al., 2012), but MOD significantly decreased METH induced locomotion in rats (Zolkowska et al., 2009), indicating a lack of compounding effects on locomotor activities of MOD in the latter report, which could be dependent on differences in the specific mechanisms of action between different stimulants: cocaine is a DAT blocker, while METH is a DAT substrate and a blocker of the vesicular VMAT2 transporter.

It has been reported that repeated MOD exposure in rats (Chang et al., 2010) and mice (Paterson et al., 2010; Wuo-Silva et al., 2011) would induce behavioral sensitization of locomotion and stereotyped movements, which is further enhanced by exposure to stress (Alam and Chaudhary, 2020). Also, clear individual differences in responses of mice to MODinduced sensitization have been found (da Costa Soeiro et al., 2012), indicating the importance of better understanding how these differences may lead to individualized treatment. Rapidonset sensitization was decreased by DA antagonists $\mathrm{SCH} 23390$ and sulpiride (Wuo-Silva et al., 2019), and behavioral crosssensitization was induced between MOD and apomorphine, a direct DA agonist (Chang et al., 2010). MOD administered with cocaine (Wuo-Silva et al., 2011, 2016; Shuman et al., 2012) or METH (da Costa Soeiro et al., 2012) also caused bidirectional sensitization in mice, indicating similar neurological effects of these drugs. While these results require further validation, they may indicate possible neuronal plasticity, which for some drugs has been suggested to have a role in their dependence producing actions (Kauer and Malenka, 2007).

\section{Conditioned Place Preference}

Drug CPP paradigms consist of classically conditioning an animal to associate a contextually unique location (chamber) with administration of a drug reinforcer, while a different chamber is associated with administration of the reinforcer's vehicle. After training, animals are given the opportunity to freely explore the distinct locations previously associated with administration of the reinforcer or its vehicle. Assessing the difference in time spent by animals in the two chambers would provide an index of their preference (potentially drug-seeking behavior), indifference, or even aversion toward the chamber associated with the reinforcer (Tzschentke, 2007). Induction of CPP can be obtained by administration of specific doses of drugs of abuse, for example psychostimulants, such as cocaine (Mueller and Stewart, 2000; Itzhak and Martin, 2002) and METH (Itzhak and Martin, 2002), but can also be obtained through illicit drugs (Liu et al., 2008) and other natural reinforcers such as palatable foods (VelázquezSánchez et al., 2015). Therefore, CPP is a common preclinical assay that could be used to assess the potential pleasurable effects of a novel medication and to evaluate its potential for abuse.

Modafinil administered alone was unable to induce CPP in rats when administered orally (Deroche-Gamonet et al., 2002), or by intraperitoneal injection (Tahsili-Fahadan et al., 2010; Quisenberry et al., 2013), in contrast with results found in mice (Nguyen et al., 2011; Wuo-Silva et al., 2011; Shuman et al., 2012). These results indicate a minimal, if any, pleasurable effect of MOD, resulting in a low abuse liability for naïve subjects. However, the results in mice indicate a potential species difference, thus further investigation into various model species is required to thoroughly understand the effects of MOD. 
TABLE 3 | MOD effects on preclinical behavioral animal models related to PSUD.

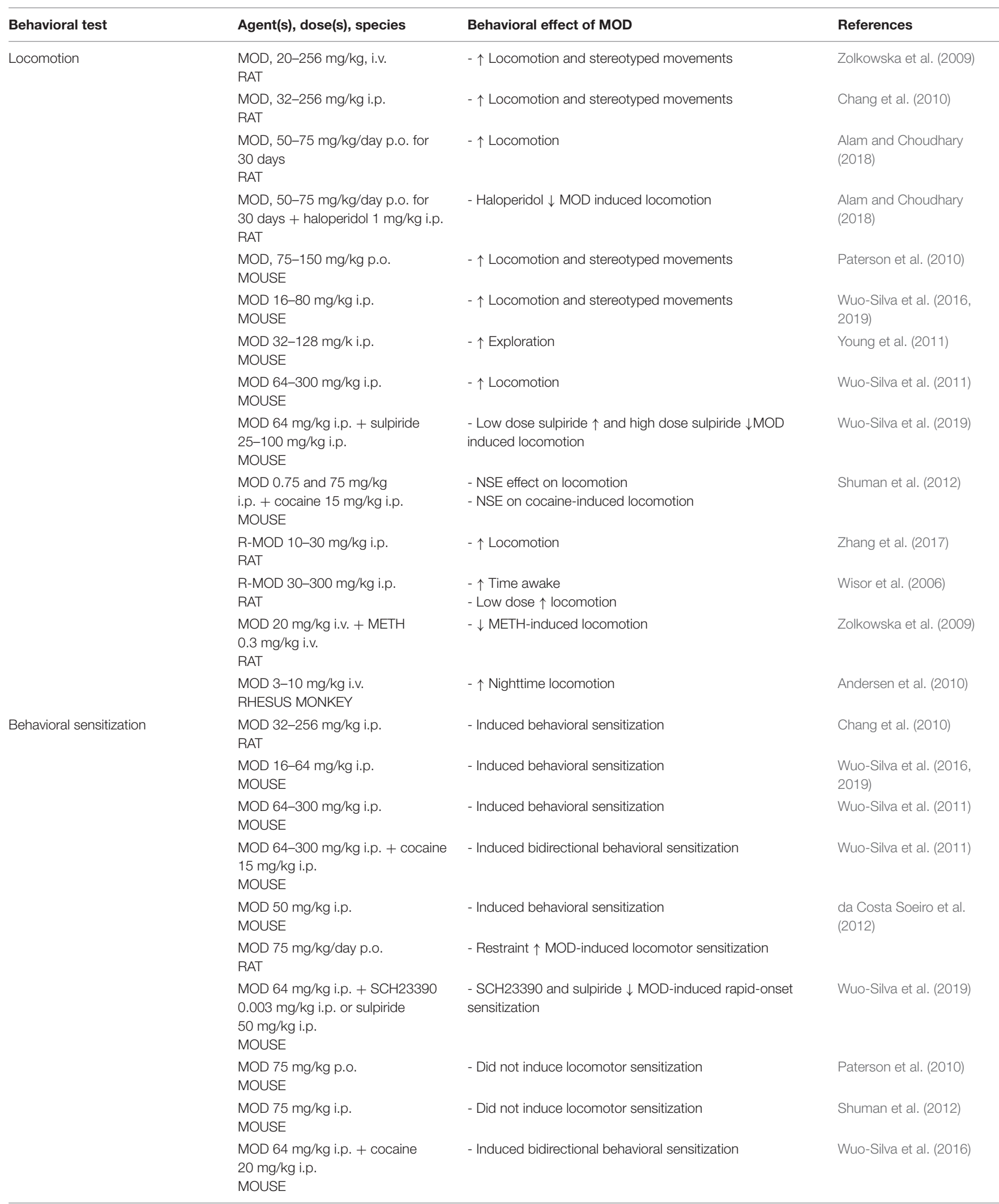


TABLE 3 | Continued

\begin{tabular}{|c|c|c|c|}
\hline Behavioral test & Agent(s), dose(s), species & Behavioral effect of MOD & References \\
\hline & $\begin{array}{l}\text { MOD } 50 \mathrm{mg} / \mathrm{kg} \text { i.p. }+ \text { METH } \\
1 \mathrm{mg} / \mathrm{kg} \text { i.p. } \\
\text { MOUSE }\end{array}$ & - Induced cross-sensitization & $\begin{array}{l}\text { da Costa Soeiro et al. } \\
(2012)\end{array}$ \\
\hline & $\begin{array}{l}\text { MOD } 75 \text { mg/kg i.p. + cocaine } \\
15 \text { mg/kg i.p. } \\
\text { MOUSE }\end{array}$ & - Induced cross-sensitization & Shuman et al. (2012) \\
\hline \multirow[t]{7}{*}{ Conditioned place preference } & $\begin{array}{l}\text { MOD } 32-300 \mathrm{mg} / \mathrm{kg} \text { i.p. } \\
\text { RAT }\end{array}$ & - Did not induce CPP & $\begin{array}{l}\text { Deroche-Gamonet et al. } \\
\text { (2002); Tahsili-Fahadan } \\
\text { et al. (2010) }\end{array}$ \\
\hline & $\begin{array}{l}\text { MOD } 64 \text { mg/kg p.o. } \\
\text { RAT }\end{array}$ & - Did not induce CPP & Quisenberry et al. (2013) \\
\hline & $\begin{array}{l}\text { MOD } 125 \text { mg/kg i.p. } \\
\text { MOUSE }\end{array}$ & - Induced CPP & Nguyen et al. (2011) \\
\hline & $\begin{array}{l}\text { MOD 64-300 mg/kg i.p. } \\
\text { MOUSE }\end{array}$ & - Induced CPP & Wuo-Silva et al. (2011) \\
\hline & $\begin{array}{l}\text { MOD } 0.75 \text { and } 75 \text { mg/kg } \\
\text { i.p. + cocaine } 15 \mathrm{mg} / \mathrm{kg} \text { i.p. } \\
\text { MOUSE }\end{array}$ & $\begin{array}{l}\text { - Induced CPP } \\
\text { - NSE on cocaine induced CPP }\end{array}$ & Shuman et al. (2012) \\
\hline & $\begin{array}{l}\text { MOD } 128 \mathrm{mg} / \mathrm{kg} \text { i.p. + cocaine } \\
20 \mathrm{mg} / \mathrm{kg} \text { i.p. } \\
\text { RAT }\end{array}$ & - Reinstated cocaine CPP & Bernardi et al. (2009) \\
\hline & $\begin{array}{l}\text { MOD } 300 \text { mg/kg i.p. + morphine } \\
\text { 8-16 mg/kg i.p. } \\
\text { RAT }\end{array}$ & - Did not reinstate morphine CPP & Tahsili-Fahadan et al. (2010) \\
\hline \multirow[t]{11}{*}{ Self-administration } & $\begin{array}{l}\text { MOD 0.28-1.7 mg/kg/inj i.v. } \\
\text { RAT }\end{array}$ & - Did not induce self-administration & $\begin{array}{l}\text { Deroche-Gamonet et al. } \\
\text { (2002) }\end{array}$ \\
\hline & $\begin{array}{l}\text { MOD 0.1-10 mg/kg/inj i.v. } \\
\text { RAT }\end{array}$ & - Did not maintain self-administration in cocaine-trained rats & Mereu et al. (2020). \\
\hline & $\begin{array}{l}\text { MOD } 10-32 \mathrm{mg} / \mathrm{kg} \text { i.p. + cocaine } \\
0.03-1 \mathrm{mg} / \mathrm{kg} / \mathrm{inj} \\
\text { RAT }\end{array}$ & - $\uparrow$ Cocaine self-administration for lowest dose of cocaine & Mereu et al. (2020). \\
\hline & $\begin{array}{l}\text { MOD } 32-128 \mathrm{mg} / \mathrm{kg} \text { i.p. + cocaine } \\
0.25-1 \mathrm{mg} / \mathrm{kg} / \mathrm{inj} \\
\text { RAT }\end{array}$ & - NSE on cocaine self-administration & $\begin{array}{l}\text { Deroche-Gamonet et al. } \\
\text { (2002) }\end{array}$ \\
\hline & $\begin{array}{l}\text { R-MOD } 10-100 \mathrm{mg} / \mathrm{kg} \\
\text { i.p. + cocaine } 0.5-1 \mathrm{mg} / \mathrm{kg} / \mathrm{inj} \text { i.v. } \\
\text { RAT }\end{array}$ & $\begin{array}{l}\text { - NSE on cocaine self-administration } \\
\text { - } \downarrow \text { Reinstatement of cocaine seeking at high doses }\end{array}$ & Zhang et al. (2017) \\
\hline & $\begin{array}{l}\text { R-MOD/S-MOD 30-100 mg/kg } \\
\text { i.p. + nicotine } 7.5-60 \mu \mathrm{g} / \mathrm{kg} / \mathrm{inj} \text { i.v. } \\
\text { RAT }\end{array}$ & $\begin{array}{l}\text { - R-MOD } \downarrow \text { nicotine self-administration } \\
\text { - S-MOD } \downarrow \text { nicotine self-administration at high doses }\end{array}$ & Wang et al. (2015) \\
\hline & $\begin{array}{l}\text { MOD } 30-300 \mathrm{mg} / \mathrm{kg} \text { i.p. + METH } \\
20 \mu \mathrm{g} / 50 \mu \mathrm{L} \text { bolus i.v. inj } \\
\text { RAT }\end{array}$ & - $\downarrow$ METH self-administration & Reichel and See (2012) \\
\hline & $\begin{array}{l}\text { R-MOD } 100 \mathrm{mg} / \mathrm{kg} \text { i.p. + METH } \\
0.05 \mathrm{mg} / \mathrm{kg} / \mathrm{inj} \text { i.v. } \\
\text { RAT }\end{array}$ & $\begin{array}{l}\text { - } \downarrow \text { METH self-administration when given } 1-h \text { access to } \\
\text { METH } \\
\text { - NSE on self-administration when given } 6 \text {-h access to } \\
\text { METH }\end{array}$ & Tunstall et al. (2018) \\
\hline & $\begin{array}{l}\text { MOD 30-300 mg/kg i.p. + METH } \\
20 \mu \mathrm{g} / 50 \mu \mathrm{L} \text { bolus inj or } \\
\text { O.05 mg/kg/inj i.v. } \\
\text { RAT }\end{array}$ & $\begin{array}{l}\text { - Did not reinstate METH self-administration } \\
\text { - } \downarrow \text { Cue, drug, and context induced reinstatement }\end{array}$ & $\begin{array}{l}\text { Reichel and See (2010, } \\
\text { 2012); Holtz et al. (2012) }\end{array}$ \\
\hline & $\begin{array}{l}\text { MOD } 32 \mathrm{mg} / \mathrm{kg} / \text { day i.v. for } \\
7-10 \text { days }+ \text { cocaine } \\
0.001-0.1 \mathrm{mg} / \mathrm{kg} / \text { inj i.v. } \\
\text { RHESUS MONKEY }\end{array}$ & - MOD $\downarrow$ cocaine self-administration & Newman et al. (2010) \\
\hline & $\begin{array}{l}\text { MOD 32-56 mg/kg/day i.v. after } \\
\text { extinction of cocaine-maintained } \\
\text { self-administration } \\
\text { RHESUS MONKEY }\end{array}$ & - MOD reinstated cocaine self-administration behavior & Newman et al. (2010) \\
\hline
\end{tabular}


TABLE 3 | Continued

\begin{tabular}{|c|c|c|c|}
\hline Behavioral test & Agent(s), dose(s), species & Behavioral effect of MOD & References \\
\hline & $\begin{array}{l}\text { MOD } 0.03-0.3 \mathrm{mg} / \mathrm{kg} \text { i.v., cocaine } \\
0.02-0.05 \mathrm{mg} / \mathrm{kg} / \mathrm{inj} \text { i.v. } \\
\text { RHESUS MONKEY }\end{array}$ & $\begin{array}{l}-\uparrow \text { Responding in cocaine-trained subjects switched to } \\
\text { MOD }\end{array}$ & Gold and Balster (1996) \\
\hline & $\begin{array}{l}\text { MOD 3-10 mg/kg i.v. + cocaine } \\
0.1 \mathrm{mg} / \mathrm{kg} / \mathrm{inj} \text { i.v. } \\
\text { RHESUS MONKEY }\end{array}$ & - High dose induced reinstatement & Andersen et al. (2010) \\
\hline \multirow[t]{3}{*}{ Intracranial self-stimulation } & $\begin{array}{l}\text { MOD } 20-600 \mathrm{mg} / \mathrm{kg} \text { p.o. } \\
\text { RAT }\end{array}$ & - Facilitate ICSS & Lazenka and Negus (2017) \\
\hline & $\begin{array}{l}\text { MOD } 50-150 \mathrm{mg} / \mathrm{kg} \text { i.p. } \\
\text { RAT }\end{array}$ & - Facilitate ICSS & Burrows et al. (2015) \\
\hline & $\begin{array}{l}\text { R-MOD } 50-150 \mathrm{mg} / \mathrm{kg} \text { i.p. } \\
\text { RAT }\end{array}$ & - Trend toward facilitating ICSS & Burrows et al. (2015) \\
\hline \multirow[t]{5}{*}{ Drug discrimination } & $\begin{array}{l}\text { MOD 3-300 mg/kg i.p., } \\
\text { cocaine-trained } 10 \mathrm{mg} / \mathrm{kg} \text { i.p. } \\
\text { RATS }\end{array}$ & - $67 \%$ cocaine substitution at doses above 100 mg/kg & $\begin{array}{l}\text { Gold and Balster (1996); } \\
\text { Paterson et al. (2010) }\end{array}$ \\
\hline & $\begin{array}{l}\text { MOD } 30-300 \text { mg/kg i.p., } \\
\text { cocaine-trained } 10 \mathrm{mg} / \mathrm{kg} \text { i.p. } \\
\text { RAT }\end{array}$ & - Full substitution at $300 \mathrm{mg} / \mathrm{kg}$ & Paterson et al. (2010) \\
\hline & $\begin{array}{l}\text { MOD } 10-100 \mathrm{mg} / \mathrm{kg} \text { i.p., } \\
\text { cocaine-trained } 10 \mathrm{mg} / \mathrm{kg} \text { i.p. } \\
\text { MICE }\end{array}$ & $\begin{array}{l}\text { - Substitution approached } 80 \% \text { at MOD } 56-100 \text { mg/kg } \\
\text { administered } 5 \text { min before testing, and 100\% when } \\
\text { administered } 60 \text { min before testing }\end{array}$ & Mereu et al. (2017) \\
\hline & $\begin{array}{l}\text { R-MOD/S-MOD } 10-100 \mathrm{mg} / \mathrm{kg} \text { i.p., } \\
\text { cocaine-trained } 10 \mathrm{mg} / \mathrm{kg} \text { i.p. } \\
\text { MICE }\end{array}$ & - Full substitution & Loland et al. (2012) \\
\hline & $\begin{array}{l}\text { MOD } 3.2-32 \mathrm{mg} / \mathrm{kg} \text { i.m., } \\
\text { cocaine-trained } 0.18-0.4 \mathrm{mg} / \mathrm{kg} \\
\text { i.m. } \\
\text { RHESUS MONKEYS }\end{array}$ & - $75 \%$ substitution & Newman et al. (2010) \\
\hline \multirow[t]{10}{*}{ Cognitive function } & $\begin{array}{l}\text { MOD } 64 \mathrm{mg} / \mathrm{kg} \\
\text { p.o. + phencyclidine } 5 \mathrm{mg} / \mathrm{kg} \text { i.p. } \\
\text { twice daily for } 7 \text { days } \\
\text { RAT }\end{array}$ & $\begin{array}{l}\text { - Improved phencyclidine-induced object recognition } \\
\text { memory deficits }\end{array}$ & Redrobe et al. (2010) \\
\hline & $\begin{array}{l}\text { MOD } 30-90 \text { mg/kg i.p. + METH } \\
1 \mathrm{mg} / \mathrm{kg} \text { s.c. for } 7 \text { days } \\
\text { MOUSE }\end{array}$ & $\begin{array}{l}\text { - Improved METH induced object recognition memory } \\
\text { deficits }\end{array}$ & González et al. (2014) \\
\hline & $\begin{array}{l}\text { MOD } 100 \text { mg/kg i.p. + METH } \\
4 \text { mg/kg i.p. } 2 \text { h apart, } 4 \text { times/day } \\
\text { MOUSE }\end{array}$ & $\begin{array}{l}\text { - Improved METH induced object recognition memory } \\
\text { deficits }\end{array}$ & Reichel et al. (2014) \\
\hline & $\begin{array}{l}\text { MOD } 50-75 \mathrm{mg} / \mathrm{kg} / \text { day p.o. for } \\
30 \text { days } \\
\text { RAT }\end{array}$ & $\begin{array}{l}\text { - } \uparrow \text { Memory on Morris Water Maze and passive avoidance } \\
\text { task }\end{array}$ & $\begin{array}{l}\text { Alam and Choudhary } \\
\text { (2018) }\end{array}$ \\
\hline & $\begin{array}{l}\text { MOD } 50-75 \text { mg/kg/day p.o. for } \\
30 \text { days }+ \text { haloperidol } 1 \mathrm{mg} / \mathrm{kg} \text { i.p. } \\
\text { RAT }\end{array}$ & $\begin{array}{l}\text { - Haloperidol } \downarrow \text { MOD effects on Morris Water Maze and on } \\
\text { passive avoidance task }\end{array}$ & $\begin{array}{l}\text { Alam and Choudhary } \\
\text { (2018) }\end{array}$ \\
\hline & $\begin{array}{l}\text { MOD } 0.75-75 \mathrm{mg} / \mathrm{kg} \text { i.p. } \\
\text { MOUSE }\end{array}$ & $\begin{array}{l}\text { - } \uparrow \text { Learning rate and memory on Morris Water Maze } \\
\text { - Low dose } \uparrow \text { memory of contextual fear conditioning } \\
\text { - High dose } \downarrow \text { memory of contextual fear conditioning } \\
\text { - NSE on cued conditioning }\end{array}$ & Shuman et al. (2009) \\
\hline & $\begin{array}{l}\text { MOD } 0.75-75 \mathrm{mg} / \mathrm{kg} \text { i.p. } \\
\text { RAT }\end{array}$ & - NSE on memory of naïve subjects & Garcia et al. (2013) \\
\hline & $\begin{array}{l}\text { MOD } 64 \mathrm{mg} / \mathrm{kg} \text { p.o. }+20 \% \text { alcohol } \\
\text { (4-6 g/kg/day prenatally, } 21 \text { days) } \\
\text { RAT }\end{array}$ & $\begin{array}{l}-\downarrow \text { Impulsive responses in healthy and prenatal } \\
\text { alcohol-treated rats } \\
\text { - } \downarrow \text { Response latency in prenatal alcohol-treated rats only. }\end{array}$ & $\begin{array}{l}\text { Heyer-Osorno and Juárez } \\
\text { (2020) }\end{array}$ \\
\hline & $\begin{array}{l}\text { MOD 32-64 mg/kg i.p. for } 5 \text { days } \\
\text { MOUSE }\end{array}$ & $\begin{array}{l}\text { - } \uparrow \text { Speed of learning } \\
\text { - NSE on spatial working memory }\end{array}$ & Béracochéa et al. (2002) \\
\hline & $\begin{array}{l}\text { MOD } 64 \mathrm{mg} / \mathrm{kg} \text { i.p. for } 15 \text { days } \\
\text { RAT }\end{array}$ & $\begin{array}{l}\text { - NSE on working memory errors } \\
-\downarrow \text { Long-term memory errors } \\
-\downarrow \text { Successes in learning task }\end{array}$ & Burgos et al. (2010) \\
\hline
\end{tabular}

(Continued) 
TABLE 3 | Continued

\begin{tabular}{|c|c|c|c|}
\hline Behavioral test & Agent(s), dose(s), species & Behavioral effect of MOD & References \\
\hline & $\begin{array}{l}\text { R-MOD } 1-10 \mathrm{mg} / \mathrm{kg} \text { i.p. + DMSO } \\
1 \mathrm{~mL} / \mathrm{kg} \text { i.p. } \\
\text { RAT }\end{array}$ & $\begin{array}{l}\text { - Rescued DMSO-induced deficits in hole-board task } \\
\text { - NSE on normal functioning subjects }\end{array}$ & $\begin{array}{l}\text { Shanmugasundaram et al. } \\
\text { (2017) }\end{array}$ \\
\hline & $\begin{array}{l}\text { MOD 32-64 mg/kg i.p. } \\
\text { MOUSE }\end{array}$ & - $\uparrow$ Speed of learning & Béracochéa et al. (2003) \\
\hline & $\begin{array}{l}\text { MOD 30-100 mg/kg i.p. } \\
\text { RAT }\end{array}$ & - $\uparrow$ Speed of learning at high doses & Ward et al. (2004) \\
\hline & $\begin{array}{l}\text { MOD 8-64 mg/kg p.o. } \\
\text { RAT }\end{array}$ & $\begin{array}{l}-\uparrow \text { Visual attention } \\
-\uparrow \text { Impulse control } \\
-\downarrow \text { Premature responses } \\
\text { - NSE on visual discrimination performance }\end{array}$ & Morgan et al. (2007) \\
\hline & $\begin{array}{l}\text { MOD } 32-128 \text { mg/kg p.o. } \\
\text { RAT }\end{array}$ & $\begin{array}{l}\text { - NSE on attentional performance } \\
\text { - NSE on deficits induced by parametric or pharmacological } \\
\text { manipulations } \\
\text { - } \uparrow \text { Premature responding at high doses }\end{array}$ & Waters et al. (2005) \\
\hline & $\begin{array}{l}\text { MOD 3-100 mg/kg i.p. } \\
\text { RAT }\end{array}$ & $\begin{array}{l}\text { - } \downarrow \text { Reaction time in subjects with slow baseline reaction } \\
\text { times } \\
\text { - NSE on subjects with fast baseline reaction time }\end{array}$ & Eagle et al. (2007) \\
\hline
\end{tabular}

MOD, modafinil; METH, methamphetamine; CPP, conditioned place preference; ICSS, intracranial self-stimulation; i.p., intraperitoneal; i.v., intravenous; s.c., subcutaneous; p.o., oral administration; i.m., intramuscular; NSE, not a significant effect; $\uparrow$, increase; $\downarrow$, decrease.

The CPP assay can also be applied to understand whether MOD can reinstate seeking of the pleasurable effects of an extinguished behavioral response to a drug of abuse (Napier et al., 2013). Practically, the chamber previously associated with administration of the reinforcer is no longer associated to it, leading the subjects to forget the learned association and return to spending equal amounts of time in both chambers. Reinstatement of CPP occurs quickly following a single administration of the reinforcer. Administration of MOD alone has been shown to reinstate cocaine induced CPP in rats (Bernardi et al., 2009). In contrast to psychostimulants, opioid CPP is not reinstated by MOD treatment (Tahsili-Fahadan et al., 2010). These studies indicate a potential relapse inducing effect of MOD, which may be detrimental to PSUD target subjects. Further investigation is required to determine the varying effects of MOD on reinstatement of drug seeking for different drugs of abuse.

\section{Self-Administration}

The abuse potential of a substance can be assessed by using animal models of self-administration behavior, which could also model the transition from sampling or recreational use of a substance to its compulsive intake (Ator and Griffiths, 2003; Edwards and Koob, 2013). Compulsive selfadministration behavior in animals has been observed under specific experimental operational conditions when selected doses of psychostimulant drugs of abuse such as cocaine or amphetamines were made available (Deneau et al., 1969). In these models, the rate at which subjects would self-administer a substance could indicate the potential for abuse of a novel medication.

Modafinil (0.28-1.7 mg/kg/inj) (Deroche-Gamonet et al., 2002), alone did not promote intravenous self-administration behavior in naïve rats, indicating a lack of MOD reinforcing effects at the doses tested. Further, MOD alone (0.1$10 \mathrm{mg} / \mathrm{kg} / \mathrm{inj}$ ) did not maintain self-administration behavior in rats previously trained with intravenous doses of cocaine (Mereu et al., 2020), and similarly R-MOD was not self-administered in rats trained with nicotine (Wang et al., 2015). However, administration of MOD increased behavioral response rates for at least one dose for each subject in Rhesus monkeys previously trained to self-administer cocaine (Gold and Balster, 1996). This contradiction may be due to species differences or other procedural variables and requires further investigation.

Acute MOD treatment prior to psychostimulant selfadministration sessions might indicate whether MOD would affect the reinforcing effects of those drugs. MOD or R-MOD pretreatment did not affect intravenous cocaine selfadministration in rats (Deroche-Gamonet et al., 2002; Zhang et al., 2017). In contrast, a more recent study showed increased cocaine self-administration behavior at low cocaine doses (Mereu et al., 2020). Such effect was surprisingly not accompanied by enhancement of cocaine-induced stimulation of NAS DA levels, but it was reversed by pretreatments with carbenoxolone, an inhibitor of electrotonic coupling (Mereu et al., 2020). In rats, R-MOD has been shown to decrease METH (Tunstall et al., 2018) and nicotine self-administration behavior (Wang et al., 2015). Moreover, when MOD was administered chronically, it decreased cocaine self-administration responding in Rhesus monkeys (Newman et al., 2010).

After animals acquire and maintain self-administration behavior induced by abused psychostimulants, these behaviors can be extinguished by stopping drug-injections or eliminating conditioned stimuli associated with the availability or the injection of the drug. After extinction, it has been shown that non-contingent injections of the training drug or reintroduction of its associated cues can reinstate the operant behavior required to deliver the drug, suggesting a potential for relapse. These procedures could also assess the potential effects of test compounds, administered alone or as a pre-treatment, on the likelihood of relapse. Using these procedures, MOD administered 
alone, either acutely (Reichel and See, 2010; Holtz et al., 2012) or chronically (Reichel and See, 2012), did not reinstate behavior in rats initially trained to self-administer METH. Similar results were found with administration of R-MOD (Wang et al., 2015). However, in rhesus monkeys previously trained to self-administer cocaine, a high dose $(10 \mathrm{mg} / \mathrm{kg})$ induced reinstatement of cocaine responding (Andersen et al., 2010), which may indicate a species difference could be a factor in the obtained results. In METHprimed reinstatement tests, both acute (Reichel and See, 2010) and chronic (Reichel and See, 2012) MOD pretreatments attenuated reinstatement of drug-seeking behavior in both male and female rats (Holtz et al., 2012). MOD pretreatments did not significantly modify likelihood for reinstatement of cocaine self-administration behavior in rats (Deroche-Gamonet et al., 2002), but R-MOD reduced cocaine seeking at high doses (Zhang et al., 2017). Additionally, in rats, R-MOD pretreatment reduced nicotine-induced reinstatement of self-administration behavior (Wang et al., 2015). These results indicate that, in contrast to abused psychostimulants, MOD and R-MOD do not induce self-administration behavior, suggesting limited, if any, abuse liability. Also, they may diminish the potential for abuse of psychostimulants or reduce the drive to obtain them, and, finally, attenuate drug-induced reinstatement of drug seeking behaviors, suggesting a potential therapeutic effect in the prevention of relapse to drug use.

\section{Intracranial Self-Stimulation}

Intracranial self-stimulation is another indicator of the potential abuse liability of a substance. In this procedure, electrodes are placed in the medial forebrain bundle, and electrical stimulation is given when the subject performs the required operant task, for example nose-poking or pressing a lever. In comparison to self-administration studies, where the drug itself acts as the reinforcer, the electrical stimulation is the reinforcer in ICSS studies, allowing the assessment of whether the drug causes increased sensitivity to rewarding stimuli by altering the self-stimulation rates (Negus and Miller, 2014). Cocaine, METH, and other monoamine releasers have been found to facilitate ICSS (Bauer et al., 2013; Negus and Miller, 2014) with a correlation between facilitation rates and DA selectivity (Bauer et al., 2013; Negus and Miller, 2014), further implicating DA and DAT in the rewarding effects of these drugs.

Modafinil has been shown to facilitate ICSS responses in rats when administered orally (Lazenka and Negus, 2017) and intraperitoneally (Burrows et al., 2015). R-MOD shows a trend toward ICSS facilitation at high doses $(150 \mathrm{mg} / \mathrm{kg})$ in rats, without reaching significance (Burrows et al., 2015). However, when compared with commonly abused psychostimulants, such as methylphenidate or cocaine, MOD shows significant changes in ICSS rates only when administered at very high doses, while abused drugs show effects at significantly lower doses (Burrows et al., 2015; Lazenka and Negus, 2017). These dose differences may indicate that MOD abuse liability, if any, might require specific conditions, including very high doses, as compared to commonly abused psychostimulants. Indeed, MOD shows very low, if any, abuse liability in humans, and the benefits offered by MOD treatment against PSUD seem to outweigh the possibility of dependence.

\section{Drug Discrimination}

Administration of drugs, especially those abused by humans, would induce specific interoceptive stimuli that could be perceived and recognized by human subjects as well as animals (Kamien et al., 1993). The ability of subjects to discriminate between interoceptive stimuli elicited by a specific drug and those elicited by the drug's vehicle could be assessed in drug discrimination procedures (Porter et al., 2018). Indeed, the presence or absence of the drug stimulus could result in different operant responses, for example pressing a lever associated to the drug stimulus or that associated to the drug vehicle. Correct responses are usually rewarded with delivery of food pellets. After training with a specific drug, tests can be performed with administration of, for example, novel compounds. It is important to note that drugs belonging to the same pharmacological class (i.e., opioids, cannabinoids, psychostimulants) usually share a common discriminative stimulus specific for their drug class. Thus, while the drug-discrimination procedure does not measure the reinforcing/rewarding effects of drugs of abuse, similarities between subjective effects of a known abused psychostimulant and novel compounds might suggest their potential for abuse (Katz and Goldberg, 1988; Berquist and Fantegrossi, 2018). Thus, several drug-discrimination studies have tested the possibility that administration of MOD produced subjective effects similar to the discriminative stimulus effects of cocaine.

Modafinil doses below $100 \mathrm{mg} / \mathrm{kg}$ produced saline only responses when administered $30 \mathrm{~min}$ prior to testing, and higher doses partially substituted for cocaine in rats (Gold and Balster, 1996), but later studies found full cocaine substitution (Paterson et al., 2010). In Rhesus monkeys, MOD dose dependently substituted for cocaine in three of four animals at the highest doses when administered immediately prior to testing (Newman et al., 2010) and in mice, MOD fully substituted for cocaine (Loland et al., 2012; Mereu et al., 2017) when administered 5 or $60 \mathrm{~min}$ prior to testing. These results indicate that the subjective effects of MOD are similar to those of cocaine. However, there was a significant difference in potency for those effects, and MOD was found about 10 (Loland et al., 2012; Mereu et al., 2017) to 25 times less potent than cocaine (Gold and Balster, 1996). Further, MOD discrimination responses in rats were lower than that of ephedrine, a common over-the-counter decongestant and bronchodilator (Gold and Balster, 1996). These findings might indicate that high doses of MOD and R-MOD could have abuse potential, but the lower doses which would aid in reducing the likelihood of relapse have little abuse potential, as shown by lack of consistent reinforcing effects in the selfadministration studies above.

\section{Behavioral Tests Related to Cognitive Functions}

Cognitive impairments, such as memory deficits, decision making abilities, and learning rates are a potential concern as a consequence of persistent psychostimulant use (Block et al., 2002). While acute administration of psychostimulants has been 
found to positively affect cognitive functioning when given immediately prior to testing (Grilly, 2000; Del Olmo et al., 2007), long-term, repeated exposure to these drugs may produce detrimental cognitive effects. For example, in animal models, impairment of cognitive function has been reported in response to chronic administration of METH (Rogers et al., 2008) and cocaine (García-Pardo et al., 2017), among others (Marston et al., 1999; Dalley et al., 2005). MOD has been found to reverse some of the impairments induced by phencyclidine in rats (Redrobe et al., 2010), and by METH in rats and mice (González et al., 2014; Reichel et al., 2014). However, it has been reported that MOD administration had no effect on the object recognition of animals not pretreated with psychostimulants (Reichel et al., 2014), or spatial memory acquisition in rats not treated with DMSO (Shanmugasundaram et al., 2017), indicating a potential restoration of the cognitive impairments induced by drugs of abuse.

Modafinil has also been shown to improve decision making skills by decreasing impulsive responses in rats (Heyer-Osorno and Juárez, 2020), and both acute and chronic administration increases learning and memory abilities in mice (Béracochéa et al., 2002, 2003; Shuman et al., 2009) and rats (Ward et al., 2004; Morgan et al., 2007; Shuman et al., 2009). However, chronic MOD decreased long-term visuo-spatial memory errors, but increased operant conditioning learning errors, indicating an overall benefit for hippocampus dependent tasks in rats (Burgos et al., 2010). in a different study, an enhanced hippocampus dependent memory performance was reported after low doses of MOD $(0.75 \mathrm{mg} / \mathrm{kg})$, but not high doses $(75 \mathrm{mg} / \mathrm{kg})$, indicating a bell-shaped response curve (Shuman et al., 2009). Further, no effects on impulsive response rates were reported in healthy rats (Waters et al., 2005), however, these findings were explained later when improvements on a response rate task were only present in subjects previously showing slow or impaired response rates (Eagle et al., 2007). In general, these findings indicate that MOD has the potential to enhance cognitive abilities, especially when treating drug of abuse induced impairments, which may influence treatment engagement and likelihood of relapse in PSUD patients (Sofuoglu et al., 2013; Nuijten et al., 2016).

\section{HUMAN STUDIES ON MOD AS A POTENTIAL PHARMACOTHERAPY FOR PSUD}

Modafinil has shown therapeutic efficacy for treatment of individuals affected by narcolepsy and sleep disorders (Czeisler et al., 2005; Kumar, 2008), and its off-label uses have shown beneficial effects in improving cognitive function in patients with neuropsychiatric disorders, e.g., Parkinson's disease, ADHD or PSUD (Peñaloza et al., 2013; Turner et al., 2014). Even though MOD has been suggested as a potential therapeutic agent for the treatment of PSUD (Mereu et al., 2013; Tanda et al., 2021), initial concerns related to its potential abuse liability due to its effects on the central dopaminergic system, akin to those associated with many abused psychostimulants (Jasinski, 2000; Stoops et al., 2005; Volkow et al., 2009). Concerns about its potential for abuse have also been raised by the non-medical use of MOD by healthy individuals to enhance their cognitive function, attention, learning, and memory, in order to improve academic or work-related performance (Fond et al., 2016), leading to a significant debate about potential ethical issues related to a so called "cosmetic neurology" (Cakic, 2009; Sahakian and MoreinZamir, 2011). However, the increased non-medical use of MOD to potentially improve cognitive performance in school or work settings (Sharif et al., 2021) supports the very low risk, if any, of abuse liability (Kate et al., 2012; Ozturk and Deveci, 2014; Krishnan and Chary, 2015).

\section{Potential Therapeutic Effects of MOD for PSUD}

As summarized in Table 4, clinical studies testing MOD as a potential treatment for PSUD have generated different and sometime inconsistent results.

In an early double-blind, placebo-controlled 8-week study with 62 cocaine dependent patients, MOD $400 \mathrm{mg}$ daily, combined with CBT, significantly improved BE (benzoylecgonine - a cocaine metabolite) negative urine samples over placebo, and significantly increased abstinence rate (3 or more weeks) (Dackis et al., 2005). That study also indicated the safety of MOD administered to cocaine-dependent individuals (Dackis et al., 2005), a finding consistent with previous experimental safety studies that indicated the safety of the co-administration of MOD and intravenous cocaine (Dackis et al., 2003; Malcolm et al., 2006). More recently, another double-blind, placebocontrolled study with cocaine dependent patients $(N=94)$, over an 8-week period, showed that patients treated with $300 \mathrm{mg}$ MOD daily, combined with weekly individual therapy, were significantly more likely to be abstinent than those treated with placebo and weekly individual therapy (Kampman et al., 2015). Furthermore, MOD-treated patients reported significantly lower craving levels compared to those treated with placebo (Kampman et al., 2015). Other experimental human laboratory studies have investigated the potential role of MOD in modulating cocaine's subjective effects, such as self-reported decreases in 'good effects,' 'stimulation' and 'high' (Malcolm et al., 2006; Hart et al., 2008; McGaugh et al., 2009; Verrico et al., 2014). Further, a decrease in cocaine-associated cardiovascular effects was reported after treatments with both 200 and $400 \mathrm{mg}$ MOD doses, showing an objective physical response, as well as decreased self-administration of high cocaine doses (25 and $50 \mathrm{mg}$ ) (Hart et al., 2008).

While the safety of MOD treatments has also been observed in METH-dependent individuals (McGaugh et al., 2009), clinical studies on METH-dependent subjects are less promising than those in cocaine-dependents, although METH studies have been conducted in significantly smaller samples. For example, in a small trial of $13 \mathrm{METH}$-addicted patients treated with $200 \mathrm{mg}$ of MOD, the authors did not find any significant differences versus placebo, although they reported trends of lowering METH choice by $25 \%$ in 3 days of treatment (De La Garza et al., 2010). In a different study, MOD, $200 \mathrm{mg}$ daily, was tested over a 7day inpatient period on $19 \mathrm{METH}$ abstinent subjects, but no 
TABLE 4 | Results of clinical studies on MOD as a pharmacological therapy for PSUD, including studies on sleep disorders and cognitive dysfunction in PSUD patients.

\begin{tabular}{|c|c|c|c|c|c|}
\hline Dose/treatment & & & Experimental info & Main effect & References \\
\hline MOD 300 mg p.o. & Non-drug users & $\begin{array}{l}16 \text { healthy, non-drug } \\
\text { users }\end{array}$ & $\begin{array}{l}\text { Analysis of behavioral } \\
\text { and subjective effects } \\
\text { of MOD in comparison } \\
\text { to dextroamphetamine } \\
\text { and caffeine. }\end{array}$ & $\begin{array}{l}\text { MOD did not produce } \\
\text { subjective effects } \\
\text { associated with drug } \\
\text { abuse liability. }\end{array}$ & Warot et al. (1993) \\
\hline MOD 0-400 mg p.o. & & $\begin{array}{l}6 \text { healthy, non-drug } \\
\text { users }\end{array}$ & $\begin{array}{l}\text { Evaluation of } \\
\text { behavioral, and } \\
\text { subjective effects of } \\
\text { treatment }\end{array}$ & $\begin{array}{l}\text { Under active } \\
\text { conditions, MOD } \\
\text { produced reinforcing } \\
\text { effects. }\end{array}$ & Stoops et al. (2005) \\
\hline $\begin{array}{l}\text { MOD } 200-800 \text { mg, } \\
\text { methyl-phenidate } \\
45-90 \text { mg, or placebo p.o. }\end{array}$ & Poly-substances & $\begin{array}{l}24 \text { patients with } \\
\text { poly-substance abuse } \\
\text { history including } \\
\text { cocaine }\end{array}$ & $\begin{array}{l}\text { Evaluation of } \\
\text { behavioral, and } \\
\text { subjective effects of } \\
\text { treatment. }\end{array}$ & $\begin{array}{l}\text { MOD failed to produce } \\
\text { amphetamine-like } \\
\text { subjective effects. }\end{array}$ & Jasinski (2000) \\
\hline MOD 200-600 mg p.o. & Cocaine & $\begin{array}{l}9 \text { cocaine-dependent } \\
\text { patients }\end{array}$ & $\begin{array}{l}\text { Evaluation of the } \\
\text { subjective effects of } \\
\text { cocaine, MOD, and } \\
\text { placebo. }\end{array}$ & $\begin{array}{l}\text { MOD did not produce } \\
\text { cocaine-like subjective } \\
\text { effects. }\end{array}$ & Rush et al. (2002a) \\
\hline MOD 200-600 mg p.o. & & $\begin{array}{l}6 \text { cocaine-dependent } \\
\text { patients }\end{array}$ & $\begin{array}{l}\text { Evaluation of the } \\
\text { discriminative, } \\
\text { subjective, and } \\
\text { cardiovascular effects } \\
\text { of cocaine and MOD }\end{array}$ & $\begin{array}{l}\text { MOD did not produce } \\
\text { cocaine-like subjective } \\
\text { effects. }\end{array}$ & Rush et al. (2002b) \\
\hline $\begin{array}{l}\text { Placebo or MOD } 400 \text { mg } \\
\text { p.o. for } 8 \text { weeks with CBT }\end{array}$ & & $\begin{array}{l}62 \text { cocaine-dependent } \\
\text { patients }\end{array}$ & $\begin{array}{l}\text { Urine screen and } \\
\text { self-reporting to test } \\
\text { cocaine abstinence }\end{array}$ & $\begin{array}{l}\text { MOD (with CBT) } \\
\text { improved cocaine } \\
\text { dependence more than } \\
\text { placebo }\end{array}$ & Dackis et al. (2005) \\
\hline $\begin{array}{l}\text { MOD 0-800 mg p.o. in } \\
\text { combination with 0-40 mg } \\
\text { cocaine i.v. }\end{array}$ & & $\begin{array}{l}12 \text { cocaine-dependent } \\
\text { patients }\end{array}$ & $\begin{array}{l}\text { Analysis of interactions } \\
\text { between MOD and } \\
\text { cocaine }\end{array}$ & $\begin{array}{l}\text { MOD did not produce } \\
\text { any interactions with } \\
\text { cocaine }\end{array}$ & Malcolm et al. (2006) \\
\hline MOD 0-400 mg p.o. & & $\begin{array}{l}8 \text { cocaine-dependent } \\
\text { patients }\end{array}$ & $\begin{array}{l}\text { Analysis of } \\
\text { self-administration of } \\
\text { cocaine. }\end{array}$ & $\begin{array}{l}\text { MOD attenuated } \\
\text { self-administration of } \\
\text { cocaine. }\end{array}$ & Hart et al. (2008) \\
\hline $\begin{array}{l}\text { Placebo, MOD } 200 \text {, or } 400 \\
\text { mg p.o. for } 12 \text { weeks with } \\
\text { CBT }\end{array}$ & & $\begin{array}{l}210 \\
\text { cocaine-dependent } \\
\text { patients }\end{array}$ & $\begin{array}{l}\text { Urine screen and } \\
\text { self-reporting to test } \\
\text { cocaine abstinence }\end{array}$ & $\begin{array}{l}\text { CBT and MOD } \\
\text { effectively increased } \\
\text { cocaine non-use days }\end{array}$ & Anderson et al. (2009) \\
\hline $\begin{array}{l}\text { Placebo or MOD } 400 \text { mg } \\
\text { p.o. for } 16 \text { days. }\end{array}$ & & $\begin{array}{l}20 \text { cocaine-dependent } \\
\text { patients }\end{array}$ & Sleep analyses & $\begin{array}{l}\text { MOD normalized daily } \\
\text { sleep behavior and } \\
\text { architecture during } \\
\text { cocaine-abstinence }\end{array}$ & Morgan et al. (2010) \\
\hline $\begin{array}{l}\text { Placebo, MOD } 200 \text {, or } \\
400 \text { mg p.o. for } 8 \text { weeks } \\
\text { with CBT }\end{array}$ & & $\begin{array}{l}210 \\
\text { cocaine-dependent } \\
\text { patients }\end{array}$ & $\begin{array}{l}\text { Urine screen and } \\
\text { self-reporting to test } \\
\text { cocaine abstinence }\end{array}$ & $\begin{array}{l}\text { No significant effects on } \\
\text { abstinence for MOD } \\
\text { versus placebo, but } \\
\text { trends to significance in } \\
\text { male patients }\end{array}$ & Dackis et al. (2012) \\
\hline Placebo, or MOD 200 mg & & $\begin{array}{l}61 \text { cocaine-dependent } \\
\text { patients }\end{array}$ & $\begin{array}{l}\text { Urine screen to test } \\
\text { cocaine abstinence and } \\
\text { neurocognitive tests }\end{array}$ & $\begin{array}{l}\text { MOD improved working } \\
\text { memory performance }\end{array}$ & Kalechstein et al. (2013) \\
\hline $\begin{array}{l}\text { Placebo, MOD } 200 \text { mg, } \\
\text { escitalopram } 20 \text { mg, and } \\
\text { MOD } 200 \text { mg + } \\
\text { escitalopram } 20 \text { mg }\end{array}$ & & $\begin{array}{l}64 \text { cocaine-dependent } \\
\text { patients }\end{array}$ & $\begin{array}{l}\text { Testing of subjective } \\
\text { and reinforcing effects } \\
\text { as well as } \\
\text { cardiovascular } \\
\text { measures }\end{array}$ & $\begin{array}{l}\text { MOD blunted the } \\
\text { subjective effects of } \\
\text { cocaine but } \\
\text { escitalopram did not } \\
\text { enhance MOD effect }\end{array}$ & Verrico et al. (2014) \\
\hline Placebo or MOD 300 mg & & $\begin{array}{l}15 \text { cocaine-dependent } \\
\text { patients }\end{array}$ & $\begin{array}{l}\text { Response effort for } \\
\text { cocaine }\end{array}$ & $\begin{array}{l}\text { MOD significantly } \\
\text { decreased cocaine } \\
\text { choice but only under } \\
\text { high cost and } \\
\text { alternative condition }\end{array}$ & Foltin et al. (2016) \\
\hline $\begin{array}{l}\text { Placebo, MOD } 400 \text { mg, } \\
\text { and CBT }\end{array}$ & & $\begin{array}{l}57 \text { cocaine-dependent } \\
\text { patients }\end{array}$ & Sleep analyses & $\begin{array}{l}\text { MOD improved sleep } \\
\text { and decreased cocaine } \\
\text { use }\end{array}$ & Morgan et al. (2016) \\
\hline
\end{tabular}


TABLE 4 | Continued

\begin{tabular}{|c|c|c|c|c|c|}
\hline Dose/treatment & & & Experimental info & Main effect & References \\
\hline MOD 50-200 mg and CBT & METH & $\begin{array}{l}13 \mathrm{METH} \text {-dependent } \\
\text { patients }\end{array}$ & $\begin{array}{l}\text { Urine screen and } \\
\text { self-reporting to test } \\
\text { abstinence in HIV gay } \\
\text { men }\end{array}$ & $\begin{array}{l}\text { CBT and MOD showed } \\
\text { promise with high } \\
\text { treatment retention } \\
(77 \%) \text {. }\end{array}$ & McElhiney et al. (2009) \\
\hline MOD $400 \mathrm{mg}$ and CBT & & $\begin{array}{l}8 \mathrm{METH} \text {-dependent } \\
\text { patients }\end{array}$ & $\begin{array}{l}\text { Self-reporting of } \\
\text { psychological effects, } \\
\text { urine screen for drug } \\
\text { abstinence, and } \\
\text { cardiovascular effects }\end{array}$ & $\begin{array}{l}\text { No change in } \\
\text { cardiovascular or drug } \\
\text { abstinence effects }\end{array}$ & McGaugh et al. (2009) \\
\hline $\begin{array}{l}\text { Placebo or MOD } 200 \text { mg } \\
\text { p.o. for } 10 \text { weeks }\end{array}$ & & $\begin{array}{l}80 \text { METH-dependent } \\
\text { patients }\end{array}$ & $\begin{array}{l}\text { Urine screen and } \\
\text { self-reporting to test } \\
\text { METH abstinence, } \\
\text { blood pressure, weight } \\
\text { gain }\end{array}$ & $\begin{array}{l}\text { Reduction in systolic } \\
\text { blood pressure and } \\
\text { weight gain, but no } \\
\text { improvements on } \\
\text { METH dependence }\end{array}$ & Shearer et al. (2009) \\
\hline $\begin{array}{l}\text { Placebo or MOD } 200 \text { mg } \\
\text { p.o. }\end{array}$ & & $\begin{array}{l}13 \mathrm{METH} \text {-dependent } \\
\text { patients }\end{array}$ & $\begin{array}{l}\text { Subjective and } \\
\text { cardiovascular effects } \\
\text { of METH administration } \\
\text { and self-administration }\end{array}$ & $\begin{array}{l}\text { No statistical } \\
\text { differences in METH } \\
\text { use }\end{array}$ & De La Garza et al. (2010) \\
\hline $\begin{array}{l}\text { Placebo, MOD } 400 \text { mg } \\
\text { p.o., and CBT for } 12 \text { weeks }\end{array}$ & & $\begin{array}{l}71 \mathrm{METH} \text {-dependent } \\
\text { patients }\end{array}$ & $\begin{array}{l}\text { Urine screen to test } \\
\text { METH abstinence }\end{array}$ & $\begin{array}{l}\text { MOD did not improve } \\
\text { METH dependence } \\
\text { more than placebo }\end{array}$ & Heinzerling et al. (2010) \\
\hline $\begin{array}{l}\text { Placebo or MOD } 200 \text { mg } \\
\text { p.o. }\end{array}$ & & $\begin{array}{l}17 \mathrm{METH} \text {-dependent } \\
\text { patients }\end{array}$ & $\begin{array}{l}\text { Neuropsychological } \\
\text { tests }\end{array}$ & $\begin{array}{l}\text { MOD improved verbal } \\
\text { memory recall }\end{array}$ & Hester et al. (2010) \\
\hline $\begin{array}{l}400 \text { mg MOD or placebo } \\
\text { administered p.o. }\end{array}$ & & $\begin{array}{l}11 \mathrm{METH} \text {-dependent } \\
\text { patients }\end{array}$ & Memory tests & $\begin{array}{l}\text { MOD improved working } \\
\text { memory }\end{array}$ & Kalechstein et al. (2010) \\
\hline $\begin{array}{l}\text { Placebo or MOD } 200 \text { mg } \\
\text { p.o. }\end{array}$ & & $\begin{array}{l}24 \text { METH-dependent } \\
\text { patients and } 17 \text { healthy } \\
\text { subjects }\end{array}$ & $\begin{array}{l}\text { Cognitive tests } \\
\text { (inhibition, working } \\
\text { memory, attention) }\end{array}$ & $\begin{array}{l}\text { Improved attention in } \\
\text { patients with high } \\
\text { METH use }\end{array}$ & Ghahremani et al. (2011) \\
\hline $\begin{array}{l}\text { Placebo, MOD } 200 \text { mg, or } \\
400 \text { mg p.o. for } 12 \text { weeks }\end{array}$ & & $\begin{array}{l}210 \mathrm{METH} \text {-dependent } \\
\text { patients }\end{array}$ & $\begin{array}{l}\text { Urine drug screen for } \\
\text { METH non-use week }\end{array}$ & $\begin{array}{l}\text { No effects decrease in } \\
\text { METH use. Study } \\
\text { compounded by } \\
\text { patient compliance } \\
\text { issues. }\end{array}$ & Anderson et al. (2012) \\
\hline Placebo or MOD 200 mg & & $\begin{array}{l}18 \mathrm{METH} \text {-dependent } \\
\text { patients }\end{array}$ & $\begin{array}{l}\text { Evaluation of daytime } \\
\text { sleepiness and } \\
\text { abstinence }\end{array}$ & $\begin{array}{l}\text { Positive effects on } \\
\text { reducing daytime } \\
\text { sleepiness associated } \\
\text { with decreased METH } \\
\text { craving }\end{array}$ & Mahoney et al. (2012) \\
\hline $\begin{array}{l}\text { Placebo or MOD } 200 \text { mg } \\
\text { p.o. }\end{array}$ & & $\begin{array}{l}19 \mathrm{METH} \text {-dependent } \\
\text { patients }\end{array}$ & $\begin{array}{l}\text { Self-reporting of sleep, } \\
\text { physiological, and } \\
\text { craving/withdraw } \\
\text { measures }\end{array}$ & $\begin{array}{l}\text { No significant effects of } \\
\text { MOD. }\end{array}$ & Lee et al. (2013) \\
\hline MOD 200 mg p.o. & & $\begin{array}{l}80 \text { METH withdrawal } \\
\text { patients }\end{array}$ & Sleep analysis & $\begin{array}{l}\text { Improvements in sleep } \\
\text { quality }\end{array}$ & Moosavi et al. (2019) \\
\hline $\begin{array}{l}\text { Placebo or MOD } 200 \text { mg } \\
\text { p.o. }\end{array}$ & Nicotine & $\begin{array}{l}157 \\
\text { nicotine-dependent } \\
\text { patients }\end{array}$ & $\begin{array}{l}\text { Biochemical analysis of } \\
\text { nicotine abstinence }\end{array}$ & $\begin{array}{l}\text { No significant effects of } \\
\text { MOD }\end{array}$ & Schnoll et al. (2008) \\
\hline $\begin{array}{l}\text { Placebo, MOD } \\
200-800 \mathrm{mg} \text {, or } \\
\text { methylphenidate } 45-90 \mathrm{mg} \\
\text { p.o. }\end{array}$ & Poly-substance users & $\begin{array}{l}24 \text { patients with } \\
\text { polysubstance abuse } \\
\text { history including } \\
\text { cocaine }\end{array}$ & $\begin{array}{l}\text { Evaluation of } \\
\text { behavioral, and } \\
\text { subjective effects of } \\
\text { treatment }\end{array}$ & $\begin{array}{l}\text { MOD failed to produce } \\
\text { amphetamine like } \\
\text { subjective effects. }\end{array}$ & Jasinski (2000) \\
\hline
\end{tabular}

p.o., oral administration; i.v., intravenous; METH, methamphetamine; CBT, cognitive behavioral therapy.

differences, compared to placebo, were found for abstinence, reported craving, or sleep measures (Lee et al., 2013). In another study, METH-dependent patients were assigned to placebo or $200 \mathrm{mg}$ of MOD daily for 10 weeks (Shearer et al., 2009), resulting in no difference in retention and medical adherence between placebo and MOD in self-reported use and urine analysis. The study limitations of relying on self-reported measures and small sample size may confound the results and explain some of the variability among clinical studies for the efficacy of MOD in PSUD (Karila et al., 2010). 


\section{Efficacy of MOD Treatments in Subpopulations of Patients With Cocaine or METH Use Disorder but Without Comorbid Dependences From Other Substances}

With inconsistencies plaguing the results of MOD clinical studies, future research should be focused on the specific patient groups who showed beneficial effects from MOD treatment. While Shearer et al. (2009) didn't find significant differences between placebo and MOD treatments in the entire subjects sample, a post hoc analysis showed greatest reductions in METH use compared to placebo with MOD treatments in patients with only METH dependence (removing comorbid opioid dependent patients) (Shearer et al., 2009). MOD didn't increase the number of non-cocaine use days compared to placebo in a trial of cocaine-dependent patients, but post hoc analysis of data found that MOD was superior to placebo in patients without an alcohol codependency (Anderson et al., 2009). Similar outcomes were also reported in a more recent study, 8 -week double-blind, placebo-controlled clinical trial in cocainedependent subjects without comorbid alcohol dependence, where MOD (300 mg daily) treated patients were more likely to be abstinent from cocaine than patients treated with placebo (Kampman et al., 2015). In another trial on cocaine-dependent patients, no difference was reported between retention or abstinence for MOD treatment compared to placebo, but post hoc analysis revealed a significant gender difference with males taking $400 \mathrm{mg}$ MOD showing a greater estimate of abstinence (Dackis et al., 2012).

In a double-blind, placebo controlled trial, MOD, $400 \mathrm{mg}$ daily over 12 weeks, increased retention, while decreasing METH use, depression symptoms, and cravings in those with high METH use and low CBT attendance (Heinzerling et al., 2010). In a different study, it was found that escitalopram, a selective serotonin reuptake inhibitor and commonly prescribed antidepressant, decreased MOD's effects, raising concern of MOD's effect on populations of patients treated for depressive disorders alongside drug addiction (Verrico et al., 2014).

Reduced METH use in patients with an abuse diagnosis that included $\mathrm{HIV}+$ participants was found in a 12 -week $200 \mathrm{mg}$ MOD study (McElhiney et al., 2009). In the same study, the patients using METH on average 2.2 days per week reported better MOD effects on fatigue due to withdrawal, as well as maintaining abstinence, than patients who used meth 6 days per week (McElhiney et al., 2009). As clinical research on MOD continues, it is increasingly important to study the groups of individuals that do and do not respond to treatment in order to provide critical information toward precision medicine.

\section{MOD Effects on Sleep Disorders Related to Psychostimulant Use}

The relationship between sleep disorders and substance abuse is only loosely understood, but shows some relation with sleep problems reinforcing substance use disorders, as well as substance use leading to sleep disturbances (Angarita et al., 2016). In their review, Angarita et al. (2016) characterized several sleep disturbances produced by alcohol, cocaine, cannabis, and opioid short-term and long-term abstinence, suggesting that substantial research into the effectiveness of sleep agents for addiction treatment is needed. A more recent review links the effects of neurotransmitters on sleep during intoxication and withdrawal from a variety of drugs, but notes the lack of research depth on these neurological interactions and their bearing on drug abuse and dependence (Valentino and Volkow, 2020). Also, gender differences regarding the relationship between drug abstinence and sleep have been described (Coffey et al., 2000; Morgan et al., 2009). A study of short-term METH abstinence found a positive correlation between wanting a nap and craving METH (Mahoney et al., 2012). The study found that a single dose of MOD $200 \mathrm{mg}$ decreased daytime sleepiness, supporting the potential use of MOD as an adjunct treatment for PSUD.

Modafinil has been shown to increase and normalize slow wave sleep to healthy patterns in abstinent cocaine users (Morgan et al., 2010). It was also recently found that while increasing slow wave sleep did not lead to complete, continued abstinence, $400 \mathrm{mg}$ MOD treatment was associated with higher daily rates of abstinence and more consecutive days of abstinence (Morgan et al., 2016). Further, is has been reported that $200 \mathrm{mg}$ MOD improved the sleep quantity and pattern in patients during METH withdrawal (Moosavi et al., 2019).

\section{MOD Effects on Cognitive Impairment Produced by Psychostimulant Use}

Addiction brings changes to the brain beyond the reward pathway. Mental processing dysfunction can hamper rehabilitation attempts and, thus, a drug that can attenuate these risks would be beneficial to the addicted population (Gould, 2010). Cocaine-dependent patients in abstinence showed lower activation compared to healthy controls in areas associated with motor and cognitive functions (Kjome et al., 2010). There have been quite a few studies into MOD's effects on working memory. In a double-blind, placebo controlled study, it was shown that $400 \mathrm{mg}$ MOD improved working memory in $11 \mathrm{METH}$-dependent subjects, with poor performance at baseline, after 3 days of treatment (Kalechstein et al., 2010). The same group later showed, in a placebo controlled study, that MOD at $200 \mathrm{mg}$ improved visual and working memory in a group of 61 cocaine-dependent patients, as well as attention and impulsivity, with 5 days of treatment (Kalechstein et al., 2013). While promising, these studies also hold some limitations, in particular the short-term period of treatment and the small samples.

Even though not directly related to PSUD, effects of MOD on performance related to cognitive function were shown in a randomized, double-blind, placebo controlled, crossover study, where $200 \mathrm{mg}$ of MOD administered acutely improved cognitive control in alcohol-dependent patients, but not in the healthy control group (Schmaal et al., 2013). Also, the same researchers showed that administration of $200 \mathrm{mg}$ 
of MOD improved impulsive decision making in alcohol dependent patients compared to healthy controls (Schmaal et al., 2014). The alcohol dependent group had poor baseline performances compared to the healthy group. This difference could imply that MOD normalizes the brain's engagement to improve cognition to normal levels in lower performing groups, and the authors suggest that there was likely no room for improvement by MOD in the healthy controls (Schmaal et al., 2014). Further, it has been shown that MOD improved response inhibition in alcohol dependent patients whose initial response was poor (Schmaal et al., 2013). Similar effects related to PSUD were shown in METH-dependent patients in a double-blind, placebo controlled, crossover study, where $200 \mathrm{mg}$ of MOD increased poorly cognitive performance in METH-dependent patients to the same level as the healthy control (Ghahremani et al., 2011). Post hoc analysis also revealed that MOD produced larger effects in lower performing participants. Similar findings were also reported in METH-dependent patients where MOD treatments showed larger effects on inhibitory control, processing speed/attention, and motor speed in subject using higher levels of METH compared to those with lower METH usage (Dean et al., 2011). In another study, it was shown that cocaine dependent participants had lower Balloon Analog Risk Task (BART) scores but MOD treated cocaine-dependent participants had higher BART scores, which were comparable to the healthy placebo, showing a normalization of risk taking while on MOD (Canavan et al., 2014).

In a study combining MOD with CBT, it was found that crack cocaine-dependent patients with lower baselines of impulsivity (self-reported) had higher CBT retention and lower crack cocaine use (Nuijten et al., 2016). However, MOD treatment in these patients did not improve CBT retention or outcomes, which is likely as a result of the low MOD adherence during this trial. This study weakness was reported by the same researchers showing that only $10 \%$ adherence was reported during a 12 week CBT and MOD trial (Nuijten et al., 2015).

\section{BEYOND MOD: DRUG DEVELOPMENT OF MOD ANALOGS AS PHARMACOTHERAPEUTICS FOR PSUD}

The effectiveness of MOD as a medication for PSUD has been shown to reach significance in sub-populations of patients without comorbid dependencies from other drugs. In recent years, this important limitation of MOD efficacy has stimulated the development of new structural analogs of MOD to extend therapeutic actions to a broader population and, thus, maximize the effects of the parent drug for use in treatment of PSUD. Some of these novel agents showing atypical DAT blocker properties, have been highlighted in recently published reviews (Newman et al., 2021; Tanda et al., 2021). Among them, some have been shown to bind with high affinity to DAT, and those that promote an inward facing conformation of DAT have shown behavioral and neurochemical preclinical activities different from those of typical abused psychostimulants (Cao et al., 2010, 2016; OkunolaBakare et al., 2014). Such effects suggest an atypical DAT blocker profile (Keighron et al., 2019a; Newman et al., 2019, 2021; Tanda et al., 2021) and their potential as novel agents for use in the treatment of PSUD.

The effects of MOD analogs on DA neurochemistry have shown varying results (see Table 5). One of the tested analogs, JJC8-016, was unable to stimulate extracellular levels of DA after systemic administration (Zhang et al., 2017; Keighron et al., 2019b), in contrast to other MOD analogs, like JJC8-088 that significantly increased DA levels in a cocaine-like manner, or like JJC8-091 that elicited significant, but less efficacious, increases in DA levels. It is worth noting that the varying effects on stimulation of DA levels were not a result of an altered efficacy as DAT blockers. Indeed, all of these compounds were able to block and reduce DA uptake, an effect highly correlated to their affinity to DAT, as demonstrated by voltammetry studies in rats and mice (Keighron et al., 2019b; Newman et al., 2019). Moreover, their varying ability to enhance the stimulation of elicited DA release in voltammetry studies was unrelated to their affinity for DAT (Keighron et al., 2019b; Newman et al., 2019). These effects once more suggest that compounds that prefer or stabilize an inward facing conformation of DAT would produce limited, if any, cocaine-like effects (Keighron et al., 2019a,b; Giancola et al., 2020; Slack et al., 2020). The same MOD analogs have been tested in behavioral activities related to the reinforcing effects of psychostimulants, and those showing very low stimulation of DA output in microdialysis and voltammetry studies were also among those that produced limited stimulation of ambulatory activities (Keighron et al., 2019a,b; Giancola et al., 2020; Slack et al., 2020). Also, while they did not elicit acquisition or maintenance of self-administration behavior, these MOD analogs blunted cocaine or METH reinforcing and drug-seeking behaviors (Zhang et al., 2017; Tunstall et al., 2018; Newman et al., 2019), suggesting once more that their atypical DAT blocker profile and potential therapeutic activity could be useful as PSUD medications.

\section{CONCLUSION}

Modafinil is clinically approved for narcolepsy and other sleep disorders (Bastoji and Jouvet, 1988; Broughton et al., 1997; US Modafinil in Narcolepsy Multicenter Study Group, 1998, 2000), but its off-label use for treatment of several psychiatric disorders has been repeatedly reported (Ballon and Feifel, 2006; Peñaloza et al., 2013; Turner et al., 2014). During the last two decades, there have been several preclinical and clinical studies that suggested potential efficacy of MOD as a treatment for PSUD, but also contrasting results from other studies which limited its progression (Lee et al., 2013; Schmitz et al., 2014). Among the positive results, it is interesting to note that after many years of clinical use, there are only a few reports of abuse in MOD-treated patients (Kate et al., 2012; Ozturk and Deveci, 2014; Krishnan and Chary, 2015), a result in agreement with clinical and preclinical studies showing its 
TABLE 5 | Behavioral and neurochemical effects of MOD analogs.

\begin{tabular}{|c|c|c|c|c|}
\hline Agent(s) & Dose(s), species & Behavioral effects & Neurochemical effects & References \\
\hline \multirow[t]{7}{*}{ JJC8-016 } & $\begin{array}{l}\text { 10-30 mg/kg, i.p. } \\
\text { RAT }\end{array}$ & $\begin{array}{l}\text { NSE on locomotion when injected alone } \\
\downarrow \text { Cocaine-induced hyperlocomotion }\end{array}$ & NSE on stimulation of NAS DA & Zhang et al. (2017) \\
\hline & & $\begin{array}{l}\text { Did not induce self-administration } \\
\downarrow \text { Cocaine self-administration }\end{array}$ & & \\
\hline & & $\begin{array}{l}\downarrow \text { Reinstatement of cocaine seeking } \\
\text { behavior }\end{array}$ & & \\
\hline & $\begin{array}{l}\text { 3-100 mg/kg, i.p. } \\
\text { MICE }\end{array}$ & NSE on ambulatory behavior & NSE on stimulation of NAS DA & Keighron et al. (2019b) \\
\hline & & & $\begin{array}{l}\text { NSE on stimulation of evoked DA } \\
\text { release in NAS }\end{array}$ & \\
\hline & & & $\downarrow$ NAS DA clearance & \\
\hline & $\begin{array}{l}\text { 10-30 mg/kg, i.p. } \\
\text { RAT }\end{array}$ & $\begin{array}{l}\downarrow \text { METH self-administration following } \\
\text { both short and long access to drug }\end{array}$ & & Tunstall et al. (2018) \\
\hline \multirow[t]{7}{*}{ JJC8-088 } & $\begin{array}{l}1-56 \mathrm{mg} / \mathrm{kg}, \text { i.p. } \\
\text { RATS }\end{array}$ & $\downarrow$ Cocaine self-administration & $\uparrow$ NAS DA efflux & Newman et al. (2019) \\
\hline & & $\uparrow$ Optical intracranial self-stimulation & $\uparrow$ Evoked DA release in the NAS & \\
\hline & & NSE on cocaine PR breakpoints & $\downarrow$ NAS DA clearance & \\
\hline & $\begin{array}{l}\text { 3-30 mg/kg, i.p. } \\
\text { RAT }\end{array}$ & $\begin{array}{l}\text { NSE on METH self-administration } \\
\text { following both short and long access to } \\
\text { drug }\end{array}$ & & Tunstall et al. (2018) \\
\hline & $\begin{array}{l}\text { 3-56 mg/kg, i.p. } \\
\text { MICE }\end{array}$ & $\uparrow$ Ambulatory behavior & $\uparrow$ NAS DA efflux & Keighron et al. (2019b) \\
\hline & & & $\uparrow$ Evoked DA release in the NAS & \\
\hline & & & $\downarrow$ NAS DA clearance & \\
\hline \multirow[t]{8}{*}{ JJC8-091 } & $\begin{array}{l}1-56 \mathrm{mg} / \mathrm{kg} \text {, i.p. } \\
\text { RATS }\end{array}$ & NSE on cocaine FR self-administration & $\uparrow$ NAS DA efflux & Newman et al. (2019) \\
\hline & & $\downarrow P R$ breakpoints for cocaine & NSE on evoked NAS DA release & \\
\hline & & $\downarrow$ Cocaine primed reinstatement & $\downarrow$ NAS DA clearance & \\
\hline & & $\downarrow$ Optical intracranial self-stimulation & & \\
\hline & $\begin{array}{l}\text { 10-56 mg/kg, i.p. } \\
\text { RAT }\end{array}$ & $\begin{array}{l}\downarrow \text { METH self-administration following } \\
\text { both short and long access to drug }\end{array}$ & & Tunstall et al. (2018) \\
\hline & $\begin{array}{l}\text { 3-100 mg/kg, i.p. } \\
\text { MICE }\end{array}$ & NSE on ambulatory behavior & $\uparrow$ NAS DA efflux & Keighron et al. (2019b) \\
\hline & & & NSE on evoked NAS DA release & \\
\hline & & & $\downarrow$ NAS DA clearance & \\
\hline
\end{tabular}

NSE, not a significant effect; METH, methamphetamine; FR, fixed ratio; PR, progressive ratio; i.p., intraperitoneal; $\uparrow$, increase; $\downarrow$, decrease.

limited, if any, potential for abuse (Jasinski, 2000; DerocheGamonet et al., 2002; Myrick et al., 2004; Food and Drug Administration, 2007; Vosburg et al., 2010). On the other hand, disappointing results of clinical trials testing MOD as a treatment for PSUD have been obtained in the general population of drug-dependents. However, based on results from several of those reports, positive treatment outcomes have been found when the population sample included only subjects with psychostimulant dependency, without concurrent alcohol or other drug dependencies (Anderson et al., 2009; Shearer et al., 2009; Kampman et al., 2015). These studies underscore the importance of pursing personalized treatment approaches for PSUD, similarly to other medical disorders (Hamburg and Collins, 2010; Schork, 2015). It is clear that the complexity of PSUD, the huge differences in how PSUD develops among the population, and the presence of many other individual, genetic, or environmental variables, suggest it is unlikely that there will ever be a "silver bullet" medication to treat all individuals with PSUD. Thus, personalized medicine approaches, together with behavioral cognitive treatments, might be the most effective path to reduce the harm produced by PSUD. While MOD has been shown to improve several emerging pathological conditions related to psychostimulant use, i.e., dependence, sleep, and cognitive impairments, its overall limited success has triggered medicinal chemistry research toward discovery of structural analogs of MOD, that might hold more robust efficacy in PSUD. In conclusion, while MOD could be an effective pharmacological treatment already available for subpopulations of individuals suffering from PSUD, new pharmacological tools derived from MOD show promising preclinical efficacy and could help to provide more efficacious future treatment opportunities for PSUD.

\section{AUTHOR CONTRIBUTIONS}

All authors contributed to the manuscript and approved the submitted version. 


\section{FUNDING}

This work was supported by the Medication Development Program (Z1A-DA000611), National Institute on Drug Abuse, Intramural Research Program, NIH, DHHS.

\section{REFERENCES}

Alam, N., and Chaudhary, K. (2020). Repeated restraint stress potentiates methylphenidate and modafinil-induced behavioral sensitization in rats. Naunyn Schmiedebergs Arch. Pharmacol. 393, 785-795. doi: 10.1007/s00210019-01790-4

Alam, N., and Choudhary, K. (2018). Haloperidol attenuates Methylphenidate and Modafinil induced behavioural sensitization and cognitive enhancement. Metab. Brain Dis. 33, 893-906. doi: 10.1007/s11011-018-0190-x

Andersen, M. L., Kessler, E., Murnane, K. S., Mcclung, J. C., Tufik, S., and Howell, L. L. J. P. (2010). Dopamine transporter-related effects of modafinil in rhesus monkeys. Psychopharmacology 210, 439-448. doi: 10.1007/s00213-010-1839-2

Anderson, A. L., Li, S.-H., Biswas, K., Mcsherry, F., Holmes, T., Iturriaga, E., et al. (2012). Modafinil for the treatment of methamphetamine dependence. J. Drug Alcohol. Depend. 120, 135-141. doi: 10.1016/j.drugalcdep.2011.07.007

Anderson, A. L., Reid, M. S., Li, S.-H., Holmes, T., Shemanski, L., Slee, A., et al. (2009). Modafinil for the treatment of cocaine dependence. Drug Alcohol. Depend. 104, 133-139.

Angarita, G. A., Emadi, N., Hodges, S., and Morgan, P. T. (2016). Sleep abnormalities associated with alcohol, cannabis, cocaine, and opiate use: a comprehensive review. Addict. Sci. Clin. Pract. 11:9.

Arias-Carrión, O., Stamelou, M., Murillo-Rodríguez, E., Menéndez-González, M., and Pöppel, E. (2010). Dopaminergic reward system: a short integrative review. Int. Arch. Med. 3:24. doi: 10.1186/1755-7682-3-24

Ator, N. A., and Griffiths, R. R. (2003). Principles of drug abuse liability assessment in laboratory animals. Drug Alcohol. Depend. 70, S55-S72.

Ballon, J. S., and Feifel, D. (2006). A systematic review of modafinil: potential clinical uses and mechanisms of action. J. Clin. Psychiatry 67, 554-566. doi: 10.4088/jcp.v67n0406

Barr, A. M., Panenka, W. J., Macewan, G. W., Thornton, A. E., Lang, D. J., Honer, W. G., et al. (2006). The need for speed: an update on methamphetamine addiction. J. Psychiatry Neurosci. 31, 301-313.

Bastoji, H., and Jouvet, M. (1988). Successful treatment of idiopathic hypersomnia and narcolepsy with modafinil. Prog. Neuro Psychopharmacol. Biol. Psychiatry 12, 695-700. doi: 10.1016/0278-5846(88)90014-0

Bauer, C., Banks, M., Blough, B., and Negus, S. (2013). Use of intracranial selfstimulation to evaluate abuse-related and abuse-limiting effects of monoamine releasers in rats. Br. J. Pharmacol. 168, 850-862. doi: 10.1111/j.1476-5381.2012. 02214.x

Béracochéa, D., Cagnard, B., Célérier, A., Le Merrer, J., Pérès, M., and Piérard, C. (2001). First evidence of a delay-dependent working memory-enhancing effect of modafinil in mice. Neuroreport 12, 375-378. doi: 10.1097/00001756200102120-00038

Béracochéa, D., Celerier, A., Borde, N., Valleau, M., Peres, M., and Pierard, C. (2002). Improvement of learning processes following chronic systemic administration of modafinil in mice. Pharmacol. Biochem. Behav. 73, 723-728. doi: 10.1016/s0091-3057(02)00877-8

Béracochéa, D., Celerier, A., Peres, M., and Pierard, C. (2003). Enhancement of learning processes following an acute modafinil injection in mice. Pharmacol. Biochem. Behav. 76, 473-479. doi: 10.1016/j.pbb.2003. 09.007

Bernardi, R. E., Lewis, J. R., Lattal, K. M., and Berger, S. P. (2009). Modafinil reinstates a cocaine conditioned place preference following extinction in rats. Behav. Brain Res. 204, 250-253. doi: 10.1016/j.bbr.2009.05.028

Berquist, M. D., and Fantegrossi, W. E. (2018). Discriminative stimulus effects of psychostimulants. Curr. Top. Behav. Neurosci. 39, 29-49. doi: 10.1007/7854_ 2017_5

Beusterien, K. M., Rogers, A. E., Walsleben, J. A., Emsellem, H. A., Reblando, J. A., Wang, L., et al. (1999). Health-related quality of life effects of modafinil for treatment of narcolepsy. Sleep 22, 757-765. doi: 10.1093/sleep/22.6.757

\section{ACKNOWLEDGMENTS}

The authors would like to thank Dr. Gail Seabold for her suggestions and comments on an earlier version of this manuscript.

Block, R. I., Erwin, W. J., and Ghoneim, M. M. (2002). Chronic drug use and cognitive impairments. Pharmacol. Biochem. Behav. 73, 491-504. doi: 10.1016/ s0091-3057(02)00816-x

Bobak, M. J., Weber, M. W., Doellman, M. A., Schuweiler, D. R., Athens, J. M., Juliano, S. A., et al. (2016). Modafinil activates phasic dopamine signaling in dorsal and ventral striata. J. Pharmacol. Exp. Ther. 359, 460-470. doi: 10.1124/ jpet.116.236000

Bolla, K. I., Rothman, R., and Cadet, J. L. (1999). Dose-related neurobehavioral effects of chronic cocaine use. J. Neuropsychiatry Clin. Neurosci. 11, 361-369. doi: 10.1176/jnp.11.3.361

Boutrel, B., and Koob, G. F. (2004). What keeps us awake: the neuropharmacology of stimulants and wakefulness promoting medications. Sleep 27, 1181-1194. doi: 10.1093/sleep/27.6.1181

Boutrel, B., Steiner, N., and Halfon, O. (2013). The hypocretins and the reward function: what have we learned so far? Front. Behav. Neurosci. 7:59.

Bowers, M. S., Chen, B. T., and Bonci, A. (2010). AMPA receptor synaptic plasticity induced by psychostimulants: the past, present, and therapeutic future. Neuron 67, 11-24. doi: 10.1016/j.neuron.2010.06.004

Bradford, S. T., Stamatovic, S. M., Dondeti, R. S., Keep, R. F., and Andjelkovic, A. V. (2011). Nicotine aggravates the brain postischemic inflammatory response. Am. J. Physiol. Heart Circulatory Physiol. 300, H1518-H1529.

Brandao, W. N., Andersen, M. L., Palermo-Neto, J., Peron, J. P., and Zager, A. (2019). Therapeutic treatment with Modafinil decreases the severity of experimental autoimmune encephalomyelitis in mice. Int. Immunopharmacol. 75:105809. doi: 10.1016/j.intimp.2019.105809

Brandt, M. D., Ellwardt, E., and Storch, A. (2014). Short- and long-term treatment with modafinil differentially affects adult Hippocampal neurogenesis. Neuroscience 278, 267-275. doi: 10.1016/j.neuroscience.2014.08.014

Broughton, R., Fleming, J., George, C., Hill, J., Kryger, M., Moldofsky, H., et al. (1997). Randomized, double-blind, placebo-controlled crossover trial of modafinil in the treatment of excessive daytime sleepiness in narcolepsy. Neurology 49, 444-451. doi: 10.1212/wnl.49.2.444

Burgos, H., Castillo, A., Flores, O., Puentes, G., Morgan, C., Gatica, A., et al. (2010). Effect of modafinil on learning performance and neocortical long-term potentiation in rats. Brain Res. Bull. 83, 238-244. doi: 10.1016/j.brainresbull. 2010.08.010

Burrows, B. T., Watterson, L. R., Johnson, M. A., and Olive, M. F. (2015). Effects of modafinil and R-modafinil on brain stimulation reward thresholds: implications for their use in the treatment of psychostimulant dependence. J. Drug Alcohol. Res. 4:235958.

Cadet, J. L., Sheng, P., All, S., Rothman, R., Carlson, E., and Epstein, C. (1994). Rapid communication: attenuation of methamphetamine-induced neurotoxicity in Copper/Zinc Superoxide dismutase transgenic mice. J. Neurochem. 62, 380-383. doi: 10.1046/j.1471-4159.1994.62010380.x

Cakic, V. (2009). Smart drugs for cognitive enhancement: ethical and pragmatic considerations in the era of cosmetic neurology. J. Med. Ethics 35, 611-615. doi: 10.1136/jme.2009.030882

Calipari, E. S., Ferris, M. J., Salahpour, A., Caron, M. G., and Jones, S. R. (2013). Methylphenidate amplifies the potency and reinforcing effects of amphetamines by increasing dopamine transporter expression. Nat. Commun. 4:2720.

Canavan, S. V., Forselius, E. L., Bessette, A. J., and Morgan, P. T. (2014). Preliminary evidence for normalization of risk taking by modafinil in chronic cocaine users. Addict. Behav. 39, 1057-1061. doi: 10.1016/j.addbeh.2014.02.015

Cao, J., Prisinzano, T. E., Okunola, O. M., Kopajtic, T., Shook, M., Katz, J. L., et al. (2010). Structure-activity relationships at the monoamine transporters for a novel series of modafinil (2-[(diphenylmethyl)sulfinyl]acetamide) analogues. ACS Med. Chem. Lett. 2, 48-52.

Cao, J., Slack, R. D., Bakare, O. M., Burzynski, C., Rais, R., Slusher, B. S., et al. (2016). Novel and high Affinity 2-[(Diphenylmethyl)sulfinyl]acetamide 
(Modafinil) analogues as atypical dopamine transporter inhibitors. J. Med. Chem. 59, 10676-10691. doi: 10.1021/acs.jmedchem.6b01373

Chamberlain, S. R., and Robbins, T. W. (2013). Noradrenergic modulation of cognition: therapeutic implications. J. Psychopharmacol. 27, 694-718. doi: 10. $1177 / 0269881113480988$

Chang, S.-T., Tung, C.-S., Lin, Y.-L., Chuang, C.-H., Lee, A.-R., and Liu, Y.-P. (2010). Behavioral and cross sensitization after repeated exposure to modafinil and apomorphine in rats. Chin. J. Physiol. 53, 318-327. doi: 10.4077/cjp.2010. amk067

Chemelli, R. M., Willie, J. T., Sinton, C. M., Elmquist, J. K., Scammell, T., Lee, C., et al. (1999). Narcolepsy in orexin knockout mice: molecular genetics of sleep regulation. Cell 98, 437-451.

Clarke, P. B., and Pert, A. (1985). Autoradiographic evidence for nicotine receptors on nigrostriatal and mesolimbic dopaminergic neurons. Brain Res. 348, 355358. doi: 10.1016/0006-8993(85)90456-1

Coffey, S. F., Dansky, B. S., Carrigan, M. H., and Brady, K. T. (2000). Acute and protracted cocaine abstinence in an outpatient population: a prospective study of mood, sleep and withdrawal symptoms. Drug Alcohol. Depend. 59, 277-286. doi: 10.1016/s0376-8716(99)00126-x

Compton, W. M., Han, B., Blanco, C., Johnson, K., and Jones, C. M. (2018). Prevalence and correlates of prescription stimulant use, misuse, use disorders, and motivations for misuse among adults in the United States. Am. J. Psychiatry 175, 741-755. doi: 10.1176/appi.ajp.2018.17091048

Cope, Z. A., Minassian, A., Kreitner, D., Macqueen, D. A., Milienne-Petiot, M., Geyer, M. A., et al. (2017). Modafinil improves attentional performance in healthy, non-sleep deprived humans at doses not inducing hyperarousal across species. Neuropharmacology 125, 254-262. doi: 10.1016/j.neuropharm.2017.07. 031

Czeisler, C. A., Walsh, J. K., Roth, T., Hughes, R. J., Wright, K. P., Kingsbury, L., et al. (2005). Modafinil for excessive sleepiness associated with shift-work sleep disorder. N. Engl. J. Med. 353, 476-486. doi: 10.1056/nejmoa041292

Czub, S., Koutsilieri, E., Sopper, S., Czub, M., Stahl-Hennig, C., Müller, J., et al. (2001). Enhancement of central nervous system pathology in early simian immunodeficiency virus infection by dopaminergic drugs. Acta Neuropathol. $101,85-91$.

da Costa Soeiro, A., Moreira, K. D. M., Abrahao, K. P., Quadros, I. M. H., and Oliveira, M. G. M. (2012). Individual differences are critical in determining modafinil-induced behavioral sensitization and cross-sensitization with methamphetamine in mice. Behav. Brain Res. 233, 367-374. doi: 10.1016/ j.bbr.2012.05.023

Dackis, C. A., Kampman, K. M., Lynch, K. G., Pettinati, H. M., and O’brien, C. P. (2005). A double-blind, placebo-controlled trial of modafinil for cocaine dependence. Neuropsychopharmacology 30, 205-211. doi: 10.1038/sj.npp. 1300600

Dackis, C. A., Kampman, K. M., Lynch, K. G., Plebani, J. G., Pettinati, H. M., Sparkman, T., et al. (2012). A double-blind, placebo-controlled trial of modafinil for cocaine dependence. J. Subst. Abuse Treat. 43, 303-312.

Dackis, C. A., Lynch, K. G., Yu, E., Samaha, F. F., Kampman, K. M., Cornish, J. W., et al. (2003). Modafinil and cocaine: a double-blind, placebo-controlled drug interaction study. Drug Alcohol. Depend. 70, 29-37. doi: 10.1016/s03768716(02)00335-6

Dalley, J. W., Lääne, K., Pena, Y., Theobald, D. E., Everitt, B. J., and Robbins, T. W. (2005). Attentional and motivational deficits in rats withdrawn from intravenous self-administration of cocaine or heroin. Psychopharmacology 182, 579-587. doi: 10.1007/s00213-005-0107-3

Das, G. (1993). Cocaine abuse in North America: a milestone in history. J. Clin. Pharmacol. 33, 296-310. doi: 10.1002/j.1552-4604.1993.tb04661.x

Daws, L. C., Callaghan, P. D., Morón, J. A., Kahlig, K. M., Shippenberg, T. S., Javitch, J. A., et al. (2002). Cocaine increases dopamine uptake and cell surface expression of dopamine transporters. Biochem. Biophys. Res. Commun. 290, 1545-1550. doi: 10.1006/bbrc.2002.6384

De La Garza, R., Zorick, T., London, E. D., and Newton, T. F. (2010). Evaluation of modafinil effects on cardiovascular, subjective, and reinforcing effects of methamphetamine in methamphetamine-dependent volunteers. Drug Alcohol. Depend. 106, 173-180. doi: 10.1016/j.drugalcdep.2009.08.013

de Lima, M. S., Farrell, M., Reisser, A. A. L., and Soares, B. (2003). Antidepressants for cocaine dependence. Cochrane Database Syst. Res. 7:CD002950. de Saint Hilaire, Z., Orosco, M., Rouch, C., Blanc, G., and Nicolaidis, S. (2001). Variations in extracellular monoamines in the prefrontal cortex and medial hypothalamus after modafinil administration: a microdialysis study in rats. Neuroreport 12, 3533-3537. doi: 10.1097/00001756-200111160 $-00032$

Dean, A. C., Sevak, R. J., Monterosso, J. R., Hellemann, G., Sugar, C. A., and London, E. D. (2011). Acute modafinil effects on attention and inhibitory control in methamphetamine-dependent humans. J. Stud. Alcohol Drugs 72, 943-953. doi: 10.15288/jsad.2011.72.943

Del Olmo, N., Higuera-Matas, A., Miguens, M., Garcia-Lecumberri, C., and Ambrosio, E. (2007). Cocaine self-administration improves performance in a highly demanding water maze task. Psychopharmacology 195, 19-25. doi: 10.1007/s00213-007-0873-1

Deneau, G., Yanagita, T., and Seevers, M. (1969). Self-administration of psychoactive substances by the monkey. Psychopharmacologia 16, 30-48. doi: 10.1007/bf00405254

Deroche-Gamonet, V., Darnaudery, M., Bruins-Slot, L., Piat, F., Le Moal, M., and Piazza, P. (2002). Study of the addictive potential of modafinil in naive and cocaine-experienced rats. Psychopharmacology 161, 387-395. doi: 10.1007/ s00213-002-1080-8

Dhillon, N. K., Peng, F., Bokhari, S., Callen, S., Shin, S.-H., Zhu, X., et al. (2008). Cocaine-mediated alteration in tight junction protein expression and modulation of CCL2/CCR2 axis across the blood-brain barrier: implications for HIV-dementia. J. Neuroimmune Pharmacol. 3, 52-56. doi: 10.1007/s11481007-9091-1

Di Chiara, G., Acquas, E., Tanda, G., and Cadoni, C. (1993a). Drugs of abuse: biochemical surrogates of specific aspects of natural reward? Biochem. Soc. Symp. 59, 65-81.

Di Chiara, G., and Imperato, A. (1988). Drugs abused by humans preferentially increase synaptic dopamine concentrations in the mesolimbic system of freely moving rats. Proc. Natl. Acad. Sci. U.S.A. 85, 5274-5278. doi: 10.1073/pnas.85. 14.5274

Di Chiara, G., Tanda, G., Bassareo, V., Pontieri, F., Acquas, E., Fenu, S., et al. (1999). Drug addiction as a disorder of associative learning. Role of nucleus accumbens shell/extended amygdala dopamine. Ann. N. Y. Acad. Sci. 877, 461-485. doi: 10.1111/j.1749-6632.1999.tb09283.x

Di Chiara, G., Tanda, G., Cadoni, C., Acquas, E., Bassareo, V., and Carboni, E. (1998). Homologies and differences in the action of drugs of abuse and a conventional reinforcer (food) on dopamine transmission: an interpretative framework of the mechanism of drug dependence. Adv. Pharmacol. 42, 983987. doi: 10.1016/s1054-3589(08)60911-4

Di Chiara, G., Tanda, G., Frau, R., and Carboni, E. (1993b). On the preferential release of dopamine in the nucleus accumbens by amphetamine: further evidence obtained by vertically implanted concentric dialysis probes. Psychopharmacology 112, 398-402. doi: 10.1007/bf02244939

Diana, M. (2011). The dopamine hypothesis of drug addiction and its potential therapeutic value. Front. Psychiatry 2:64.

Duchêne, A., Perier, M., Zhao, Y., Liu, X., Thomasson, J., Chauveau, F., et al. (2016). Impact of astroglial connexins on modafinil pharmacological properties. Sleep 39, 1283-1292. doi: 10.5665/sleep.5854

Duflou, J. (2020). Psychostimulant use disorder and the heart. Addiction 115, 175-183. doi: 10.1111/add.14713

Eagle, D. M., Tufft, M. R., Goodchild, H. L., and Robbins, T. W. J. P. (2007). Differential effects of modafinil and methylphenidate on stop-signal reaction time task performance in the rat, and interactions with the dopamine receptor antagonist cis-flupenthixol. Psychopharmacology 192, 193-206. doi: 10.1007/ s00213-007-0701-7

Edwards, S., and Koob, G. F. (2013). Escalation of drug self-administration as a hallmark of persistent addiction liability. Behav. Pharmacol. 24, 356-362. doi: 10.1097/fbp.0b013e3283644d15

Elkashef, A., Holmes, T. H., Bloch, D. A., Shoptaw, S., Kampman, K., Reid, M. S., et al. (2005). Retrospective analyses of pooled data from CREST I and CREST II trials for treatment of cocaine dependence. Addiction 100, 91-101. doi: 10.1111/j.1360-0443.2005.00986.x

Espana, R., Baldo, B., Kelley, A., and Berridge, C. (2001). Wake-promoting and sleep-suppressing actions of hypocretin (orexin): basal forebrain sites of action. Neuroscience 106, 699-715. doi: 10.1016/s0306-4522(01)00319-0 
Farrell, M., Martin, N. K., Stockings, E., Bórquez, A., Cepeda, J. A., Degenhardt, L., et al. (2019). Responding to global stimulant use: challenges and opportunities. Lancet 394, 1652-1667. doi: 10.1016/s0140-6736(19)32230-5

Ferraro, L., Antonelli, T., O'connor, W. T., Tanganelli, S., Rambert, F. A., and Fuxe, K. (1997a). Modafinil: an antinarcoleptic drug with a different neurochemical profile to d-amphetamine and dopamine uptake blockers. Biol. Psychiatry 42, 1181-1183. doi: 10.1016/s0006-3223(97)00353-3

Ferraro, L., Antonelli, T., O'connor, W. T., Tanganelli, S., Rambert, F., and Fuxe, K. (1997b). The antinarcoleptic drug modafinil increases glutamate release in thalamic areas and hippocampus. Neuroreport 8, 2883-2887. doi: 10.1097/ 00001756-199709080-00016

Ferraro, L., Antonelli, T., O'connor, W. T., Tanganelli, S., Rambert, F. A., and Fuxe, K. (1998). The effects of modafinil on striatal, pallidal and nigral GABA and glutamate release in the conscious rat: evidence for a preferential inhibition of striato-pallidal GABA transmission. Neurosci. Lett. 253, 135-138.

Ferraro, L., Antonelli, T., Tanganelli, S., O'connor, W. T., De La Mora, M. P., Mendez-Franco, J., et al. (1999). The vigilance promoting drug modafinil increases extracellular glutamate levels in the medial preoptic area and the posterior hypothalamus of the conscious rat: prevention by local GABA A receptor blockade. Neuropsychopharmacology 20, 346-356. doi: 10.1016/s0893133x(98)00085-2

Ferraro, L., Fuxe, K., Tanganelli, S., Fernandez, M., Rambert, F., and Antonelli, T. (2000). Amplification of cortical serotonin release: a further neurochemical action of the vigilance-promoting drug modafinil. Neuropharmacology 39, 1974-1983. doi: 10.1016/s0028-3908(00)00019-8

Ferraro, L., O'connor, W. T., Li, X. M., Rimondini, R., Beani, L., Ungerstedt, U., et al. (1996a). Evidence for a differential cholecystokinin-B and -A receptor regulation of GABA release in the rat nucleus accumbens mediated via dopaminergic and cholinergic mechanisms. Neuroscience 73, 941-950. doi: 10.1016/0306-4522(96)00098-x

Ferraro, L., Tanganelli, S., O'connor, W. T., Antonelli, T., Rambert, F., and Fuxe, K. (1996b). The vigilance promoting drug modafinil decreases GABA release in the medial preoptic area and in the posterior hypothalamus of the awake rat: possible involvement of the serotonergic 5-HT3 receptor. Neurosci. Lett. 220, 5-8. doi: 10.1016/s0304-3940(96)13212-2

Ferraro, L., Tanganelli, S., O'connor, W. T., Antonelli, T., Rambert, F., and Fuxe, K. (1996c). The vigilance promoting drug modafinil increases dopamine release in the rat nucleus accumbens via the involvement of a local GABAergic mechanism. Eur. J. Pharmacol. 306, 33-39. doi: 10.1016/0014-2999(96)00182-3

Finke, K., Dodds, C. M., Bublak, P., Regenthal, R., Baumann, F., Manly, T., et al. (2010). Effects of modafinil and methylphenidate on visual attention capacity: a TVA-based study. Psychopharmacology 210, 317-329. doi: 10.1007/s00213010-1823-x

Foltin, R. W., Haney, M., Bedi, G., and Evans, S. M. (2016). Modafinil decreases cocaine choice in human cocaine smokers only when the response requirement and the alternative reinforcer magnitude are large. Pharmacol. Biochem. Behav. 150, 8-13. doi: 10.1016/j.pbb.2016.08.009

Fond, G., Gavaret, M., Vidal, C., Brunel, L., Riveline, J.-P., Micoulaud-Franchi, J.-A., et al. (2016). (Mis) use of prescribed stimulants in the medical student community: motives and behaviors: a population-based cross-sectional study. Medicine 95:e3366. doi: 10.1097/md.0000000000003366

Food and Drug Administration (2007). FDA Approved Labeling, PROVIGIL\$(modafinil) Tablets. Silver Spring: Food and Drug Administration.

Frye, M. A., Grunze, H., Suppes, T., Mcelroy, S. L., Keck, P. E., Walden, J., et al. (2007). A placebo-controlled evaluation of adjunctive modafinil in the treatment of bipolar depression. Am. J. Psychiatry 164, 1242-1249. doi: 10.1176/ appi.ajp.2007.06060981

Gan, X., Zhang, L., Berger, O., Stins, M. F., Way, D., Taub, D. D., et al. (1999). Cocaine enhances brain endothelial adhesion molecules and leukocyte migration. Clin. Immunol. 91, 68-76. doi: 10.1006/clim.1998.4683

Garcia, V. A., De Freitas, B. S., Busato, S. B., Portal, B. C. D. A., Piazza, F. C., and Schröder, N. J. N. (2013). Differential effects of modafinil on memory in naïve and memory-impaired rats. Neuropharmacology 75, 304-311. doi: 10.1016/j. neuropharm.2013.07.038

García-Pardo, M. P., De La Rubia, Ortí, J. E., and Calpe, M. A. A. (2017). Differential effects of MDMA and cocaine on inhibitory avoidance and object recognition tests in rodents. Neurobiol. Learn. Mem. 146, 1-11. doi: 10.1016/j. nlm.2017.10.013
Garcia-Rill, E., Heister, D. S., Ye, M., Charlesworth, A., and Hayar, A. (2007). Electrical coupling: novel mechanism for sleep-wake control. Sleep 30, 14051414. doi: $10.1093 /$ sleep/30.11.1405

Gawin, F. H. (1991). Cocaine addiction: psychology and neurophysiology. Science 251, 1580-1586. doi: 10.1126/science.2011738

Gerrard, P., and Malcolm, R. (2007). Mechanisms of modafinil: a review of current research. Neuropsychiatr. Dis. Treat. 3, 349-364.

Ghahremani, D. G., Tabibnia, G., Monterosso, J., Hellemann, G., Poldrack, R. A., and London, E. D. (2011). Effect of modafinil on learning and taskrelated brain activity in methamphetamine-dependent and healthy individuals. Neuropsychopharmacology 36, 950-959. doi: 10.1038/npp.2010.233

Ghasemzadeh, M. B., Vasudevan, P., and Mueller, C. (2009). Locomotor sensitization to cocaine is associated with distinct pattern of glutamate receptor trafficking to the postsynaptic density in prefrontal cortex: early versus late withdrawal effects. Pharmacol. Biochem. Behav. 92, 383-392. doi: 10.1016/j.pbb. 2008.12.004

Giancola, J. B., Bonifazi, A., Cao, J., Ku, T., Haraczy, A. J., Lam, J., et al. (2020). Structure-activity relationships for a series of (Bis(4fluorophenyl)methyl)sulfinylethyl-aminopiperidines and -piperidine amines at the dopamine transporter: Bioisosteric replacement of the piperazine improves metabolic stability. Eur. J. Med. Chem. 208:112674. doi: 10.1016/j.ejmech.2020.112674

Gold, L. H., and Balster, R. L. (1996). Evaluation of the cocaine-like discriminative stimulus effects and reinforcing effects of modafinil. Psychopharmacology 126, 286-292. doi: 10.1007/bf02247379

Golden, S. A., and Russo, S. J. (2012). Mechanisms of psychostimulant-induced structural plasticity. Cold Spring Harbor Perspect. Med. 2:a011957. doi: 10.1101/ cshperspect.a011957

Gonçalves, J., Baptista, S., Martins, T., Milhazes, N., Borges, F., Ribeiro, C. F., et al. (2010). Methamphetamine-induced neuroinflammation and neuronal dysfunction in the mice hippocampus: preventive effect of indomethacin. Eur. J. Neurosci. 31, 315-326. doi: 10.1111/j.1460-9568.2009.07059.x

González, B., Jayanthi, S., Gomez, N., Torres, O. V., Sosa, M. H., Bernardi, A., et al. (2018). Repeated methamphetamine and modafinil induce differential cognitive effects and specific histone acetylation and DNA methylation profiles in the mouse medial prefrontal cortex. Prog. Neuro Psychopharmacol. Biol. Psychiatry 82, 1-11. doi: 10.1016/j.pnpbp.2017.12.009

González, B., Raineri, M., Cadet, J. L., García-Rill, E., Urbano, F. J., and Bisagno, V. (2014). Modafinil improves methamphetamine-induced object recognition deficits and restores prefrontal cortex ERK signaling in mice. Neuropharmacology 87, 188-197. doi: 10.1016/j.neuropharm.2014.02.002

Gould, T. J. (2010). Addiction and cognition. Addict. Sci. Clin. Pract. 5:4.

Grilly, D. M. (2000). A verification of psychostimulant-induced improvement in sustained attention in rats: effects of d-amphetamine, nicotine, and pemoline. Exp. Clin. Psychopharmacol. 8, 14-21. doi: 10.1037/1064-1297.8.1.14

Haas, H. L., Sergeeva, O. A., and Selbach, O. (2008). Histamine in the nervous system. Physiol. Rev. 88, 1183-1241.

Hamburg, M. A., and Collins, F. S. (2010). The path to personalized medicine. N. Engl. J. Med. 363, 301-304.

Han, J., Chen, D., Liu, D., and Zhu, Y. (2018). Modafinil attenuates inflammation via inhibiting Akt/NF-kappaB pathway in apoE-deficient mouse model of atherosclerosis. Inflammopharmacology 26, 385-393. doi: 10.1007/s10787-0170387-3

Hart, C. L., Haney, M., Vosburg, S. K., Rubin, E., and Foltin, R. W. (2008). Smoked cocaine self-administration is decreased by modafinil. Neuropsychopharmacology 33, 761-768. doi: 10.1038/sj.npp.1301472

Hart, C., and Ksir, C. (1996). Nicotine effects on dopamine clearance in rat nucleus accumbens. J. Neurochem. 66, 216-221. doi: 10.1046/j.1471-4159.1996. 66010216.x

Hasler, B. P., Smith, L. J., Cousins, J. C., and Bootzin, R. R. (2012). Circadian rhythms, sleep, and substance abuse. Sleep Med. Rev. 16, 67-81. doi: 10.1016/j. smrv.2011.03.004

Hedegaard, H., Miniño, A. M., and Warner, M. (2020). Drug Overdose Deaths in the United States, 1999-2018: NCHS Data Brief No 356. Hyattsville, MD: National Center for Health Statistics. Available online at: https://www.cdc.gov/ nchs/products/databriefs/db356.htm

Heinzerling, K. G., Swanson, A.-N., Kim, S., Cederblom, L., Moe, A., Ling, W., et al. (2010). Randomized, double-blind, placebo-controlled trial of modafinil for the 
treatment of methamphetamine dependence. Drug Alcohol Depend. 109, 20-29. doi: 10.1016/j.drugalcdep.2009.11.023

Hermant, J.-F., Rambert, F. A., and Duteil, J. (1991). Awakening properties of modafinil: effect on nocturnal activity in monkeys (Macaca mulatta) after acute and repeated administration. Psychopharmacology 103, 28-32. doi: 10.1007/ bf02244069

Hester, R., Lee, N., Pennay, A., Nielsen, S., and Ferris, J. (2010). The effects of modafinil treatment on neuropsychological and attentional bias performance during 7-day inpatient withdrawal from methamphetamine dependence. Exp. Clin. Psychopharmacol. 18, 489-497. doi: 10.1037/a0021791

Heyer-Osorno, R., and Juárez, J. (2020). Modafinil reduces choice impulsivity while increasing motor activity in preadolescent rats treated prenatally with alcohol. Pharmacol. Biochem. Behav. 194:172936. doi: 10.1016/j.pbb.2020.172936

Holtz, N. A., Lozama, A., Prisinzano, T. E., and Carroll, M. E. (2012). Reinstatement of methamphetamine seeking in male and female rats treated with modafinil and allopregnanolone. Drug Alcohol Depend. 120, 233-237. doi: 10.1016/j.drugalcdep.2011.07.010

Howell, L. L., and Kimmel, H. L. (2008). Monoamine transporters and psychostimulant addiction. Biochem. Pharmacol. 75, 196-217. doi: 10.1016/j. bcp.2007.08.003

Huang, Z.-L., Qu, W.-M., Li, W.-D., Mochizuki, T., Eguchi, N., Watanabe, T., et al. (2001). Arousal effect of orexin a depends on activation of the histaminergic system. Proc. Natl. Acad. Sci. U.S.A. 98, 9965-9970. doi: 10.1073/ pnas. 181330998

Ishizuka, T., Murakami, M., and Yamatodani, A. (2008). Involvement of central histaminergic systems in modafinil-induced but not methylphenidate-induced increases in locomotor activity in rats. Eur. J. Pharmacol. 578, 209-215. doi: 10.1016/j.ejphar.2007.09.009

Ishizuka, T., Murotani, T., and Yamatodani, A. (2010). Modanifil activates the histaminergic system through the orexinergic neurons. Neurosci. Lett. 483, 193-196. doi: 10.1016/j.neulet.2010.08.005

Ishizuka, T., Sakamoto, Y., Sakurai, T., and Yamatodani, A. (2003). Modafinil increases histamine release in the anterior hypothalamus of rats. Neurosci. Lett. 339, 143-146. doi: 10.1016/s0304-3940(03)00006-5

Ishizuka, T., Yamamoto, Y., and Yamatodani, A. (2002). The effect of orexin-a and$\mathrm{B}$ on the histamine release in the anterior hypothalamus in rats. Neurosci. Lett. 323, 93-96. doi: 10.1016/s0304-3940(01)02552-6

Itzhak, Y., and Martin, J. L. (2002). Cocaine-induced conditioned place preference in mice: induction, extinction and reinstatement by related psychostimulants. Neuropsychopharmacology 26, 130-134. doi: 10.1016/s0893-133x(01)00303-7

Jasinski, D. R. (2000). An evaluation of the abuse potential of modafinil using methylphenidate as a reference. J. Psychopharmacol. 14, 53-60. doi: 10.1177/ 026988110001400107

Jones, B. E., Harper, S. T., and Halaris, A. E. (1977). Effects of locus coeruleus lesions upon cerebral monoamine content, sleep-wakefulness states and the response to amphetamine in the cat. Brain Res. 124, 473-496. doi: 10.1016/ 0006-8993(77)90948-9

Jordan, C. J., Cao, J., Newman, A. H., and Xi, Z. X. (2019). Progress in agonist therapy for substance use disorders: lessons learned from methadone and buprenorphine. Neuropharmacology 158:107609. doi: 10.1016/j.neuropharm. 2019.04.015

Kahlig, K. M., and Galli, A. (2003). Regulation of dopamine transporter function and plasma membrane expression by dopamine, amphetamine, and cocaine. Eur. J. Pharmacol. 479, 153-158. doi: 10.1016/j.ejphar.2003.08.065

Kahlig, K. M., Lute, B. J., Wei, Y., Loland, C. J., Gether, U., Javitch, J. A., et al. (2006). Regulation of dopamine transporter trafficking by intracellular amphetamine. Mol. Pharmacol. 70, 542-548. doi: 10.1124/mol.106.023952

Kalechstein, A. D., De La Garza, I., Richard, and Newton, T. F. (2010). Modafinil administration improves working memory in methamphetaminedependent individuals who demonstrate baseline impairment. Am. J. Addict. 19, 340-344.

Kalechstein, A., Mahoney, J., Yoon, J., Bennett, R., and De La Garza Ii, R. (2013). Modafinil, but not escitalopram, improves working memory and sustained attention in long-term, high-dose cocaine users. Neuropharmacology 64, 472478. doi: 10.1016/j.neuropharm.2012.06.064

Kalivas, P. W. (2009). The glutamate homeostasis hypothesis of addiction. Nat. Rev. Neurosci. 10, 561-572. doi: 10.1038/nrn2515
Kalivas, P. W., and Duffy, P. (1993). Time course of extracellular dopamine and behavioral sensitization to cocaine. I. dopamine axon terminals. J. Neurosci.. 13, 266-275. doi: 10.1523/jneurosci.13-01-00266.1993

Kamien, J. B., Bickel, W. K., Hughes, J. R., Higgins, S. T., and Smith, B. J. (1993). Drug discrimination by humans compared to nonhumans: current status and future directions. Psychopharmacology 111, 259-270. doi: 10.1007/ bf02244940

Kampman, K. M., Lynch, K. G., Pettinati, H. M., Spratt, K., Wierzbicki, M. R., Dackis, C., et al. (2015). A double blind, placebo controlled trial of modafinil for the treatment of cocaine dependence without co-morbid alcohol dependence. Drug Alcohol Depend. 155, 105-110. doi: 10.1016/j.drugalcdep.2015.08.005

Karila, L., Weinstein, A., Aubin, H. J., Benyamina, A., Reynaud, M., and Batki, S. L. (2010). Pharmacological approaches to methamphetamine dependence: a focused review. Br. J. Clin. Pharmacol. 69, 578-592. doi: 10.1111/j.1365-2125. 2010.03639.x

Kate, N., Grover, S., and Ghormode, D. (2012). Dependence on supratherapeutic doses of modafinil: a case report. Prim. Care Companion CNS Disord. 14:CC.11101333.

Katz, J. L., and Goldberg, S. R. (1988). Preclinical assessment of abuse liability of drugs. Agents Actions 23, 18-26. doi: 10.1007/bf01967174

Kauer, J. A., and Malenka, R. C. (2007). Synaptic plasticity and addiction. Nat. Rev. Neurosci. 8, 844-858.

Kaye, S., Mcketin, R., Duflou, J., and Darke, S. (2007). Methamphetamine and cardiovascular pathology: a review of the evidence. Addiction 102, 1204-1211. doi: 10.1111/j.1360-0443.2007.01874.x

Keighron, J. D., Giancola, J. B., Shaffer, R. J., Demarco, E. M., Coggiano, M. A., Slack, R. D., et al. (2019a). Distinct effects of (R)-modafinil and its (R)-and (S)fluoro-analogs on mesolimbic extracellular dopamine assessed by voltammetry and microdialysis in rats. Eur. J. Neurosci. 50, 2045-2053. doi: 10.1111/ejn. 14256

Keighron, J. D., Quarterman, J. C., Cao, J., Demarco, E. M., Coggiano, M. A., Gleaves, A., et al. (2019b). Effects of (R)-modafinil and modafinil analogues on dopamine dynamics assessed by voltammetry and microdialysis in the mouse nucleus accumbens shell. ACS Chem. Neurosci. 10, 2012-2021. doi: 10.1021/ acschemneuro.8b00340

Killgore, W. D., Kahn-Greene, E. T., Grugle, N. L., Killgore, D. B., and Balkin, T. J. (2009). Sustaining executive functions during sleep deprivation: a comparison of caffeine, dextroamphetamine, and modafinil. Sleep 32, 205-216. doi: 10. 1093/sleep/32.2.205

Kjome, K. L., Lane, S. D., Schmitz, J. M., Green, C., Ma, L., Prasla, I., et al. (2010). Relationship between impulsivity and decision making in cocaine dependence. Psychiatry Res. 178, 299-304. doi: 10.1016/j.psychres.2009.11.024

Kohut, S. J., Hiranita, T., Hong, S.-K., Ebbs, A. L., Tronci, V., Green, J., et al. (2014). Preference for distinct functional conformations of the dopamine transporter alters the relationship between subjective effects of cocaine and stimulation of mesolimbic dopamine. Biol. Psychiatry 76, 802-809. doi: 10.1016/j.biopsych. 2014.03.031

Koob, G. F. (1992). Dopamine, addiction and reward. Semin. Neurosci. 4, 139-148. doi: 10.1016/1044-5765(92)90012-q

Koob, G. F., Sanna, P. P., and Bloom, F. E. (1998). Neuroscience of addiction. Neuron 21, 467-476.

Kousik, S. M., Napier, T. C., and Carvey, P. M. (2012). The effects of psychostimulant drugs on blood brain barrier function and neuroinflammation. Front. Pharmacol. 3:121.

Krishnan, R., and Chary, K. V. (2015). A rare case modafinil dependence. J. Pharmacol. Pharmacother. 6, 49-50. doi: 10.4103/0976-500x.149149

Kuhar, M., Ritz, M., and Boja, J. (1991). The dopamine hypothesis of the reinforcing properties of cocaine. Trends Neurosci. 14, 299-302. doi: 10.1016/01662236(91)90141-g

Kumar, R. (2008). Approved and investigational uses of modafinil. Drugs 68, 1803-1839. doi: 10.2165/00003495-200868130-00003

Lai, Y.-T., Tsai, Y.-P. N., Cherng, C. G., Ke, J.-J., Ho, M.-C., Tsai, C.-W., et al. (2009). Lipopolysaccharide mitagates methamphetamine-induced striatal dopamine depletion via modulating local TNF- $\alpha$ and dopamine transporter expression. J. Neural. Transm. 116, 405-415. doi: 10.1007/s00702-009-0204-2

Lange, R. A., and Hillis, L. D. (2001). Cardiovascular complications of cocaine use. N. Engl. J. Med. 345, 351-358. 
Laviolette, S. R., and Van Der Kooy, D. (2004). The neurobiology of nicotine addiction: bridging the gap from molecules to behaviour. Nat. Rev. Neurosci. 5, 55-65. doi: 10.1038/nrn1298

Lazenka, M. F., and Negus, S. S. (2017). Oral modafinil facilitates intracranial self-stimulation in rats: comparison to methylphenidate. Behav. Pharmacol. 28, 318-322. doi: $10.1097 / \mathrm{fbp} .0000000000000288$

Lee, N., Pennay, A., Hester, R., Mcketin, R., Nielsen, S., and Ferris, J. (2013). A pilot randomised controlled trial of modafinil during acute methamphetamine withdrawal: feasibility, tolerability and clinical outcomes. Drug Alcohol Rev. 32, 88-95. doi: 10.1111/j.1465-3362.2012.00473.x

Lee, Y. W., Hennig, B., Fiala, M., Kim, K. S., and Toborek, M. (2001). Cocaine activates redox-regulated transcription factors and induces TNF- $\alpha$ expression in human brain endothelial cells. Brain Res. 920, 125-133. doi: 10.1016/s00068993(01)03047-5

Little, K. Y., Elmer, L. W., Zhong, H., Scheys, J. O., and Zhang, L. (2002). Cocaine induction of dopamine transporter trafficking to the plasma membrane. Mol. Pharmacol. 61, 436-445. doi: 10.1124/mol.61.2.436

Liu, J., Wu, R., and Li, J.-X. (2020). TAAR1 and psychostimulant addiction. Cell Mol. Neurobiol. 40, 229-238. doi: 10.1007/s10571-020-00792-8

Liu, X., Petit, J.-M., Ezan, P., Gyger, J., Magistretti, P., and Giaume, C. (2013). The psychostimulant modafinil enhances gap junctional communication in cortical astrocytes. Neuropharmacology 75, 533-538. doi: 10.1016/j.neuropharm.2013. 04.019

Liu, Y., Le Foll, B., Liu, Y., Wang, X., and Lu, L. (2008). Conditioned place preference induced by licit drugs: establishment, extinction, and reinstatement. Sci. World J. 8, 1228-1245. doi: 10.1100/tsw.2008.154

Loland, C. J., Mereu, M., Okunola, O. M., Cao, J., Prisinzano, T. E., Mazier, S., et al. (2012). R-modafinil (armodafinil): a unique dopamine uptake inhibitor and potential medication for psychostimulant abuse. Biol. Psychiatry 72, 405-413. doi: 10.1016/j.biopsych.2012.03.022

Luscher, C., and Malenka, R. C. (2011). Drug-evoked synaptic plasticity in addiction: from molecular changes to circuit remodeling. Neuron 69, 650-663. doi: 10.1016/j.neuron.2011.01.017

Madras, B. K., Xie, Z., Lin, Z., Jassen, A., Panas, H., Lynch, L., et al. (2006). Modafinil occupies dopamine and norepinephrine transporters in vivo and modulates the transporters and trace amine activity in vitro. J. Pharmacol. Exp. Ther. 319, 561-569. doi: 10.1124/jpet.106.106583

Mahfoud, Y., Talih, F., Streem, D., and Budur, K. (2009). Sleep disorders in substance abusers: how common are they? Psychiatry 6, 38-42.

Mahler, S. V., Hensley-Simon, M., Tahsili-Fahadan, P., Lalumiere, R. T., Thomas, C., Fallon, R. V., et al. (2014). Modafinil attenuates reinstatement of cocaine seeking: role for cystine-glutamate exchange and metabotropic glutamate receptors. Addict. Biol. 19, 49-60. doi: 10.1111/j.1369-1600.2012.00506.x

Mahoney, J. J., Jackson, B. J., Kalechstein, A. D., De La Garza, R., Chang, L. C., and Newton, T. F. (2012). Acute modafinil exposure reduces daytime sleepiness in abstinent methamphetamine-dependent volunteers. Int. J. Neuropsychopharmacol. 15, 1241-1249. doi: 10.1017/s1461145711001805

Makris, A. P., Rush, C. R., Frederich, R. C., and Kelly, T. H. (2004). Wakepromoting agents with different mechanisms of action: comparison of effects of modafinil and amphetamine on food intake and cardiovascular activity. Appetite 42, 185-195. doi: 10.1016/j.appet.2003.11.003

Malcolm, R., Swayngim, K., Donovan, J. L., Devane, C. L., Elkashef, A., Chiang, N., et al. (2006). Modafinil and cocaine interactions. Am. J. Drug Alcohol Abuse 32, 577-587. doi: 10.1016/b978-0-12-803750-8.00058-0

Marston, H. M., Reid, M. E., Lawrence, J. A., Olverman, H. J., and Butcher, S. P. (1999). Behavioural analysis of the acute and chronic effects of MDMA treatment in the rat. Psychopharmacology 144, 67-76. doi: 10.1007/ s002130050978

McElhiney, M. C., Rabkin, J. G., Rabkin, R., and Nunes, E. V. (2009). Provigil (modafinil) plus cognitive behavioral therapy for methamphetamine use in HIV+ gay men: a pilot study. Am. J. Drug Alcohol Abuse 35, 34-37. doi: 10.1080/00952990802342907

McGaugh, J., Mancino, M. J., Feldman, Z., Chopra, M. P., Gentry, W. B., Cargile, C., et al. (2009). Open label pilot study of modafinil for methamphetamine dependence. J. Clin. Psychopharmacol. 29, 488-491. doi: 10.1097/jcp. 0b013e3181b591e0

Meldrum, B. S. (2000). Glutamate as a neurotransmitter in the brain: review of physiology and pathology. J. Nutr. 130, 1007S-1015S.
Mereu, M., Bonci, A., Newman, A. H., and Tanda, G. (2013). The neurobiology of modafinil as an enhancer of cognitive performance and a potential treatment for substance use disorders. Psychopharmacology 229, 415-434. doi: 10.1007/ s00213-013-3232-4

Mereu, M., Chun, L. E., Prisinzano, T. E., Newman, A. H., Katz, J. L., and Tanda, G. (2017). The unique psychostimulant profile of $( \pm)$-modafinil: investigation of behavioral and neurochemical effects in mice. Eur. J. Neurosci. 45, 167-174. doi: 10.1111/ejn.13376

Mereu, M., Hiranita, T., Jordan, C. J., Chun, L. E., Lopez, J. P., Coggiano, M. A., et al. (2020). Modafinil potentiates cocaine self-administration by a dopamine-independent mechanism: possible involvement of gap junctions. Neuropsychopharmacology 45, 1518-1526. doi: 10.1038/s41386-0200680-5

Mereu, M., Tronci, V., Chun, L. E., Thomas, A. M., Green, J. L., Katz, J. L., et al. (2015). Cocaine-induced endocannabinoid release modulates behavioral and neurochemical sensitization in mice. Addict. Biol. 20, 91-103. doi: 10.1111/adb. 12080

Mignot, E., Nishino, S., Guilleminault, C., and Dement, W. (1994). Modafinil binds to the dopamine uptake carrier site with low affinity. Sleep 17, 436-437. doi: 10.1093/sleep/17.5.436

Minzenberg, M. J., and Carter, C. S. (2008). Modafinil: a review of neurochemical actions and effects on cognition. Neuropsychopharmacology 33, 1477-1502. doi: 10.1038/sj.npp.1301534

Mitchell, H. A., and Weinshenker, D. (2010). Good night and good luck: norepinephrine in sleep pharmacology. Biochem. Pharmacol. 79, 801-809. doi: 10.1016/j.bcp.2009.10.004

Monti, J. M. (2013). The neurotransmitters of sleep and wake, a physiological reviews series. Sleep Med. Rev. 17, 313-315. doi: 10.1016/j.smrv.2013. 02.004

Moosavi, S. M., Yazdani-Charati, J., and Amini, F. (2019). Effects of modafinil on sleep pattern during methamphetamine withdrawal: a double-blind randomized controlled trial. Addict. Health 11, 165-172.

Morgan, P. T., Angarita, G. A., Canavan, S., Pittman, B., Oberleitner, L., Malison, R. T., et al. (2016). Modafinil and sleep architecture in an inpatient-outpatient treatment study of cocaine dependence. Drug Alcohol Depend. 160, 49-56. doi: 10.1016/j.drugalcdep.2015.12.004

Morgan, P. T., Pace-Schott, E., Pittman, B., Stickgold, R., and Malison, R. T. (2010). Normalizing effects of modafinil on sleep in chronic cocaine users. Am. J. Psychiatry 167, 331-340. doi: 10.1176/appi.ajp.2009.09050613

Morgan, P. T., Paliwal, P., Malison, R. T., and Sinha, R. (2009). Sex differences in sleep and sleep-dependent learning in abstinent cocaine users. Pharmacol. Biochem. Behav. 93, 54-58. doi: 10.1016/j.pbb.2009.04.006

Morgan, R. E., Crowley, J. M., Smith, R. H., Laroche, R. B., and Dopheide, M. M. (2007). Modafinil improves attention, inhibitory control, and reaction time in healthy, middle-aged rats. Pharmacol. Biochem. Behav. 86, 531-541. doi: 10.1016/j.pbb.2007.01.015

Mortensen, O. V., and Amara, S. G. (2003). Dynamic regulation of the dopamine transporter. Eur. J. Pharmacol 479, 159-170.

Mueller, D., and Stewart, J. (2000). Cocaine-induced conditioned place preference: reinstatement by priming injections of cocaine after extinction. Behav. Brain Res. 115, 39-47. doi: 10.1016/s0166-4328(00)00239-4

Müller, U., Steffenhagen, N., Regenthal, R., and Bublak, P. (2004). Effects of modafinil on working memory processes in humans. Psychopharmacology 177, 161-169. doi: 10.1007/s00213-004-1926-3

Munzar, P., Tanda, G., Justinova, Z., and Goldberg, S. R. (2004). Histamine h3 receptor antagonists potentiate methamphetamine selfadministration and methamphetamine-induced accumbal dopamine release. Neuropsychopharmacology 29, 705-717. doi: 10.1038/sj.npp.1300380

Murillo-Rodríguez, E., Haro, R., Palomero-Rivero, M., Millán-Aldaco, D., and Drucker-Colín, R. (2007). Modafinil enhances extracellular levels of dopamine in the nucleus accumbens and increases wakefulness in rats. Behav. Brain Res. 176, 353-357. doi: 10.1016/j.bbr.2006.10.016

Myrick, H., Malcolm, R., Taylor, B., and Larowe, S. (2004). Modafinil: preclinical, clinical, and post-marketing surveillance-a review of abuse liability issues. Ann. Clin. Psychiatry 16, 101-109. doi: 10.1080/10401230490453743

Napier, T. C., Herrold, A. A., and De Wit, H. (2013). Using conditioned place preference to identify relapse prevention medications. Neurosci. Biobehav. Rev. 37, 2081-2086. doi: 10.1016/j.neubiorev.2013.05.002 
Nath, A., Hauser, K. F., Wojna, V., Booze, R. M., Maragos, W., Prendergast, M., et al. (2002). Molecular basis for interactions of HIV and drugs of abuse. J. Acquir. Immune Defic. Syndr. 31, S62-S69.

Negus, S. S., and Miller, L. L. (2014). Intracranial self-stimulation to evaluate abuse potential of drugs. Pharmacol. Rev. 66, 869-917. doi: 10.1124/pr.112.007419

Nestler, E. J. (2005). The neurobiology of cocaine addiction. Sci. Pract. Perspect. 3 , $4-10$.

Newman, A. H., Cao, J., Keighron, J. D., Jordan, C. J., Bi, G.-H., Liang, Y., et al. (2019). Translating the atypical dopamine uptake inhibitor hypothesis toward therapeutics for treatment of psychostimulant use disorders. Neuropsychopharmacology 44, 1435-1444. doi: 10.1038/s41386-019-0366-Z

Newman, A. H., Ku, T., Jordan, C. J., Bonifazi, A., and Xi, Z. X. (2021). New drugs, old targets: tweaking the dopamine system to treat psychostimulant use disorders. Annu. Rev. Pharmacol. Toxicol. 61, 609-628. doi: 10.1146/annurevpharmtox-030220- 124205

Newman, J. L., Negus, S. S., Lozama, A., Prisinzano, T. E., and Mello, N. K. (2010). Behavioral evaluation of modafinil and the abuse-related effects of cocaine in rhesus monkeys. Exp. Clin. Psychopharmacol. 18, 395-408. doi: 10.1037/ a0021042

Nguyen, T. L., Tian, Y. H., You, I. J., Lee, S. Y., and Jang, C. G. (2011). Modafinilinduced conditioned place preference via dopaminergic system in mice. Synapse 65, 733-741. doi: 10.1002/syn.20892

Nisell, M., Nomikos, G. G., and Svensson, T. H. (1994). Systemic nicotine-induced dopamine release in the rat nucleus accumbens is regulated by nicotinic receptors in the ventral tegmental area. Synapse 16, 36-44. doi: 10.1002/syn. 890160105

Nishino, S., and Mignot, E. (1997). Pharmacological aspects of human and canine narcolepsy. Prog. Neurobiol. 52, 27-78. doi: 10.1016/s0301-0082(96)00070-6

Nishino, S., Mao, J., Sampathkumaran, R., Shelton, J., and Mignot, E. (1998). Increased dopaminergic transmission mediates the wake-promoting effects of CNS stimulants. Sleep Res Online 1, 49-61.

Nordahl, T. E., Salo, R., and Leamon, M. (2003). Neuropsychological effects of chronic methamphetamine use on neurotransmitters and cognition: a review. J. Neuropsychiatry Clin. Neurosci. 15, 317-325. doi: 10.1176/jnp.15.3.317

Nuijten, M., Blanken, P., Van Den Brink, W., and Hendriks, V. (2015). Modafinil in the treatment of crack-cocaine dependence in the Netherlands: results of an open-label randomised controlled feasibility trial. J. Psychopharmacol. 29, 678-687. doi: 10.1177/0269881115582151

Nuijten, M., Blanken, P., Van Den Brink, W., Goudriaan, A. E., and Hendriks, V. M. (2016). Impulsivity and attentional bias as predictors of modafinil treatment outcome for retention and drug use in crack-cocaine dependent patients: results of a randomised controlled trial. J. Psychopharmacol. 30, 616-626. doi: 10.1177/ 0269881116645268

Okunola-Bakare, O. M., Cao, J., Kopajtic, T., Katz, J. L., Loland, C. J., Shi, L., et al. (2014). Elucidation of structural elements for selectivity across monoamine transporters: novel 2-[(diphenylmethyl)sulfinyl]acetamide (modafinil) analogues. J. Med. Chem. 57, 1000-1013. doi: 10.1021/jm401754x

Ozturk, A., and Deveci, E. (2014). Drug abuse of modafinil by a cannabis user. Bull. Clin. Psychopharmacol. 24, 405-407. doi: 10.5455/bcp.20130624013303

Paterson, N. E., Fedolak, A., Olivier, B., Hanania, T., Ghavami, A., and Caldarone, B. (2010). Psychostimulant-like discriminative stimulus and locomotor sensitization properties of the wake-promoting agent modafinil in rodents. Pharmacol. Biochem. Behav. 95, 449-456. doi: 10.1016/j.pbb.2010.03.006

Peacock, A., Leung, J., Larney, S., Colledge, S., Hickman, M., Rehm, J., et al. (2018). Global statistics on alcohol, tobacco and illicit drug use: 2017 status report. Addiction 113, 1905-1926. doi: 10.1111/add.14234

Peñaloza, R. A., Sarkar, U., Claman, D. M., and Omachi, T. A. (2013). Trends in on-label and off-label modafinil use in a nationally representative sample. JAMA Internal Med. 173, 704-706. doi: 10.1001/jamainternmed.2013.2807

Pettit, H. O., and Justice, J. B. (1989). Dopamine in the nucleus accumbens during cocaine self-administration as studied by in vivo microdialysis. Pharmacol. Biochem. Behav. 34, 899-904. doi: 10.1016/0091-3057(89)90291-8

Phillips, K. A., Epstein, D. H., and Preston, K. L. (2014). Psychostimulant addiction treatment. Neuropharmacology 87, 150-160. doi: 10.1016/j.neuropharm.2014. 04.002

Picciotto, M. R., Zoli, M., Rimondini, R., Léna, C., Marubio, L. M., Pich, E. M., et al. (1998). Acetylcholine receptors containing the $\beta 2$ subunit are involved in the reinforcing properties of nicotine. Nature 391, 173-177. doi: 10.1038/34413
Piérard, C., Liscia, P., Valleau, M., Drouet, I., Chauveau, F., Huart, B., et al. (2006). Modafinil-induced modulation of working memory and plasma corticosterone in chronically-stressed mice. Pharmacol. Biochem. Behav. 83, 1-8. doi: 10.1016/ j.pbb.2005.11.018

Pigeau, R., Naitoh, P., Buguet, A., Mccann, C., Baranski, J., Taylor, M., et al. (1995). Modafinil, d-amphetamine and placebo during 64 hours of sustained mental work. I. effects on mood, fatigue, cognitive performance and body temperature. J. Sleep Res. 4, 212-228. doi: 10.1111/j.1365-2869.1995.tb00172.x

Pontieri, F. E., Tanda, G., and Di Chiara, G. (1995). Intravenous cocaine, morphine, and amphetamine preferentially increase extracellular dopamine in the "shell" as compared with the "core" of the rat nucleus accumbens. Proc. Natl. Acad. Sci. U.S.A. 92, 12304-12308. doi: 10.1073/pnas.92.26.12304

Pontieri, F. E., Tanda, G., Orzi, F., and Di Chiara, G. (1996). Effects of nicotine on the nucleus accumbens and similarity to those of addictive drugs. Nature 382, 255-257. doi: 10.1038/382255a0

Porter, J. H., Prus, A. J., and Overton, D. A. (2018). Drug discrimination: historical origins, important concepts, and principles. Curr. Top. Behav. Neurosci. 39, 3-26. doi: 10.1007/7854_2018_40

Quisenberry, A. J., Prisinzano, T. E., and Baker, L. E. (2013). Modafinil alone and in combination with low dose amphetamine does not establish conditioned place preference in male sprague-dawley rats. Exp. Clin. Psychopharmacol. 21, 252-258. doi: 10.1037/a0031832

Raineri, M., Gonzalez, B., Goitia, B., Garcia-Rill, E., Krasnova, I. N., Cadet, J. L., et al. (2012). Modafinil abrogates methamphetamine-induced neuroinflammation and apoptotic effects in the mouse striatum. PLoS One 7:e46599. doi: 10.1371/journal.pone.0046599

Rao, Y., Liu, Z. W., Borok, E., Rabenstein, R. L., Shanabrough, M., Lu, M., et al. (2007). Prolonged wakefulness induces experience-dependent synaptic plasticity in mouse hypocretin/orexin neurons. J. Clin. Invest. 117, 4022-4033. doi: $10.1172 /$ jci32829

Redrobe, J. P., Bull, S., and Plath, N. (2010). Translational aspects of the novel object recognition task in rats abstinent following sub-chronic treatment with phencyclidine (PCP): effects of modafinil and relevance to cognitive deficits in schizophrenia. Front. Psychiatry 1:146.

Reichel, C. M., and See, R. E. (2010). Modafinil effects on reinstatement of methamphetamine seeking in a rat model of relapse. Psychopharmacology 210, 337-346. doi: 10.1007/s00213-010-1828-5

Reichel, C. M., and See, R. E. (2012). Chronic modafinil effects on drugseeking following methamphetamine self-administration in rats. Int. J. Neuropsychopharmacol. 15, 919-929. doi: 10.1017/s1461145711000988

Reichel, C. M., Gilstrap, M. G., Ramsey, L. A., and See, R. E. (2014). Modafinil restores methamphetamine induced object-in-place memory deficits in rats independent of glutamate $\mathrm{N}$-methyl-d-aspartate receptor expression. Drug Alcohol Depend. 134, 115-122. doi: 10.1016/j.drugalcdep.2013 .09 .018

Revel, F., Moreau, J., Pouzet, B., Mory, R., Bradaia, A., Buchy, D., et al. (2013). A new perspective for schizophrenia: TAAR1 agonists reveal antipsychotic-and antidepressant-like activity, improve cognition and control body weight. Mol. Psychiatry 18, 543-556. doi: 10.1038/mp.2012.57

Robertson, P., and Hellriegel, E. T. (2003). Clinical pharmacokinetic profile of modafinil. Clin. Pharmacokinet. 42, 123-137. doi: 10.2165/00003088200342020-00002

Robertson, P., Decory, H. H., Madan, A., and Parkinson, A. (2000). In vitro inhibition and induction of human hepatic cytochrome P450 enzymes by modafinil. Drug Metab. Dispos. 28, 664-671.

Robinson, T. E., and Kolb, B. (2004). Structural plasticity associated with exposure to drugs of abuse. Neuropharmacology 47, 33-46. doi: 10.1016/j.neuropharm. 2004.06.025

Rogers, J., De Santis, S., and See, R. (2008). Extended methamphetamine selfadministration enhances reinstatement of drug seeking and impairs novel object recognition in rats. Psychopharmacology 199, 615-624. doi: 10.1007/ s00213-008-1187-7

Rounsaville, B. J., Anton, S. F., Carroll, K., Budde, D., Prusoff, B. A., and Gawin, F. (1991). Psychiatric diagnoses of treatment-seeking cocaine abusers. Arch. Gen. Psychiatry 48, 43-51. doi: 10.1001/archpsyc.1991.01810250045005

Rowley, H. L., Kulkarni, R. S., Gosden, J., Brammer, R. J., Hackett, D., and Heal, D. J. (2014). Differences in the neurochemical and behavioural profiles of lisdexamfetamine methylphenidate and modafinil revealed by simultaneous 
dual-probe microdialysis and locomotor activity measurements in freelymoving rats. J. Psychopharmacol. 28, 254-269. doi: 10.1177/0269881113513850

Rush, C. R., Kelly, T. H., Hays, L. R., Baker, R., and Wooten, A. (2002a). Acute behavioral and physiological effects of modafinil in drug abusers. Behav. Pharmacol. 13, 105-115. doi: 10.1097/00008877-200203000-00002

Rush, C. R., Kelly, T. H., Hays, L. R., and Wooten, A. F. (2002b). Discriminativestimulus effects of modafinil in cocaine-trained humans. Drug Alcohol Depend. $67,311-322$.

Russo, S. J., Mazei-Robison, M. S., Ables, J. L., and Nestler, E. J. (2009). Neurotrophic factors and structural plasticity in addiction. Neuropharmacology $56,73-82$.

Sahakian, B. J., and Morein-Zamir, S. (2011). Neuroethical issues in cognitive enhancement. J. Psychopharmacol. 25, 197-204. doi: 10.1177/ 0269881109106926

Sahakian, B., Robbins, T., Morgan, M., and Iversen, S. (1975). The effects of psychomotor stimulants on stereotypy and locomotor activity in sociallydeprived and control rats. Brain Res. 84, 195-205. doi: 10.1016/0006-8993(75) 90975-0

Sakurai, T. (2007). The neural circuit of orexin (hypocretin): maintaining sleep and wakefulness. Nat. Rev. Neurosci. 8, 171-181. doi: 10.1038/nrn2092

Salamone, J. D., Correa, M., Mingote, S., and Weber, S. M. (2003). Nucleus accumbens dopamine and the regulation of effort in food-seeking behavior: implications for studies of natural motivation, psychiatry, and drug abuse. J. Pharmacol. Exp. Ther. 305, 1-8. doi: 10.1124/jpet.102.035063

Salo, R., Flower, K., Kielstein, A., Leamon, M. H., Nordahl, T. E., and Galloway, G. P. (2011). Psychiatric comorbidity in methamphetamine dependence. Psychiatry Res. 186, 356-361. doi: 10.1016/j.psychres.2010.09.014

SAMHSA (2018). Mental Health Indicators in the United States: Results from the 2018 National Survey on Drug Use and Health. Rockville, MD: SAMHSA.

Sandoval, V., Riddle, E. L., Ugarte, Y. V., Hanson, G. R., and Fleckenstein, A. E. (2001). Methamphetamine-induced rapid and reversible changes in dopamine transporter function: an in vitro model. J. Neurosci. 21, 1413-1419. doi: 10. 1523/jneurosci.21-04-01413.2001

Sarnyai, Z., Bíró, É, Gardi, J., Vecsernyés, M., Julesz, J., and Telegdy, G. (1995). Brain corticotropin-releasing factor mediates 'anxiety-like'behavior induced by cocaine withdrawal in rats. Brain Res. 675, 89-97. doi: 10.1016/0006-8993(95) 00043-p

Saunders, C., Ferrer, J. V., Shi, L., Chen, J., Merrill, G., Lamb, M. E., et al. (2000). Amphetamine-induced loss of human dopamine transporter activity: an internalization-dependent and cocaine-sensitive mechanism. Proc. Natl. Acad. Sci. U.S.A. 97, 6850-6855. doi: 10.1073/pnas.110035297

Sawaguchi, T., and Goldman-Rakic, P. S. (1991). D1 dopamine receptors in prefrontal cortex: involvement in working memory. Science 251, 947-950. doi: 10.1126/science. 1825731

Sawaguchi, T., and Goldman-Rakic, P. S. (1994). The role of D1-dopamine receptor in working memory: local injections of dopamine antagonists into the prefrontal cortex of rhesus monkeys performing an oculomotor delayedresponse task. J. Neurophysiol. 71, 515-528. doi: 10.1152/jn.1994.71.2.515

Scammell, T. E., Estabrooke, I. V., Mccarthy, M. T., Chemelli, R. M., Yanagisawa, M., Miller, M. S., et al. (2000). Hypothalamic arousal regions are activated during modafinil-induced wakefulness. J. Neurosci. 20, 8620-8628. doi: 10. 1523/jneurosci.20-22-08620.2000

Schierenbeck, T., Riemann, D., Berger, M., and Hornyak, M. (2008). Effect of illicit recreational drugs upon sleep: cocaine, ecstasy and marijuana. Sleep Med. Rev. 12, 381-389. doi: 10.1016/j.smrv.2007.12.004

Schmaal, L., Goudriaan, A., Joos, L., Dom, G., Pattij, T., Van Den Brink, W., et al. (2014). Neural substrates of impulsive decision making modulated by modafinil in alcohol-dependent patients. Psychol. Med. 44, 2787-2798. doi: $10.1017 / \mathrm{s} 0033291714000312$

Schmaal, L., Joos, L., Koeleman, M., Veltman, D. J., Van Den Brink, W., and Goudriaan, A. E. (2013). Effects of modafinil on neural correlates of response inhibition in alcohol-dependent patients. Biol. Psychiatry 73, 211-218. doi: 10.1016/j.biopsych.2012.06.032

Schmitt, K. C., and Reith, M. E. (2011). The atypical stimulant and nootropic modafinil interacts with the dopamine transporter in a different manner than classical cocaine-like inhibitors. PLoS One 6:225790. doi: 10.1371/journal.pone. 0025790
Schmitz, J. M., Green, C. E., Stotts, A. L., Lindsay, J. A., Rathnayaka, N. S., Grabowski, J., et al. (2014). A two-phased screening paradigm for evaluating candidate medications for cocaine cessation or relapse prevention: Modafinil, Levodopa-Carbidopa, Naltrexone. Drug Alcohol Depend. 136, 100-107. doi: 10.1016/j.drugalcdep.2013.12.015

Schnoll, R. A., Wileyto, E. P., Pinto, A., Leone, F., Gariti, P., Siegel, S., et al. (2008). A placebo-controlled trial of modafinil for nicotine dependence. Drug Alcohol Depend. 98, 86-93. doi: 10.1016/j.drugalcdep.2008.04.008

Schork, N. J. (2015). Personalized medicine: time for one-person trials. Nature 520, 609-611. doi: 10.1038/520609a

Schwartz, J. R. (2005). Modafinil: new indications for wake promotion. Expert Opin. Pharmacother. 6, 115-129. doi: 10.1517/14656566.6.1.115

Schwartz, M. D., Palmerston, J. B., Lee, D. L., Hoener, M. C., and Kilduff, T. S. (2018). Deletion of trace amine-associated receptor 1 attenuates behavioral responses to caffeine. Front. Pharmacol. 9:35.

Shanmugasundaram, B., Aher, Y. D., Aradska, J., Ilic, M., Daba Feyissa, D., Kalaba, P., et al. (2017). R-Modafinil exerts weak effects on spatial memory acquisition and dentate gyrus synaptic plasticity. PLoS One 12:e0179675. doi: 10.1371/ journal.pone.0179675

Sharif, S., Guirguis, A., Fergus, S., and Schifano, F. (2021). The use and impact of cognitive enhancers among university students: a systematic review. Brain Sci. 11:355. doi: 10.3390/brainsci1 1030355

Shearer, J., Darke, S., Rodgers, C., Slade, T., Van Beek, I., Lewis, J., et al. (2009). A double-blind, placebo-controlled trial of modafinil (200 $\mathrm{mg} /$ day) for methamphetamine dependence. Addiction 104, 224-233. doi: 10.1111/j.13600443.2008.02437.x

Shuman, T., Cai, D. J., Sage, J. R., and Anagnostaras, S. G. (2012). Interactions between modafinil and cocaine during the induction of conditioned place preference and locomotor sensitization in mice: implications for addiction. Behav. Brain Res. 235, 105-112. doi: 10.1016/j.bbr.2012.07.039

Shuman, T., Wood, S. C., and Anagnostaras, S. G. (2009). Modafinil and memory: effects of modafinil on Morris water maze learning and Pavlovian fear conditioning. Behav. Neurosci. 123, 257-266. doi: 10.1037/a0014366

Slack, R. D., Ku, T. C., Cao, J., Giancola, J. B., Bonifazi, A., Loland, C. J., et al. (2020). Structure-activity relationships for a series of (Bis(4fluorophenyl)methyl)sulfinyl Alkyl Alicyclic Amines at the dopamine transporter: functionalizing the terminal Nitrogen affects affinity, selectivity, and metabolic stability. J. Med. Chem. 63, 2343-2357. doi: 10.1021/acs.jmedchem.9b01188

Sofuoglu, M., Devito, E. E., Waters, A. J., and Carroll, K. M. (2013). Cognitive enhancement as a treatment for drug addictions. Neuropharmacology 64, 452463. doi: 10.1016/j.neuropharm.2012.06.021

Sofuoglu, M., Devito, E. E., Waters, A. J., and Carroll, K. M. (2016). Cognitive function as a transdiagnostic treatment target in stimulant use disorders. J. Dual Diagnosis 12, 90-106. doi: 10.1080/15504263.2016.1146383

Spencer, T. J., Madras, B. K., Bonab, A. A., Dougherty, D. D., Clarke, A., Mirto, T., et al. (2010). A positron emission tomography study examining the dopaminergic activity of armodafinil in adults using [11C] altropane and [11C] raclopride. Biol. Psychiatry 68, 964-970. doi: 10.1016/j.biopsych.2010.08.026

Stoops, W. W., Lile, J. A., Fillmore, M. T., Glaser, P. E., and Rush, C. R. (2005). Reinforcing effects of modafinil: influence of dose and behavioral demands following drug administration. Psychopharmacology 182, 186-193. doi: 10. 1007/s00213-005-0044-1

Sulzer, D., Sonders, M. S., Poulsen, N. W., and Galli, A. (2005). Mechanisms of neurotransmitter release by amphetamines: a review. Prog. Neurobiol. 75, 406-433. doi: 10.1016/j.pneurobio.2005.04.003

Taber, K. H., Black, D. N., Porrino, L. J., and Hurley, R. A. (2012). Neuroanatomy of dopamine: reward and addiction. J. Neuropsychiatry Clin. Neurosci. 24, 1-4. doi: 10.1176/appi.neuropsych.24.1.1

Tahsili-Fahadan, P., Carr, G. V., Harris, G. C., and Aston-Jones, G. (2010). Modafinil blocks reinstatement of extinguished opiate-seeking in rats: mediation by a glutamate mechanism. Neuropsychopharmacology 35, 22032210. doi: $10.1038 /$ npp.2010.94

Tanda, G., Hersey, M., Hempel, B., Xi, Z.-X., and Newman, A. H. (2021). Modafinil and its structural analogs as atypical dopamine uptake inhibitors and potential medications for psychostimulant use disorder. Curr. Opin. Pharmacol. 56, 13-21. doi: 10.1016/j.coph.2020.07.007 
Tanda, G., Newman, A. H., Ebbs, A. L., Tronci, V., Green, J. L., Tallarida, R. J., et al. (2009). Combinations of cocaine with other dopamine uptake inhibitors: assessment of additivity. J. Pharmacol. Exp. Ther. 330, 802-809. doi: 10.1124/ jpet.109.154302

Tanda, G., Pontieri, F. E., and Di Chiara, G. (1997a). Cannabinoid and heroin activation of mesolimbic dopamine transmission by a common mul opioid receptor mechanism. Science 276, 2048-2050. doi: 10.1126/science.276.5321. 2048

Tanda, G., Pontieri, F. E., Frau, R., and Di Chiara, G. (1997b). Contribution of blockade of the noradrenaline carrier to the increase of extracellular dopamine in the rat prefrontal cortex by amphetamine and cocaine. Eur. J. Neurosci. 9, 2077-2085. doi: 10.1111/j.1460-9568.1997.tb01375.x

Thomas, M., Kalivas, P., and Shaham, Y. (2008). Neuroplasticity in the mesolimbic dopamine system and cocaine addiction. Br. J. Pharmacol. 154, 327-342. doi: 10.1038/bjp.2008.77

Torrens, M., and Rossi, P. (2015). "Mood disorders and addiction," in Cooccurring Addictive and Psychiatric Disorders, eds F. Moggi and G. Dom (Berlin: Springer), 103-117. doi: 10.1007/978-3-642-45375-5_8

Touret, M., Sallanon-Moulin, M., Fages, C., Roudier, V., Didier-Bazes, M., Roussel, B., et al. (1994). Effects of modafinil-induced wakefulness on glutamine synthetase regulation in the rat brain. Mol. Brain Res. 26, 123-128. doi: 10 . 1016/0169-328x(94)90082-5

Trulson, M. E. (1985). Simultaneous recording of substantia nigra neurons and voltammetric release of dopamine in the caudate of behaving cats. Brain Res. Bull. 15, 221-223. doi: 10.1016/0361-9230(85)90140-6

Tseng, K. Y., and O’Donnell, P. (2004). Dopamine-glutamate interactions controlling prefrontal cortical pyramidal cell excitability involve multiple signaling mechanisms. J. Neurosci. 24, 5131-5139. doi: 10.1523/jneurosci.102104.2004

Tseng, K. Y., and O'Donnell, P. (2007). D2 dopamine receptors recruit a GABA component for their attenuation of excitatory synaptic transmission in the adult rat prefrontal cortex. Synapse 61, 843-850. doi: 10.1002/syn.20432

Tunstall, B. J., Ho, C. P., Cao, J., Vendruscolo, J. C., Schmeichel, B. E., Slack, R. D., et al. (2018). Atypical dopamine transporter inhibitors attenuate compulsivelike methamphetamine self-administration in rats. Neuropharmacology 131, 96-103. doi: 10.1016/j.neuropharm.2017.12.006

Turner, C., Belyavin, A., and Nicholson, A. (2014). Duration of activity and mode of action of modafinil: Studies on sleep and wakefulness in humans. J. Psychopharmacol. 28, 643-654. doi: 10.1177/0269881113508173

Turner, D. C., Robbins, T. W., Clark, L., Aron, A. R., Dowson, J., and Sahakian, B. J. (2003). Cognitive enhancing effects of modafinil in healthy volunteers. Psychopharmacology 165, 260-269. doi: 10.1007/s00213-002-1250-8

Tzschentke, T. M. (2007). Review on CPP: measuring reward with the conditioned place preference (CPP) paradigm: update of the last decade. Addict. Biol. 12, 227-462. doi: 10.1111/j.1369-1600.2007.00070.x

Urbano, F. J., Leznik, E., and Llinás, R. R. (2007). Modafinil enhances thalamocortical activity by increasing neuronal electrotonic coupling. Proc. Natl. Acad. Sci. U.S.A. 104, 12554-12559. doi: 10.1073/pnas.0705087104

US Modafinil in Narcolepsy Multicenter Study Group (1998). Randomized trial of modafinil for the treatment of pathological somnolence in narcolepsy. Ann. Neurol. 43, 88-97. doi: 10.1002/ana.410430115

US Modafinil in Narcolepsy Multicenter Study Group (2000). Randomized trial of modafinil as a treatment for the excessive daytime somnolence of narcolepsy. Neurology 54, 1166-1175. doi: 10.1212/wnl.54.5.1166

Valentino, R. J., and Volkow, N. D. (2020). Drugs, sleep, and the addicted brain. Neuropsychopharmacology 45, 3-5. doi: 10.1038/s41386-019-0465-x

Velázquez-Sánchez, C., Santos, J. W., Smith, K. L., Ferragud, A., Sabino, V., and Cottone, P. (2015). Seeking behavior, place conditioning, and resistance to conditioned suppression of feeding in rats intermittently exposed to palatable food. Behav. Neurosci. 129, 219-224. doi: 10.1037/bne0000042

Verma, V. (2015). Classic studies on the interaction of cocaine and the dopamine transporter. Clin. Psychopharmacol. Neurosci. 13, 227-238. doi: 10.9758/cpn. 2015.13.3.227

Verrico, C. D., Haile, C. N., Mahoney Iii, J. J., Thompson-Lake, D. G., Newton, T. F., De La Garza, et al. (2014). Treatment with modafinil and escitalopram, alone and in combination, on cocaine-induced effects: a randomized, double blind, placebo-controlled human laboratory study. Drug Alcohol Depend. 141, 72-78. doi: 10.1016/j.drugalcdep.2014.05.008
Vocci, F. J., and Montoya, I. D. (2009). Psychological treatments for stimulant misuse, comparing and contrasting those for amphetamine dependence and those for cocaine dependence. Curr. Opin. Psychiatry 22, 263-268. doi: 10.1097/ yco.0b013e32832a3b44

Volkow, N. D., Fowler, J. S., Logan, J., Alexoff, D., Zhu, W., Telang, F., et al. (2009). Effects of modafinil on dopamine and dopamine transporters in the male human brain: clinical implications. JAMA 301, 1148-1154. doi: 10.1001/ jama.2009.351

Volkow, N. D., Wang, G.-J., Fowler, J. S., Tomasi, D., and Telang, F. (2011). Addiction: beyond dopamine reward circuitry. Proc. Natl. Acad. Sci. U.S.A. 108, 15037-15042. doi: 10.1073/pnas.1010654108

Vosburg, S. K., Hart, C. L., Haney, M., Rubin, E., and Foltin, R. W. (2010). Modafinil does not serve as a reinforcer in cocaine abusers. Drug Alcohol Depend. 106, 233-236. doi: 10.1016/j.drugalcdep.2009.09.002

Wang, X.-F., Bi, G.-H., He, Y., Yang, H.-J., Gao, J.-T., Okunola-Bakare, O. M., et al. (2015). R-modafinil attenuates nicotine-taking and nicotine-seeking behavior in alcohol-preferring rats. Neuropsychopharmacology 40, 1762-1771. doi: 10 . 1038/npp.2015.24

Ward, C. P., Harsh, J. R., York, K. M., Stewart, K. L., and Mccoy, J. G. (2004). Modafinil facilitates performance on a delayed nonmatching to position swim task in rats. Pharmacol. Biochem. Behav. 78, 735-741. doi: 10.1016/j.pbb.2004. 05.005

Warot, D., Corruble, E., Payan, C., Weil, J., and Puech, A. (1993). Subjective effects of modafinil, a new central adrenergic stimulant in healthy volunteers: a comparison with amphetamine, caffeine and placebo. Eur. Psychiatry 8, 201-208. doi: 10.1017/s0924933800002923

Waters, K. A., Burnham, K. E., O'connor, D., Dawson, G. R., and Dias, R. J. J. O. P. (2005). Assessment of modafinil on attentional processes in a five-choice serial reaction time test in the rat. J. Psychopharmacol. 19, 149-158. doi: 10.1177/ 0269881105048995

Willie, J., Renthal, W., Chemelli, R., Miller, M., Scammell, T., Yanagisawa, M., et al. (2005). Modafinil more effectively induces wakefulness in orexin-null mice than in wild-type littermates. Neuroscience 130, 983-995. doi: 10.1016/ j.neuroscience.2004.10.005

Wise, R. A. (2008). Dopamine and reward: the anhedonia hypothesis 30 years on. Neurotox. Res. 14, 169-183. doi: 10.1007/bf03033808

Wise, R. A., and Bozarth, M. A. (1987). A psychomotor stimulant theory of addiction. Psychol. Rev. 94, 469-492. doi: 10.1037/0033-295x. 94.4.469

Wise, R. A., and Robble, M. A. (2020). Dopamine and Addiction. Annu. Rev. Psychol. 71, 79-106.

Wise, R. A., and Rompre, P.-P. (1989). Brain dopamine and reward. Annu. Rev. Psychol. 40, 191-225.

Wisor, J. P., Dement, W. C., Aimone, L., Williams, M., and Bozyczko-Coyne, D. (2006). Armodafinil, the R-enantiomer of modafinil: wake-promoting effects and pharmacokinetic profile in the rat. Pharmacol. Biochem. Behav. 85, 492499. doi: 10.1016/j.pbb.2006.09.018

Wisor, J. P., Nishino, S., Sora, I., Uhl, G. H., Mignot, E., and Edgar, D. M. (2001). Dopaminergic role in stimulant-induced wakefulness. J. Neurosci. 21, 1787-1794. doi: 10.1523/jneurosci.21-05-01787.2001

Wong, Y. N., Simcoe, D., Hartman, L. N., Laughton, W. B., King, S. P., Mccormick, G. C., et al. (1999). A double-blind, placebo-controlled, ascendingdose evaluation of the pharmacokinetics and tolerability of modafinil tablets in healthy male volunteers. J. Clin. Pharmacol. 39, 30-40. doi: 10.1177/ 00912709922007534

Woolverton, W. L., and Johnson, K. M. (1992). Neurobiology of cocaine abuse. Trends Pharmacol. Sci. 13, 193-200. doi: 10.1016/0165-6147(92)90063-c

Wuo-Silva, R., Fukushiro, D. F., Borçoi, A. R., Fernandes, H. A., Procópio-Souza, R., Hollais, A. W., et al. (2011). Addictive potential of modafinil and crosssensitization with cocaine: a pre-clinical study. Addict. Biol. 16, 565-579. doi: 10.1111/j.1369-1600.2011.00341.x

Wuo-Silva, R., Fukushiro, D. F., Hollais, A. W., Santos-Baldaia, R., MáriKawamoto, E., Berro, L. F., et al. (2016). Modafinil induces rapidonset behavioral sensitization and cross-sensitization with cocaine in mice: implications for the addictive potential of modafinil. Front. Pharmacol. 7:420.

Wuo-Silva, R., Fukushiro-Lopes, D. F., Fialho, B. P., Hollais, A. W., Santos-Baldaia, R., Marinho, E. A., et al. (2019). Participation of dopamine D1 and D2 receptors 
in the rapid-onset behavioral sensitization to modafinil. Front. Pharmacol. $10: 211$.

Xie, Z., and Miller, G. M. (2009). A receptor mechanism for methamphetamine action in dopamine transporter regulation in brain. J. Pharmacol. Exp. Ther. 330, 316-325. doi: 10.1124/jpet.109.153775

Yan, W.-W., Yao, L.-H., Chen, C., Wang, H.-X., Li, C.-H., Huang, J.-N., et al. (2015). Effects of modafinil on behavioral learning and hippocampal synaptic transmission in rats. Int. Neurourol. J. 19, 220-227. doi: 10.5213/inj.2015.19.4. 220

Young, J. W., and Geyer, M. A. (2010). Action of modafinil-increased motivation via the dopamine transporter inhibition and D1 receptors? Biol. Psychiatry 67, 784-787. doi: 10.1016/j.biopsych.2009.12.015

Young, J. W., Kooistra, K., and Geyer, M. A. (2011). Dopamine receptor mediation of the exploratory/hyperactivity effects of modafinil. Neuropsychopharmacology 36, 1385-1396. doi: 10.1038/npp.2011.23

Yu, X., Ma, Y., Harding, E. C., Yustos, R., Vyssotski, A. L., Franks, N. P., et al. (2019). Genetic lesioning of histamine neurons increases sleep-wake fragmentation and reveals their contribution to modafinil-induced wakefulness. Sleep 42:zsz031.

Zager, A., Brandão, W. N., Margatho, R. O., Peron, J. P., Tufik, S., Andersen, M. L., et al. (2018). The wake-promoting drug Modafinil prevents motor impairment in sickness behavior induced by LPS in mice: role for dopaminergic D1 receptor. Prog. Neuro Psychopharmacol. Biol. Psychiatry 81, 468-476. doi: 10.1016/j. pnpbp.2017.05.003

Zahniser, N. R., and Sorkin, A. (2004). Rapid regulation of the dopamine transporter: role in stimulant addiction? Neuropharmacology 47, 80-91. doi: 10.1016/j.neuropharm.2004.07.010

Zahniser, N. R., and Sorkin, A. (2009). Trafficking of dopamine transporters in psychostimulant actions. Semin. Cell Dev. Biol. 20, 411-417.
Zhang, H.-Y., Bi, G.-H., Yang, H.-J., He, Y., Xue, G., Cao, J., et al. (2017). The novel modafinil analog, JJC8-016, as a potential cocaine abuse pharmacotherapeutic. Neuropsychopharmacology 42, 1871-1883. doi: 10.1038/npp.2017.41

Zhang, L., Looney, D., Taub, D., Chang, S. L., Way, D., Witte, M. H., et al. (1998). Cocaine opens the blood-brain barrier to HIV-1 invasion. J. Neurovirol. 4, 619-626. doi: 10.3109/13550289809114228

Zhu, J., and Reith, M. (2008). Role of the dopamine transporter in the action of psychostimulants, nicotine, and other drugs of abuse. CNS Neurol. Disord. Drug Targets 7, 393-409. doi: 10.2174/1871527087869 27877

Zolkowska, D., Jain, R., Rothman, R. B., Partilla, J. S., Roth, B. L., Setola, V., et al. (2009). Evidence for the involvement of dopamine transporters in behavioral stimulant effects of modafinil. J. Pharmacol. Exp. Ther. 329, 738-746. doi: $10.1124 /$ jpet.108.146142

Conflict of Interest: The authors declare that the research was conducted in the absence of any commercial or financial relationships that could be construed as a potential conflict of interest.

The reviewer TK declared a past co-authorship with one of the authors, AN, to the handling editor.

Copyright (c) 2021 Hersey, Bacon, Bailey, Coggiano, Newman, Leggio and Tanda. This is an open-access article distributed under the terms of the Creative Commons Attribution License (CC BY). The use, distribution or reproduction in other forums is permitted, provided the original author(s) and the copyright owner(s) are credited and that the original publication in this journal is cited, in accordance with accepted academic practice. No use, distribution or reproduction is permitted which does not comply with these terms. 